\title{
Interactions between TULP3 tubby domain cargo site and ARL13B amphipathic helix promote lipidated protein transport to cilia
}

Vivek Reddy Palicharla ${ }^{1}$, Sun-Hee Hwang ${ }^{1}$, Bandarigoda N. Somatilaka ${ }^{1,2}$, Hemant B. Badgandi $^{1,3}$, Emilie Legué ${ }^{4}$, Vanna M. Tran ${ }^{1,5}$, Jeffrey B. Woodruff ${ }^{1}$, Karel F. Liem Jr. ${ }^{4}$, and Saikat Mukhopadhyay ${ }^{1^{*}}$.

${ }^{1}$ Department of Cell Biology, University of Texas Southwestern Medical Center, Dallas, TX, 75390.

2Present address: Department of Dermatology, University of Texas Southwestern Medical Center, Dallas, TX, 75390.

${ }^{3}$ Present address: Department of Chemistry, Vanderbilt University, Nashville, TN, 37235.

${ }^{4}$ Vertebrate Developmental Biology Program, Department of Pediatrics, Yale University

School of Medicine, New Haven, CT, 06520.

${ }^{5}$ Present address: Tetrad Graduate Program, University of California, San Francisco, San Francisco, CA, 94158.

${ }^{*}$ Correspondence:

Email: Saikat.Mukhopadhyay@utsouthwestern.edu

Running title: TULP3 traffics ARL13B and lipidated proteins to cilia. 


\section{Abstract}

The tubby family protein-TULP3 coordinates with the intraflagellar transport complex-A (IFT-A) in trafficking certain transmembrane proteins to cilia. These transmembrane cargoes have short motifs that are necessary and sufficient for TULP3-mediated trafficking. However, whether TULP3 regulates trafficking of membrane-associated proteins is not well understood. Here we show that TULP3 is required for transport of the atypical GTPase ARL13B into cilia, and for ciliary enrichment of ARL13B-dependent farnesylated and myristoylated proteins. ARL13B transport requires TULP3 binding to IFT-A core but not to phosphoinositides, unlike transmembrane cargo transport that requires binding to both by TULP3. A conserved lysine in TULP3's tubby domain mediates direct ARL13B binding and trafficking of lipidated and transmembrane cargoes. An Nterminal amphipathic helix in ARL13B flanking the palmitoylation site mediates binding to TULP3 and directs trafficking to cilia even in absence of palmitoylation and RVxP sorting motif. Therefore, TULP3 transports transmembrane proteins and ARL13B into cilia by capture of short sequences through a shared tubby domain site.

Keywords: Cilia; Tubby; Tulp3; intraflagellar transport; Arl13b; GTPase; phosphoinositide; lipidation; trafficking; cyst. 


\section{Introduction}

The primary cilium is a microtubule based dynamic cellular appendage that is templated from the mother centriole of the centrosome-the basal body (Anvarian et al., 2019). Cilia are present in multiple cell types and organs and can transduce cellular response to extracellular signals. These signals include hedgehog morphogens that regulate multiple developmental and regenerative programs (Kopinke et al., 2020). Signaling outputs from cilia also maintain renal tubular homeostasis preventing cystogenesis (Ma, 2021). The ciliary membrane has a distinct protein and lipid composition despite being contiguous with the plasma membrane (Nachury and Mick, 2019). Multiple transmembrane (Hilgendorf et al., 2016) and lipidated proteins are preferentially associated with the ciliary membrane. The lipidated proteins include palmitoylated proteins such as ARL13B (Caspary et al., 2007; Duldulao et al., 2009), farnesylated proteins such as INPP5E (Bielas et al., 2009) and myristoylated proteins such as NPHP3 (Shiba et al., 2010). Deciphering mechanisms underlying ciliary membrane compartmentalization is necessary to understand how signaling at and by cilia contributes to morpho-phenotypic outcomes.

ARL13B is an atypical GTPase that is highly enriched in the primary cilium. Mutations in $A R L 13 B$ in humans are associated with classic Joubert syndrome, a ciliopathy associated with cerebellar vermis hypoplasia, hypotonia, intellectual disability (Cantagrel et al., 2008) and occasionally accompanied with renal cysts, retinal impairment and obesity (Thomas et al., 2015). ARL13B acts as a GEF for the GTPase ARL3, converting inactive ARL3-GDP to active ARL3GTP (Gotthardt et al., 2015; Ivanova et al., 2017). ARL3-GTP promotes ciliary release of multiple lipidated cargoes including farnesylated and myristoylated proteins from their respective carrier proteins-Pde6 $\delta$ (Humbert et al., 2012) and Unc119b (Wright et al., 2011). Given that ARL13B potentially regulates levels of multiple lipidated proteins inside cilia, it seems logical that it affects ciliary signaling. However, the role of trafficking of ARL13B into cilia is tissue specific. The zebrafish arl13b (scorpion) allele has nephric duct dilatation phenotypes, and analysis of 
phenotypic rescue using arl13b variants in this model suggest that ciliary localization is essential for in vivo function of Arl13b (Duldulao et al., 2009; Sun et al., 2004). However, a mouse knockin at the endogenous locus, which prevents the mutant protein from localizing to cilia $\left(A r / 13 b^{V 358 A}\right)$, is not affected in neural tube patterning (Gigante et al., 2020), unlike the null Arl13b $b^{\text {hnn }}$ mutant where neural tube patterning is affected (Caspary et al., 2007). A precise understanding of mechanisms trafficking ARL13B to cilia could perhaps shed light on the differences observed in the role of ciliary trafficking of ARL13B in different contexts.

A definitive ciliary targeting mechanism for ARL13B has been lacking, mostly because of contrasting results with regards to ciliary targeting sequences that are necessary but not sufficient for trafficking (see supplemental text). One motif that has stood out as being necessary for ciliary localization is the RVxP motif in the C-terminus (Mariani et al., 2016). Other studies have reported cellular aggregates upon deletion of the RVxP motif (Cevik et al., 2013; Nozaki et al., 2017), along with proximal to distal ciliary mislocalization in C. elegans phasmid cilia (Cevik et al., 2013). The $\mathrm{RVxP}$ motif was initially discovered as a C-terminal motif involved in post-Golgi trafficking of Rhodopsin (Deretic et al., 2005; Wang et al., 2012) and other proteins in the secretory pathway, including the olfactory CNGB1B subunit (Jenkins et al., 2006), and Polycystin-1 and Polycystin-2 channels (Geng et al., 2006; Ward et al., 2011). ARL13B is a cytosolic protein that is palmitoylated, and the RVxP motif is not strictly at the end of the protein (unlike within 10 aa of the C-terminus in other secretory pathway proteins). The interpretations of experiments in different settings are further complicated by ciliary localization assays of ARL13B mutants performed in the wildtype (Cevik et al., 2010; Cevik et al., 2013; Hori et al., 2008; Nozaki et al., 2017) or null background (Duldulao et al., 2009; Larkins et al., 2011; Mariani et al., 2016). Experiments in $C$. elegans have also demonstrated dominant negative roles of mutants (Cevik et al., 2010; Cevik et al., 2013), suggesting mutual interactions in compromising wild-type protein function. Self-association of ARL13B through its $\mathrm{N}$-terminal domain has been reported (Hori et al., 2008) that could explain some of the above dominant negative effects, although others have 
reported lack of dimerization of the purified GTPase domain (Miertzschke et al., 2014). Some of the ARL13B mutants, such as deletion of the RVxP motif or lack of the coiled coil, form cellular aggregates and could be indirectly affecting ciliary targeting (Cevik et al., 2013; Nozaki et al., 2017). In sum, the mechanism of ARL13B trafficking by the RVxP motif and in different contexts has remained unclear.

The tubby family protein, TULP3, functions in coordination with the intraflagellar transport complex-A (IFT-A) to determine ciliary trafficking of transmembrane proteins without affecting their extraciliary pools or disrupting cilia (Mukhopadhyay et al., 2010). These cargoes include GPCRs and polycystin channels (Badgandi et al., 2017). We proposed three steps during TULP3 mediated trafficking to cilia that consist of 1) capturing of the membrane localized cargoes by TULP3's C-terminal tubby domain in a $\mathrm{PI}(4,5) \mathrm{P}_{2}$-dependent manner, 2) trafficking into the primary cilium via IFT-A core binding to the N-terminus of TULP3, and 3) release of GPCR cargoes from TULP3 into the PI(4,5) $\mathrm{P}_{2}$ deficient ciliary membrane. TULP3 and the related but brain-specific Tubby are now established as the major conduit for trafficking of multiple types of membrane cargoes to mammalian cilia (Barbeito et al., 2020; Chavez et al., 2015; Dateyama et al., 2019; Garcia-Gonzalo et al., 2015; Hilgendorf et al., 2019; Hirano et al., 2017; Loktev and Jackson, 2013; Mukhopadhyay et al., 2017; Sun et al., 2012; Wu et al., 2020).

In line with a role of TULP3 in trafficking multiple cargoes, TULP3 and IFT-A have been implicated in neural tube patterning (Legue and Liem, 2020; Liem et al., 2012; Mukhopadhyay et al., 2013; Norman et al., 2009; Qin et al., 2011), renal cystogenesis (Hwang et al., 2019; Legue and Liem, 2019; Wang et al., 2020) and adipogenesis (Hilgendorf et al., 2019; Wu et al., 2020). In some cases, such as neural tube development (Mukhopadhyay et al., 2013) or adipogenesis (Hilgendorf et al., 2019), the relevant TULP3-regulated cargoes are likely to be GPCRs, but for other tissues such as renal cystogenesis, the TULP3 cargoes have not been established. We recently showed that kidney collecting duct specific embryonic or juvenile-onset Tulp3 knockout (ko) in mice results in postnatal kidney cystogenesis, whereas adult-onset loss causes delayed 
cystogenesis (Hwang et al., 2019; Legue and Liem, 2019). Interestingly, we observed that ARL13B pools in cilia of Tulp3 conditional ko (cko) collecting ducts were depleted prior to cyst formation in juveniles and in adults, indicating a role for TULP3 in ARL13B ciliary localization (Hwang et al., 2019; Legue and Liem, 2019). However, the direct role of TULP3 in trafficking ARL13B to cilia and relevance of any such trafficking mechanisms have remained unknown.

Our earlier studies showed that for almost all TULP3 dependent transmembrane cargoes, the ciliary targeting motifs were short peptide sequences. When fused to membrane anchors, these sequences were in proximity to TULP3. These motifs were both necessary and sufficient for ciliary trafficking (Badgandi et al., 2017). Here, we show that TULP3 is required for ciliary trafficking of ARL13B, and enrichment of ARL13B-dependent farnesylated and myristoylated proteins in cilia. We identify an amphipathic helix in the N-terminus of ARL13B to mediate binding to TULP3 and in determining ciliary localization of ARL13B. We identify the corresponding site in the tubby domain of TULP3 that mediates ciliary trafficking of both ARL13B and transmembrane cargoes. Thus, TULP3, by binding to short sequences through shared tubby domain interfaces determines levels of transmembrane cargoes and ARL13B-dependent lipidated cargoes in cilia. 


\section{Results}

\section{TULP3 is required for ciliary trafficking of ARL13B}

We recently observed that cilia in kidney collecting ducts deleted for Tulp3 lacked Arl13b (Hwang et al., 2019; Legue and Liem, 2019). To further investigate the role of Tulp3 in ciliary trafficking of Arl13b in different contexts, we first tested Arl13b ciliary trafficking in mouse embryonic fibroblasts (MEFs) obtained from wild type (WT) and Tulp3 knockout (ko) mice (Legue and Liem, 2019). Arl13b was primarily localized to cilia in wild type (WT) MEFs whereas ciliary localization was abrogated in Tulp3 ko MEFs, suggesting that Arl13b ciliary localization is Tulp3 dependent

(Figure 1A, 1B). Ciliary localization of Gpr161, a known GPCR cargo for Tulp3 (Mukhopadhyay et al., 2013), was also lost in Tulp3 ko MEFs as expected (Figure 1B, S1A). Tulp3 ko MEFs had no observable defects in ciliation with respect to number (Figure 1B) but showed a small reduction in length (Figure 1C). We further generated Tulp3 ko 3T3-L1 preadipocyte and NIH 3T3 cells using CRISPR/Cas9 technology (Figure S1B). We observed that Arl13b ciliary localization was inhibited in Tulp3 ko 3T3-L1 and NIH 3T3 cells (Figure 1D-1E, Figure S1C-D), confirming that Tulp3 is required for Arl13b ciliary localization. While ciliation with respect to number was unaffected (Figure 1E), a small reduction in ciliary length was observed in Tulp3 ko 3T3-L1 cells (Figure 1F). Smoothened, a Tulp3 independent cargo (Mukhopadhyay et al., 2010), was trafficked to Tulp3 ko 3T3-L1 cells upon treatment with a Smoothened agonist SAG (Figure S1EF), ruling out generalized effects on localization from defects in transition zone (Chih et al., 2011).

Tulp3 belongs to tubby protein family consisting of four other members, Tubby, Tulp1, Tulp2 and Tulp4 (Mukhopadhyay and Jackson, 2011). Tulp3 is ubiquitously expressed, while Tubby is expressed only in brain and retina (Mukhopadhyay and Jackson, 2011). Tubby also binds to IFT-A, but to a lesser extent (Badgandi et al., 2017; Mukhopadhyay et al., 2010) and regulates ciliary targeting of GPCRs (Badgandi et al., 2017; Loktev and Jackson, 2013; Sun et al., 2012). Unlike kidney tubules (Hwang et al., 2019; Legue and Liem, 2019), we observed that 
ciliary localization of Arl13b was unaffected in Nestin-Cre; Tulp ${ }^{f / f}$ brain regions at P7 (caudate putamen/striatum, dentate gyrus and CA1 region of the hippocampus) (Figure 1G-H, Figure S2A-B) and in postnatal cerebellum lobes at P7 (Figure 1H). RNA transcripts of Tulp3 were reduced in Tulp3 cko brain (Figure 1I). Arl13b has also been reported to be unaffected in cilia of Tulp3 ko mouse neural tube (Ferent et al., 2019). Therefore, Arl13b is unaffected in Tulp3 deletions in brain and neural tube, likely from redundancy with Tubby.

\section{TULP3 determines entry of ARL13B into cilia}

Steady state levels of a protein in cilia can be regulated at multiple steps, including trafficking to cilia, removal from the compartment, or additional steps in cargo recycling (Mukhopadhyay et al., 2017). We previously demonstrated that Tulp3 determined entry of Gpr161 into cilia, rather than inhibiting removal from the compartment (Badgandi et al., 2017). Therefore, we tested the direct role of Tulp3 in Arl13b entry into cilia. We tested the dynamics of ciliary entry of stably expressed ARL13B that was tagged at the N-terminus with GFP-SNAP ( ${ }^{\text {GFP-SNAP }}{ }^{-R L} 13 B$ ) in control and Tulp3 ko inner medullary collecting duct (IMCD3) cells (Figure 1J), generated using CRISPR/Cas9 technology (Figure S1B). After blocking intracellular pools of GFP-SNAP tagged ARL13B using non fluorescent SNAP substrate, we tracked accumulation of newly trafficked SNAP-tagged proteins in cilia by immunofluorescence using fluorescent SNAP substrate (TMR-Star) at different time points. We detected an increase of up to $\sim 50 \%$ of mean steady state SNAP-labeled pools by $5 \mathrm{~h}$ in cilia of control cells. Such increase was strongly reduced in Tulp3 ko cells, suggesting inhibition of ciliary entry of ARL13B (Figure 1J, Figure S2C).

\section{TULP3 is required for enrichment of both farnesylated and myristoylated proteins in cilia} Arl13b acts as a GEF for Arl3 converting inactive Arl3-GDP to active Arl3-GTP (Gotthardt et al., 2015; Ivanova et al., 2017). Arl3 once activated promotes ciliary release of multiple lipidated cargoes including farnesylated proteins such as Inpp5e and myristoylated proteins such as Nphp3 and Cystin1 (Cys1) from their respective carrier proteins Pde6 $\delta$ (Humbert et al., 2012) and 
Unc119b (Wright et al., 2011), respectively (Figure 2A). As we observed that Arl13b ciliary trafficking was dependent on Tulp3, we asked if Tulp3 is also required for ciliary trafficking of Arl13b/Arl3 dependent cargoes. To answer this question, we stably expressed GFP-Stag (LAP) tagged INPP5E, NPHP3 (1-203 aa) or CYS1 (Figure 2B, 2D) in control or Tulp3 ko IMCD3 cells (Figure S1B). Compared to control, Tulp3 ko IMCD3 cells showed loss of endogenous Arl13b and GFPINPP5E ciliary localization (Figure 2B-C). Importantly, ciliary trafficking of Arl13b and GFPINPP5E were restored upon stably expressing ${ }^{\text {Myc }}$ TULP3 (Figure 2B-C) ruling out effects from nonspecific deletions in the genome. Total cellular levels of endogenous Arl13b protein were unaffected by immunoblotting in Tulp3 ko (Figure 2C). Localization of NPHP3 ${ }^{\text {GFP }}$ and CYS1 ${ }^{\text {GFP }}$ were reduced in intensities but were partially retained in cilia when quantified as cilia positive for respective proteins irrespective of decreased intensities (Figure 2D-F). Lkb1 is a farnesylated protein that is targeted to cilia by the pseudokinase STRAD $\beta$ (Mick et al., 2015). Together, they form a heterotrimer complex with the scaffolding protein MO25 (Zeqiraj et al., 2009). Lkb1 trafficking is therefore likely to be independent of Arl3. In agreement with this, ciliary localization of endogenous Lkb1 was only modestly reduced in Tulp3 ko with no significant decrease in ciliary intensities (Figure 2G-I), suggesting Lkb1 trafficking to be independent of Tulp3. We also observed a small decrease in ciliary length upon Tulp3 ko in IMCD3 cells that was partially rescued by stably expressing ${ }^{\text {Myc}}$ TULP3 (Figure $\mathbf{2 J}$ ). Thus, Tulp3 determines ciliary localization of Arl13b and Arl13b dependent lipidated cargoes.

To directly test effects from ARL13B on INPP5E localization in cilia, we stably expressed $\mathrm{N}$ term- or C term-GFP-Stag (LAP) tagged ARL13B in immortalized Arl13b $b^{h n n}$ MEFs (Figure S3) (Larkins et al., 2011). Arl13b $b^{\text {hnn }}$ embryos completely lack Arl13b (Caspary et al., 2007) and do

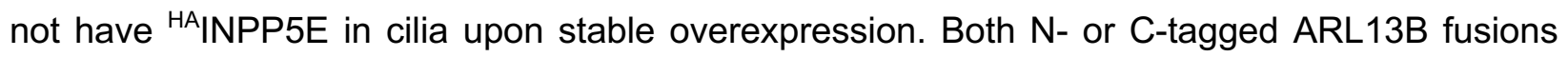

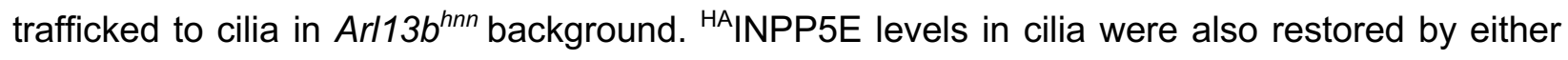
fusion, suggesting rescue from their stable expression (Figure S3). 


\section{Tulp3 is required for ciliary localization of lipidated proteins in murine kidneys}

Embryonic cko of Arl13b (Li et al., 2016; Seixas et al., 2016) and potential Arl13b regulated cargoes, Inpp5e (Hakim et al., 2016), Nphp3 (Bergmann et al., 2008) and Cys1 (Hou et al., 2002) cause mild cystic disease, phenocopying Tulp3 loss at similar timelines (Hwang et al., 2019). Therefore, we tested the role of Tulp3 in ciliary trafficking of lipidated cargoes in vivo in the mouse kidney. Tulp3 ko mice are embryonic lethal and die by age E13.5 (Norman et al., 2009). Therefore, we generated conditional Tulp3 knockouts using collecting duct-specific HoxB7-Cre (HoxB7-Cre;

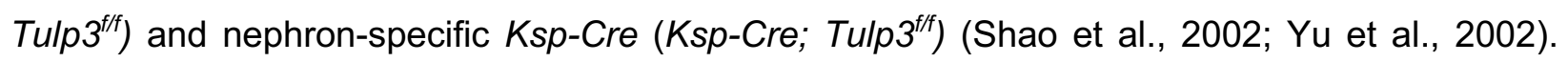
These mice develop progressive cystogenesis but survive till postnatal day 30 (P30) (Hwang et al., 2019). We previously showed that Arl13b and Inpp5e localized to collecting duct cilia and localization was reduced in both Hoxb7-Cre; Tulp ${ }^{f / f}$ and Ksp-Cre; Tulp $3^{f / f}$ collecting duct cilia by P5 (Hwang et al., 2019). Nphp3 localizes to the proximal inversin zone in cilia (Bennett et al., 2020) but localization in kidney collecting ducts is unknown. Lkb1 has been reported to localize to kidney collecting duct cilia (Viau et al., 2018). We found that both Nphp3 and Lkb1 were localized to collecting duct cilia in control mice (Figure 3A-B). However, both Hoxb7-Cre; Tulp3 f/f and Ksp-Cre; Tulp3 $3^{f / f}$ kidney collecting ducts had reduced ciliary localization of Nphp3 by P24, whereas Lkb1 was unaffected (and Figure 3A-B).

Importantly, we observed a significant difference in the kinetics of loss of these proteins from Tulp3 cko kidney cilia (Figure 3C). Arl13b is almost completely lost by P0, Inpp5e by P5 and Nphp3 by P24. This is consistent with our cell line data where Arl13b and INPP5E were the most significantly affected while NPHP3 and CYS1 were comparatively less affected (Figure 2). The extent of ciliogenesis was unaffected in Tulp3 cko collecting ducts as reported before (Figure 3D) (Hwang et al., 2019). Overall, our data using cell lines and murine kidneys strongly suggest that Tulp3 acts as a master regulator for trafficking of different classes of Arl13b regulated lipidated proteins to cilia. 
TULP3 traffics lipidated cargoes to cilia in a predominantly $\mathrm{PI}(4,5) \mathrm{P}_{2}$-independent but IFTA-dependent manner

We previously demonstrated that both $\mathrm{PI}(4,5) \mathrm{P}_{2}$ binding and IFT-A binding properties of TULP3 are required for its role in ciliary trafficking of GPCRs (Badgandi et al., 2017; Mukhopadhyay et al., 2010). We asked if $\mathrm{PI}(4,5) \mathrm{P}_{2}$ and IFT-A binding properties of TULP3 are also required for trafficking Arl13b and ${ }^{\mathrm{HA}}$ INPP5E to cilia. Two residues K268 and R270 in the C-terminal Tubby domain of TULP3 are required for interaction with $\mathrm{PI}(4,5) \mathrm{P}_{2}$ (Mukhopadhyay et al., 2010; Santagata et al., 2001), whereas conserved residues in an N-terminal $\alpha$-helical region of TULP3 are important for its interaction with IFT-A complex (Mukhopadhyay et al., 2010). We stably expressed mutant forms of ${ }^{\text {LAPTULP3 }}$ that were deficient in either $\mathrm{PI}(4,5) \mathrm{P}_{2}$ binding (K268A; R270A designated as TULP3 ${ }^{\text {KR }}$ ) (Mukhopadhyay et al., 2010; Santagata et al., 2001) or IFT-A binding (TULP3 ${ }^{\text {mut12 }}$ ) (Mukhopadhyay et al., 2010) or both $\mathrm{PI}(4,5) \mathrm{P}_{2}$ and IFT-A binding $\left(\right.$ Tulp3 $3^{\mathrm{KR} / \mathrm{mu} 12}$ ) in Tulp3 ko IMCD3 cells (Figure 4A). We observed that expression of TULP3 $3^{\text {mut12 }}$ or TULP $3^{K R / m u t 12}$ did not rescue ciliary trafficking of either Arl13b or ${ }^{\mathrm{HA}}$ INPP5E indicating that Tup3IFT-A binding is required for ciliary trafficking of lipidated cargoes (Figure 4B-D). Interestingly, expression of $T U L P 3^{K R}$ efficiently rescued ciliary trafficking of both Arl13b and ${ }^{\mathrm{HA}}$ INPP5E (Figure 4B-D), indicating that TULP3-PI(4,5) $\mathrm{P}_{2}$ binding is dispensable for lipidated cargo trafficking by TULP3. However, the ciliary intensities of both Arl13b and ${ }^{\mathrm{HA}}$ INPP5E were reduced in cells rescued by $T U L P 3^{K R}$, indicating a partial rescue (Figure 4D). In contrast, expression of TULP3 $3^{K R}$ did not rescue ciliary trafficking of Gpr161, a GPCR cargo of TULP3, similar to expression of TULP3 $3^{\text {mut12 }}$ or $T U L P 3^{K R / m u t 12}$ (Figure 4B-D), confirming that both $\mathrm{PI}(4,5) \mathrm{P}_{2}$ and IFT-A binding by TULP3 are required for ciliary trafficking of Gpr161 (Mukhopadhyay et al., 2010). Expression of $T U L P 3^{K R}$ also partially restored ciliary length in Tulp3 ko cells compared to expression of either TULP3 $^{\text {mut12 }}$ or TULP3 $3^{\text {KR/mut12 }}$ (Figure 4E). Recently, Han et. al. showed that $\mathrm{PI}(4,5) \mathrm{P}_{2}$ binding property of TULP3 is not required for ciliary trafficking of ARL13B and GPR161 (Han et al., 2019). Our results using stably expressed TULP3 WT and mutants, rather than transient expression by 
transfecting variants in the previous study (Han et al., 2019), suggest that TULP3 traffics Gpr161 in a $\mathrm{PI}(4,5) \mathrm{P}_{2}$ dependent manner and lipidated cargoes in a predominantly $\mathrm{PI}(4,5) \mathrm{P}_{2}$ independent manner.

\section{Proximity biotinylation assays determine ARL13B-TULP3 interactions}

As Tulp3 is required for ciliary trafficking of Arl13b, we tested if there is a physical association between the two proteins. We showed earlier that the ciliary localization sequence (CLS) fusions of transmembrane cargoes with CD8 $\alpha$ are in proximity to TULP3 using proximity biotinylation or cross linking in vivo. (Badgandi et al., 2017) Similarly, we co-expressed ARL13B or CLSs' fused with a promiscuous biotin ligase BirA* (BirA mutant R118G (Roux et al., 2012)) and ${ }^{\text {LAPTULP3 in }}$ TRex-293 cells (LAP, EGFP-TEV-Stag-X; Figure 5A). To remove background resulting from nonspecific biotinylation of GFP, we performed tandem affinity purifications of LAPTULP3. We observed that TULP3 was biotinylated by BirA*-ARL13B in comparison to a CD8 $\alpha$ control without any CLS (CD8 Linker; Figure 5B). The biotinylation of TULP3 in presence of ARL13B was at similar levels to that in presence of the fibrocystin CLS fused with CD8 $\alpha$, one of the transmembrane cargoes of TULP3 that we identified earlier (Badgandi et al., 2017). TULP3 has a conserved C-terminal tubby domain and an N-terminal region that contains IFT-A binding motif. The C-terminal tubby domain of TULP3, but not the $\mathrm{N}$-terminus, has been shown to be in proximity to its transmembrane cargoes (Badgandi et al., 2017). Similarly, we observed that the C-terminal tubby domain of TULP3, but not the $\mathrm{N}$ terminal region, was biotinylated by ARL13B (Figure 5B), indicating that ARL13B, like GPCR cargoes, interacts with the tubby domain.

\section{The Tubby domain of TULP3 binds directly to ARL13B}

TULP3 traffics ARL13B in a predominantly $\mathrm{PI}(4,5) \mathrm{P}_{2}$ independent manner unlike its GPCR cargoes. Therefore, we speculated that TULP3 binds to ARL13B efficiently compared to its GPCR cargoes. Further, TULP3 cargoes that are known to date are integral membrane proteins and we previously could not confirm direct interaction between these cargoes and TULP3. As ARL13B is 
a membrane associated protein, we tested if ARL13B interacts directly with TULP3 in vitro. We prepared pure ARL13B protein by in vitro translation (IVT) using TNT-SP6 wheat germ extract. In vitro translated ${ }^{M y c} A R L 13 B$ interacted with bacterially purified ${ }^{\text {GST }}$ TULP3 (Figure 5C). Excess ${ }^{\text {MBP }}$ TULP3 or ${ }^{\text {MBP }}$ Tubby, but not MBP alone, could compete out ${ }^{\text {Myc }}$ ARL13B binding to ${ }^{\text {GST }}$ TULP3 (Figure 5C), suggesting that conserved regions in Tubby family proteins might be involved in interaction with ARL13B. Furthermore, using bacterially purified GST tagged TULP3 N and C terminal truncations, we confirmed that the C-terminal tubby domain of TULP3 interacts with in vitro translated ${ }^{M y c} A R L 13 B$ (Figure 5D). Thus, the C-terminus tubby domain of TULP3 directly binds to ARL13B.

Our attempts to purify ARL13B from bacterial cells were not successful as we always saw multiple degradation products in our protein preparations. Thus, we purified full length and N-term fragment (1-298 aa; $\left.A R L 13 B^{N T}\right)$ of ARL13B from Sf9 insect cells using the FlexiBac system (Lemaitre et al., 2019). The Sf9 cell system also allows purification of proteins with posttranslational modifications such as palmitoylation (Mouillac et al., 1992; $\mathrm{Ng}$ et al., 1994). The

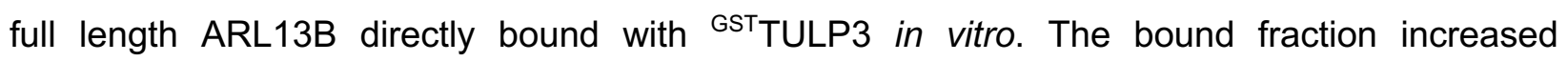
proportionally to the amount of ARL13B in the reactions (Figure 5E). Furthermore, such binding was also seen between the tubby domain of TULP3 $\left({ }^{\mathrm{GST}} \mathrm{TULP}^{\mathrm{CT}}\right.$ ) and ARL13B ${ }^{\mathrm{NT}}$ (Figure 5F), with bound fractions increasing proportionally to the levels of ARL13B ${ }^{N T}$ in the reactions. Thus, TULP3 directly binds through its tubby domain to ARL13B.

\section{A conserved lysine in the tubby domain of TULP3 is crucial for cargo interactions}

To identify potential cargo binding residues on TULP3 we performed proximity based biotinylation using BirA* tagged GPCR CLSs followed by mass spectrometry. We identified multiple lysine residues in the tubby domain of TULP3 that were in proximity to the GPCRs Gpr161 and MCHR1 in comparison to corresponding mutated CLSs that are non-ciliary (Figure S4A) (Badgandi et al., 2017). One of these lysine residues, K389 in human TULP3 corresponds to K407 in mouse Tulp3 
(Figure S4B) and was also identified as a Tulp $3^{K 407 l}$ allele in mice exhibiting perinatal lethality and kidney cystogenesis during later embryogenesis (Legue and Liem, 2019). This allele is hypomorphic to Tulp3 null that is embryonic lethal at E14.5 (Norman et al., 2009), but it phenocopies Tulp3 cko in the embryonic kidney that causes postnatal cystogenesis (Hwang et al., 2019). To test if this residue in TULP3 is required for cargo binding, we performed cell-based proximity biotinylation. Interestingly, we noticed that LAPTULP3 ${ }^{\mathrm{K} 3891}$ had significantly reduced proximity to ARL13B compared to ${ }^{\text {LAPTULP3 }}{ }^{W T}$ (Figure 6A). Furthermore, we observed that ${ }^{\text {GSTTTUP3 }}{ }^{\mathrm{K} 3891}$ had reduced direct binding with insect cell purified $\mathrm{ARL}^{13^{\mathrm{NT}}}$ (1-298 aa) in vitro

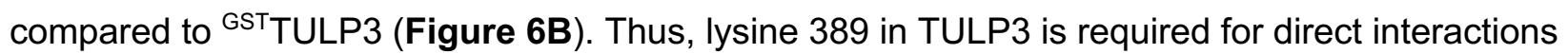
with ARL13B.

TULP3 has multiple other cellular roles including binding to the IFT-A core through the Nterminus, binding to phosphoinositides including $\mathrm{PI}(4,5) \mathrm{P}_{2}$ through the tubby domain, and ciliary localization (Mukhopadhyay et al., 2010). We identified most IFT-A complex proteins among interacting partners of stably expressed LAP-tagged TULP3 ${ }^{\mathrm{K} 3891}$ in RPE-hTERT cells using tandem affinity purification and mass spectrometry (Figure 6C-6D) and by immunoblotting for IFT139 (Figure 6E). We found that recombinant ${ }^{\text {GSTTTULP3 }}{ }^{\mathrm{K} 3891}$ also bound to select phosphoinositides including $\mathrm{PI}(4,5) \mathrm{P}_{2}$ on $\mathrm{PIP}$ blots similar to ${ }^{\mathrm{GST}} \mathrm{TULP}{ }^{\mathrm{WT}}$ as reported before (Figure 6F) (Mukhopadhyay et al., 2010). Finally, ${ }^{\text {LAPTULP3 }}{ }^{\mathrm{K} 389 \mathrm{I}}$ was localized to primary cilia upon stable expression in IMCD3 cells by immunofluorescence (Figure 6G) as for the wildtype protein (Mukhopadhyay et al., 2010). Thus, TULP3 ${ }^{\mathrm{K} 389 \mathrm{l}}$ had diminished cargo binding, whereas phosphoinositide binding, IFT-A binding and ciliary localization remained unaffected.

\section{Lysine 389 in TULP3 is required for ciliary trafficking of its cargoes}

We next tested if the K389 residue in TULP3 is important for ciliary cargo trafficking. We stably expressed TULP3 ${ }^{W T}$ or TULP3 $3^{K 3891}$ in Tulp3 ko IMCD3 cells and observed that WT, but not K389I,

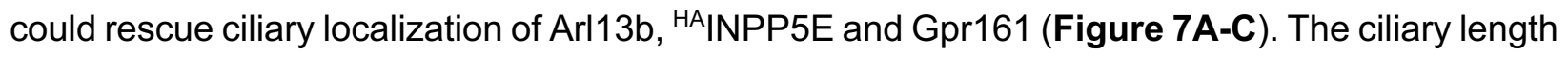


in $T U L P 3^{K 3891}$ expressing cells showed a small reduction in length compared to TULP3 ${ }^{W T}$ expressing cells (Figure 7D). Furthermore, endogenous ciliary pools of both Arl13b and Gpr161 were abrogated in MEFs from Tulp $3^{K 4071}$ mice (Figure 7E-F). Thus, the lysine 389 in TULP3 was critical for trafficking both GPCR and lipidated cargoes to cilia suggesting that a common interface in the tubby domain of TULP3 mediated trafficking of both transmembrane and membrane associated cargoes.

\section{The N-terminus of ARL13B mediates TULP3 binding}

Our earlier studies showed that for almost all TULP3 dependent cargoes, the ciliary targeting motifs are short sequence in the respective proteins. These sequences are in proximity to TULP3 and are both necessary and sufficient for ciliary trafficking by TULP3 (Badgandi et al., 2017). To dissect ciliary targeting of ARL13B by TULP3, we aimed at identifying ARL13B regions involved in TULP3 binding. ARL13B has a palmitoylation motif and GTPase domain followed by a coiledcoiled (CC) domain in its N-terminus along with RVxP motif and Proline Rich (PR) region in its Cterminus (Figure 8A). We tested various in vitro translated truncations of ${ }^{M y c} A R L 13 B$ for their capacity to bind with ${ }^{\text {GSTTULP3 }}$ and observed that the N-terminus of ARL13B corresponding to the GTPase and coiled coil domains (D2, 1-244 aa) binds to TULP3 stronger than the C-terminus alone (D5, 245-428 aa) (Figure 8A-B). As mentioned before, the insect cell purified N-terminus (residues 1-298) of GFPARL13B also binds to the bacterially purified C-terminal tubby domain of

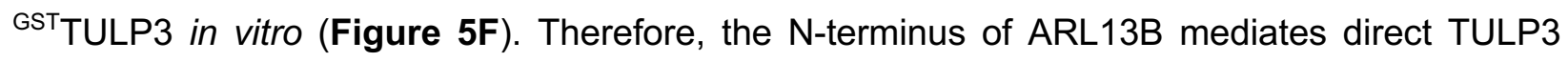
binding.

The C-term RVxP motif of ARL13B requires membrane anchoring for trafficking to cilia in a TULP3 independent manner

Previous studies reported the RVxP motif (sequence $\mathrm{R}^{357} \mathrm{VEP}$ ) in the C-terminus of mouse Arl13b (corresponding to $358-361 \mathrm{R}^{358} \mathrm{VEP}$ of human $\mathrm{ARL} 13 \mathrm{~B}$ ) as a potential ciliary localization signal (Mariani et al., 2016). As reported before (Gigante et al., 2020), we did not see the RVxP mutant 
protein in cilia in MEFs with knock-in at the endogenous RVxP locus (Arl13b $b^{\text {V358AN358A }}$ ) (Figure S5A). By cell-based proximity biotinylation assay, we noted that $A R L 13 B^{\vee 359 A}$ interacted with TULP3 efficiently when compared with the wild type ARL13B (Figure S5B). Similarly, in vitro binding between ${ }^{\text {GST }}$ TULP3 and in vitro translated ${ }^{M y c} A R L 13 B^{V 359 A}$ was similar compared to wild type Myc ARL13B (Figure S5C). Thus, ARL13B RVxP mutant failed to traffic to cilia despite efficient TULP3 binding.

We next tested additional requirements for the RVxP motif to traffic to cilia. A $\beta 1$ IntegrinHaloTag-ARL13B-C-GFP (IA-GFP) traffics to cilia (Su et al., 2013). In this construct the C-term region of human ARL13B containing the RVxP motif (355-428 aa) is anchored to the cell surface by fusion to the transmembrane domain of human $\beta 1$ integrin (Svendsen et al., 2008). By transfecting IA-GFP in Tulp3 ko NIH 3T3 cells, we found that this construct is trafficked to cilia independent of Tulp3 (Figure S5D), as expected from the corresponding C-terminus domain of ARL13B (D5 and D6, 362-428) not binding to TULP3. (Figure 8A-B). The $\beta 1$ Integrin-HaloTagGFP was not by itself able to traffic to cilia (Figure S5D). As reported earlier (Larkins et al., 2011), the ARL13B D5 fragment fused with C-terminal LAP was not trafficked to cilia when expressed in Arl13 $b^{h n n}$ MEFs (Figure 8A, S5E). Therefore, membrane anchoring is required for the RVxP motif containing C-term ARL13B fragment to traffic to cilia and is independent of TULP3.

\section{An N-terminal amphipathic helix in ARL13B binds to TULP3}

Based on other ARF family proteins (Donaldson and Jackson, 2011; Randazzo et al., 1995), ARL13B has a lipid-sensitive clamp provided by an amphipathic helix at the $\mathrm{N}$ terminus preceding the GTPase domain. Thus, the first 19 residues of human/mouse ARL13B (Hori et al., 2008; Ivanova et al., 2017) and corresponding residues in the Chlamydomonas protein (Gotthardt et al., 2015) were not included when performing GTP binding assays and structural studies. We already had the first 19 aa in our insect cell purified ${ }^{\text {GFP }}$ ARL13B protein that showed binding with TULP3 (Figure 5E-F and 6B). We next tested if the C-terminal region fused with the short $\mathrm{N}$-terminal 
amphipathic helix (D16, Figure 8A) interacts with TULP3. We observed that this construct bound to TULP3 as efficiently as the full-length or the N-terminus (D2) in vitro (Figure 8B).

The amphipathic conserved helix flanks the palmitoylation site (Figure $\mathbf{8 C}$ ). We wondered if the amphipathic helix was responsible for TULP3 binding. We first tested the helix deleted ${ }^{M y c} A R L 13 B^{\Delta 19}$ in our in vitro translated protein binding assays (Figure $8 \mathrm{C}$ ). Remarkably, this

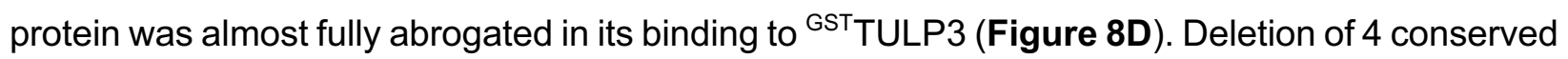
aa preceding the palmitoylation site $(\Delta 1$; Figure $8 \mathrm{C})$ and but not of seven conserved aa following the palmitoylation site ( $\Delta 2$; Figure $8 \mathrm{C}$ ) in ${ }^{\mathrm{Myc}} \mathrm{ARL} 13 \mathrm{~B}$ modestly reduced binding to ${ }^{\mathrm{GST} T U L P 3}$ (Figure 8D). Finally, a double deletion mutant of these regions in the full-length protein $(\Delta 1,2$;

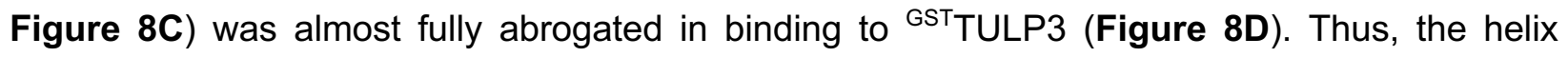
flanking the palmitoylation site mediates TULP3 binding.

\section{The amphipathic helix directs trafficking to cilia even in the absence of palmitoylation and RVxP motif}

To directly test role of TULP3 binding in ciliary localization of ARL13B, we stably expressed different ARL13B fragments and mutants fused with C-terminal LAP in the $A r 13 b^{h n n}$ background (Figure $8 \mathrm{E}$ ). We chose to test expression in the $A r 13 b^{h n n}$ background as self-association of ARL13B through its $\mathrm{N}$-terminal domain has been reported using Co-IP experiments (Hori et al., 2008). We also co-expressed HAINPP5E to check for restoration of ARL13B function in the Ar13b $b^{h n n}$ background (Figure 8E).

We first tested the D2 fragment for trafficking to cilia in the Arl13b $b^{h n}$ background. Consistent with our TULP3 binding data (Figure 8B), we noticed ciliary trafficking of the D2

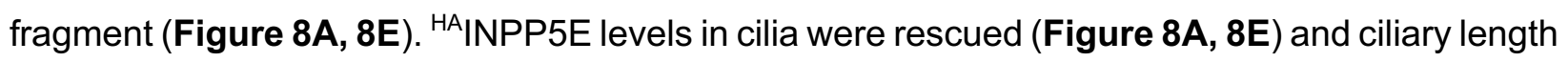
was partially rescued compared to the Ar13b $b^{h n}$ MEFs (Figure S5F). Thus, the N-terminus of ARL13B (up to the CC domain) containing the TULP3 binding amphipathic helix traffics to cilia and is functional. 
A full length palmitoylation deficient mutant upon transient expression by transfection has been reported to be only partially trafficked to cilia in the $A r l 13 b^{h n n}$ background (Mariani et al., 2016). Similar motif changes in C. elegans, however, does not prevent ARL13B trafficking to proximal segments of phasmid cilia, although promoting mislocalization to the cell body (Cevik et al., 2013). Upon stable expression in $A r l 13 b^{h n n}$, we found that the ARL $13 B^{\mathrm{C} 89 \mathrm{~S}}$ mutant fused with

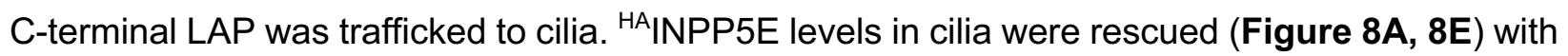
partial rescue of ciliary length compared to the $A r 13 b^{h n n}$ MEFs (Figure S5F). Thus, the full length ARL13B is localized to cilia despite lacking in palmitoylation. To rule out any role of palmitoylation in the $D 2$ context, we tested if the palmitoylation deficient $D 2$ fragment $\left(D 2^{\mathrm{C} / 9 \mathrm{~S}}\right)$ trafficked to cilia (Figure 8E). We found that D2 ${ }^{\mathrm{C} 8,9 \mathrm{~S}}$ still trafficked to cilia in the Arl13b $b^{\text {hnn }}$ background (Figure 8E). HAINPP5E levels in cilia were rescued Figure 8A, 8E), but ciliary length was unchanged compared to the $A r 13 b^{h n n}$ MEFs (Figure S5F). Thus, the amphipathic helix in ARL13B mediates binding to TULP3 and directs trafficking to cilia even in the absence of palmitoylation and the RVxP motif.

Finally, we generated a full length ARL13B fusion that lacked in TULP3 binding (ARL13B ${ }^{\Delta 1}$, ${ }^{\Delta 2}$ ) without disrupting the palmitoylation motif (Ren et al., 2008; Weng et al., 2017) (Figure 8C). This mutant was not trafficked to cilia (Figure 8E). Correspondingly, ${ }^{\mathrm{HA}}$ INPP5E levels in cilia were not rescued (Figure 8E). Thus, TULP3 binding is absolutely required for trafficking of the full length $A R L 13 B$ to cilia. 


\section{Discussion}

$\mathrm{ARL} 13 \mathrm{~B}$ is indisputably one the most used ciliary membrane markers since its discovery more than a decade ago (Caspary et al., 2007; Sun et al., 2004). The highly enriched ciliary pools of ARL13B seen in multiple tissues and ciliated cells contributes to the universality of its use as a ciliary marker. However, the mechanisms that traffic ARL13B to cilia are not well understood, and whether a defined region in ARL13B by itself suffices for ciliary trafficking is not known. We show that TULP3 is a master regulator for trafficking ARL13B to cilia without affecting total cellular pools. ARL13B trafficking to cilia is primarily regulated by direct binding to TULP3 and TULP3's binding to the IFT-A core complex. An N-terminus amphipathic helix that flanks the palmitoylation site and precedes the GTPase domain mediates binding of ARL13B to TULP3 and directs trafficking to cilia even in the absence of palmitoylation and the RVxP sorting motif. Unlike trafficking of GPCRs that requires both IFT-A and $\mathrm{PI}(4,5) \mathrm{P}_{2}$ binding by TULP3 (Mukhopadhyay et al., 2010), the binding between ARL13B and TULP3 is of sufficient strength to traffic ARL13B and ARL13B-dependent lipidated cargoes even without $\mathrm{PI}(4,5) \mathrm{P}_{2}$ anchoring by TULP3. A conserved lysine in TULP3's tubby domain (lysine 389 in human TULP3) mediates direct ARL13B binding and determines trafficking of both lipidated and transmembrane cargoes. Overall, our results suggest that TULP3 binding to short sequences in diverse cargoes is mediated by a shared region in the tubby domain.

\section{TULP3 enriches ARL13B-dependent lipidated proteins in cilia}

We demonstrate that Arl3-dependent farnesylated (Inpp5e) and myristoylated (Nphp3, Cys1) proteins are regulated by Tulp for ciliary localization. The farnesylated protein Lkb1, which is Arl3 independent for trafficking to cilia, is not Tulp3 regulated. Inpp5e loss in cilia of Tulp3 cko kidney collecting duct epithelia accompanies cystogenesis and follows Arl13b depletion. Nphp3 and Cys 1 are reduced in ciliary intensity but retain weak levels in cilia in Tulp3 ko cells. Nphp3 is also slow to be depleted from Tulp3 cko kidney collecting duct epithelial cilia. The effectiveness for 
complete depletion of Inpp5e in cilia in Tulp3 ko might be related to direct binding between Arl13b and Inpp5e and requirement of such binding for effective ciliary retention of Inpp5e (Humbert et al., 2012; Qiu et al., 2021). Nphp3 is concentrated in the proximal ciliary inversin compartment by binding to Nek8 and Anks6 that are required downstream of Inversin for Nphp3 localization (Bennett et al., 2020). Such binding might promote some retention of Nphp3 even in the absence of Tulp3. Nonetheless, the depletion of lipidated cargoes with distinct kinetics from kidney epithelial cilia following Tulp3 deletion strongly suggests their differential roles in renal cystogenesis from Arl13b (Li et al., 2016; Seixas et al., 2016) or Tulp3 loss during embryogenesis (Hwang et al., 2019; Legue and Liem, 2019). The ciliary levels of Arl13b are however unaffected upon Tulp3 deletion in brain/cerebellum and neural tube (Ferent et al., 2019), likely from redundancy between Tulp3 and Tubby in trafficking Arl13b. Such redundancy is also seen in trafficking of a subset of GPCRs by tubby in brain (Badgandi et al., 2017).

\section{An amphipathic helix determines ciliary localization of ARL13B}

The amphipathic helix region is a signature feature in the ARF family proteins (Donaldson and Jackson, 2011; Randazzo et al., 1995) that ensures that they are associated with the membrane in GTP bound form (Antonny et al., 1997). This region had been previously omitted when performing GTP binding assays and structural studies using human, mouse (Hori et al., 2008; Ivanova et al., 2017) or Chlamydomonas ARL13B. We infer that the amphipathic helix is required for trafficking to cilia irrespective of palmitoylation and even in presence of intact RVxP motif from the following observations. First, a full length $A R L 13 B$ mutant $\left(A R L 13 B^{\Delta 1, \Delta 2}\right)$ that does not bind to TULP3 but retains palmitoylation and RVxP motifs is not trafficked to cilia, suggesting TULP3 binding to be absolutely required for trafficking of the full length ARL13B to cilia. Second, an Nterminal fragment of ARL13B including just the helix, GTPase and coiled coil domains (D2, 1-244 aa) and lacking the RVxP motif traffics to cilia and rescues INPP5E levels in cilia of Arl13b null 
cells. This N-terminal fragment, even upon being non-palmitoylated, traffics into cilia, suggesting that only TULP3 binding is required for ciliary trafficking of this RVxP motif lacking fragment.

\section{The RVxP motif cooperates with ARL13B bound TULP3-IFT-A to increase ciliary delivery}

The C-terminal RVxP motif in ARL13B was initially discovered to be involved in post-Golgi trafficking of Rhodopsin (Deretic et al., 2005). We find that the ARL13B mutated in the RVxP motif $\left(A R L 13 B^{V 359 A}\right)$ retained binding to TULP3 and proximity with TULP3 in vivo. However, despite TULP3 binding, the mutant protein was not trafficked to cilia in the Arl13b $b^{V 358 A N 358 A}$ MEFs. This result places the RVxP mechanism downstream of TULP3-IFT-A core coupling. We show that the RVxP motif by itself can traffic to cilia independent of TULP3, but only if provided with a membrane anchor (as provided by the $\beta 1$ integrin in the IA-GFP chimera). While the actual mechanism of the RVxP motif in ARL13B is not known, for secretory proteins such as Rhodopsin, interaction between this motif and ARF4 has been proposed to be important for post-Golgi trafficking (Wang et al., 2012). Despite being cytosolic, Arl13b localizes to tubular-vesicular structures of the Arf6and Rab22a-dependent recycling pathway (Barral et al., 2012). While ARF4 is seen among ARL13B interacting proteins in human kidney epithelial cells in an unbiased proximity-dependent biotinylation screen (He et al., 2018), the relevance of ARF4 and secretory pathway components in ARL13B trafficking to cilia would require further study. Both the N-terminus ARL13B D2 fragment without RVxP motif, and the non-palmitoylated full length ARL13B partially rescued ciliary length in Arl13b null cells. Levels of Arl13b correspond with ciliary length by inducing membrane protrusion and axonemal extension (Lu et al., 2015). Therefore, in addition to the TULP3 binding amphipathic helix, other motifs, such as RVxP and palmitoylation site, ensure the full complement of ARL13B in cilia and determine steady state ciliary length. Differential levels of Arl13b and downstream lipidated proteins in cilia could underlie the context specific requirements of ciliary pools of Arl13b in the kidney tubules (Duldulao et al., 2009) vs the neural tube (Gigante et al., 2020). 


\section{Role of IFT-A in ARL13B trafficking to cilia}

Apart from our results showing that TULP3-IFT-A core binding is required for ARL13B trafficking to cilia, evidence from other labs also suggests an important role for IFT-A core subunits in ARL13B ciliary localization. The IFT-A core comprises of IFT144, IFT122 and IFT140, whereas IFT43, IFT139 and IFT121 are peripheral subunits (Behal et al., 2012; Hirano et al., 2017; Mukhopadhyay et al., 2010). ARL13B levels in cilia are reduced in both IFT122 (Takahara et al., 2018) and IFT144 (Kobayashi et al., 2021) knockout cell lines. A recent proteomics study showed that the flagellar levels of multiple ARF family GTPases such as ARL13B, and other myristoylated and farnesylated proteins were reduced in an IFT-140 null Chlamydomonas mutant (Picariello et al., 2019). Whether tubby family proteins (Stolc et al., 2005; VanderWaal Mills, 2011) underlie the anterograde trafficking of lipidated proteins by IFT-140 in Chlamydomonas is currently unclear. Other than the IFT-A core-TULP3 interactions, the IFT121 peripheral subunit is also required for trafficking of GPCRs, ARL13B and SMO (Fu et al., 2016). Such dependance is abrogated in the absence of F-actin depolymerization by cytochalasin D (Fu et al., 2016), suggesting that IFT121 delivers cargoes to cilia by coordinating with actin binding proteins in the face of an intact filamentous actin network. As SMO trafficking to cilia is TULP3-IFT-A core independent (Mukhopadhyay et al., 2010; Norman et al., 2009; Qin et al., 2011), the role of IFT121 peripheral subunit in trafficking SMO, and by extension ARL13B and GPCRs, is unrelated to TULP3-IFT-A core interactions.

\section{A shared tubby domain interface in cargo trafficking by TULP3}

We find a site distinct from the $\mathrm{PI}(4,5) \mathrm{P}_{2}$ binding site in the tubby domain mediating cargo binding by TULP3. Direct binding between the TULP3 ${ }^{\mathrm{K} 3891}$ tubby domain mutant and ARL13B is reduced, as is proximity inside cells using biotinylation assays compared to wild type TULP3. $\mathrm{PI}(4,5) \mathrm{P}_{2}$ binding by the tubby domain is unaffected in TULP3 ${ }^{\mathrm{K} 3891}$, as is ciliary trafficking that is mediated by IFT-A core binding to TULP3 N-terminus (Mukhopadhyay et al., 2010). Strikingly, an equivalent 
mouse mutation in Tulp3 (K407I) prevents trafficking of Arl13b and Gpr161 to cilia in cultured cells. Also, Arl13b trafficking to kidney epithelial cilia is affected in Tulp3 ${ }^{K 407 I}$ E18.5 kidneys (Legue and Liem, 2019). Our experiments showing direct binding between purified TULP3 and ARL13B and the discovery of an amphipathic helix in N-term of ARL13B mediating TULP3 binding, suggest a short sequence in coupling with TULP3 tubby domain. Similar diverse CLSs from cilia-targeted GPCRs and fibrocystin are also required in the membrane-bound context for proximity between cargoes and TULP3. As ciliary trafficking of both Arl13b and Gpr161 are disrupted in the Tulp3 ${ }^{K 407 I}$ MEFs, TULP3 binding to short sequences in either protein is likely to be mediated by this region in the tubby domain. The minimal requirement of these diverse sequences might be to generate secondary structure, such as for the amphipathic helix in ARL13B. Drugging this interaction domain could provide therapeutics in ciliary trafficking-regulated diseases such as polycystic kidney disease and peripheral obesity. 


\section{Materials and methods}

\section{Mouse strains}

All mice were housed at the Animal Resource Center of the University of Texas Southwestern (UTSW) Medical Center. All protocols were approved by the UTSW Institutional Animal Care and Use Committee. Mice were housed in standard cages that contained three to five mice per cage, with water and standard diet ad libitum and a $12 \mathrm{~h}$ light/dark cycle. Both male and female mice were analyzed in all experiments. Nestin-Cre mice (Stock No. 003771) were obtained from Jackson Laboratory (Bar Harbor, ME) (Tronche et al., 1999). HoxB7-Cre and Ksp-Cre mice were obtained from O'Brien Kidney Research Core of UT Southwestern. ES cells targeting the second exon of Tulp3 from EUCOMM (HEPD 0508-5-B01) were used to generate the floxed mice that were obtained from MRC, Harwell as described before (Hwang et al., 2019). For genotyping of Tulp3 ${ }^{\text {f/f }}$ mice, we used the following primers: (a) Tulp3-5'Cas-WTF (5' CCA TTT GTG AGG GTT GCT TT 3') and Tulp3-Crit-WTR (5' GCT AAC ACA AGC CCA TGC TA 3') to detect wild type band (256bp) and floxed band (450 bp), and (b) Tulp3-F1 (5' AAG GCG CAT AAC GAT ACC AC 3') and Tulp3-R1 (5' ACT GAT GGC GAG CTC AGA CC 3') to detect the deletion. We did not notice any difference between wild type or heterozygous animals for Tulp3 floxed alleles with or without Cre recombinase, and thus were all included as littermate controls as mentioned in respective figure legends. For genotyping $\mathrm{Cre}$, following primers were used to detect transgene band: Cre-F (5' AAT GCT GTC ACT TGG TCG TGG C 3'), Cre-R (5' GAA AAT GCT TCT GTC CGT TTG C 3').

\section{Antibodies and reagents}

Anti-Arl13b rabbit polyclonal was from Tamara Caspary (Emory University School of Medicine) (Caspary et al., 2007), Anti-Smo rabbit polyclonal was from Kathryn Anderson (Memorial Sloan Kettering Cancer Center, New York, NY) (Ocbina and Anderson, 2008). Affinity-purified rabbit polyclonal antibody against TULP3 (Mukhopadhyay et al., 2010) and Gpr161 has been described previously (Pal et al., 2016). Anti-Ift139 (Thm1) antibody was from Dr. David Beier (Tran et al., 2008). Commercial antibodies used were against Arl13b (IF, N295B/66 NeuroMab; WB, Proteintech 17711-1-AP), GFP (IF, Abcam ab13970; WB, Abcam ab290), hFAB Rhodamine AntiTubulin (Bio-Rad; 12004166), acetylated a-tubulin (mAb 6-11B-1 T6793; Sigma-Aldrich;), S tag (EMD Millipore; MAC112), IRDye 680RD Streptavidin (LI-COR; 926-68079) Myc (Abcam; ab9132), HA (Rat polyclonal;), Flag (Abcam; ab1257;), MBP (New England Biolabs, Inc.), GST (Sigma; G1160) and AC3 (LifeSpan BioSciences; LS-C204505), Aqp2 (1:500 A7310 Sigma rabbit polyclonal; SC515770, Santa Cruz Biotechnology mouse IgG1), Inpp5e (Proteintech; 17797-1AP), Nphp3 (Proteintech; 22026-1-AP), Lkb1 (Cell Signaling Technologies; 13031T). Fluorescent secondary antibodies for immunofluorescence were from the Jackson Immuno Research Laboratories, Inc., and IRDye 680RD, IRDye 800CW secondary antibodies, and neutravidin 680RD for immunoblotting were from LI-COR Biosciences. Reagents used in this study included PIP Strips Membranes (Thermo Fisher; P23751) and Biotin (Sigma; B4639).

\section{Plasmids}

pG-LAP1 (pCDNA5/FRT/TO-EGFP-TEV-Stag-X) and pG-LAP5 (pEFa-X-Stag-PreScissionEGFP) were from Addgene (Torres et al., 2009). pENTR223-ARL13B fusion construct was from DNASU (HsCD00511796), pENTR221-INPP5E construct was from Life technologies (IOH40212), NPHP3 (encoding 1-203 aa) were synthesized commercially from Geneart (Life Technologies) and Gateway PLUS shuttle clone for CYS1 was from GeneCopoeia (GC-Y0203- 
CF). N or $\mathrm{C}$ terminal LAP tagged retroviral constructs of full length and truncations of TULP3, INPP5E, NPHP3, CYS1 and ARL13B were generated by Gateway cloning into a gatewaytized LAP1 or LAP5 version of pBABE, respectively. Lentiviral constructs of Myc-TULP3 and HAINPP5E were generated by gateway cloning into $\mathrm{PQXIN-Myc} \mathrm{and} \mathrm{PQXIN-HA}$ destination vectors, respectively. Single or multiple amino acid mutants of TULP3 and ARL13B were generated by Q5 site directed mutagenesis (New England Biolabs). For biotinylation experiments, DEST-pcDNA5BirA-FLAG N- or C-term destination vectors were from C. Gingras, The Lunenfeld-Tanenbaum Research Institute at Mount Sinai Hospital, Toronto, Canada. Myc tagged full-length and truncated ARL13B fragments were generated by in vitro translation using TNT Sp6 high-yield wheat germ protein expression system (Promega; L3261) from pCS2-Myc-ARL13B vectors generated by gateway cloning. FL and N-terminus (1-298 aa) of ARL13B was cloned into pOCC29 (MBP-TEV-GFP-insert-TEV-His) baculovirus vector for insect cell expression (Lemaitre et al., 2019). GST or MBP tagged proteins for bacterial purification were cloned into pGEX or pMalC2 vectors, respectively. GFP-SNAP-ARL13B was generated by cloning SNAP into a BstB1 site of the S-peptide in an N terminal LAP tagged ARL13B retroviral construct (pBABE). A $\beta 1$ IntegrinHaloTag-ARL13B-C-GFP (IA-GFP) was from Takanari Inoue, Johns Hopkins University (Su et al., 2013). The $\beta 1$ Integrin-HaloTag-GFP without ARL13B-C terminus (I-GFP) was generated by Q5 site directed mutagenesis (New England Biolabs).

\section{Cell culture and generation of stable cell lines}

RPE hTERT cells were grown in DMEM F12 media with $10 \%$ FBS (Sigma-Aldrich), $0.05 \mathrm{mg} / \mathrm{ml}$ penicillin, $0.05 \mathrm{mg} / \mathrm{ml}$ streptomycin, and $4.5 \mathrm{mM}$ glutamine. T-Rex-293 (Invitrogen), IMCD3 FlpIn, and Phoenix A (PhA) cells (Indiana University National Gene Vector Biorepository) were cultured in DMEM high glucose (Sigma-Aldrich; supplemented with $10 \%$ cosmic serum, 0.05 $\mathrm{mg} / \mathrm{ml}$ penicillin, $0.05 \mathrm{mg} / \mathrm{ml}$ streptomycin, and $4.5 \mathrm{mM}$ glutamine). Transfection of plasmids was done with Polyfect (QIAGEN) or Polyethylenimine (PEI) max. Stable cell lines were generated by retroviral infection or transfection. In many cases, stable lines were flow sorted and further selected for GFP. Control, Tulp3 ko and Tulp $3^{K 4071}$ MEFs were from E13.5 embryos (Legue and Liem, 2019). Immortalized wildtype, Arl13b $b^{V 358 A}$ and $A r l 13 b^{h n n}$ MEFs were gifts from Tamara Caspary (Gigante et al., 2020).

\section{Generation of Tulp3 knock out cell lines}

CRISPR/Cas9 knockout lines for Tulp3 were generated in IMCD3 FIp-In (Invitrogen), NIH 3T3 Flp-In and 3T3-L1 (gift of Peter Michaely, UT Southwestern) cells by using guide RNA targeting sequences caccgACGTCGCTGCGAGGCATCTG and caccgTGGCTTTAACCTTCGCAGCC targeting exon 3 in mouse Tulp3. Single clones were isolated using serial dilution method. Clonal lines were tested for knockout by Sanger sequencing and immunoblotting for Tulp3.

\section{Reverse transcription and quantitative PCR.}

RNA was extracted from paraffin embedded brain sections using deparaffinization silution (Qiagen Cat \#19093) and RNA extraction using GenElute mammalian total RNA purification kit (RTN350; Sigma). qRT-PCR was performed with Kicqstart One-Step Probe RT-qPCR ReadyMix (KCQS07; Sigma). Inventoried TaqMan probes for qRT-PCR from Applied Biosystems were used for human TULP3 and GAPDH. Inventoried probes for Gpr161, and Gapdh were. Reactions were run in CFX96 Real time System (Bio Rad).

\section{Immunofluorescence of cultured cells and microscopy}


Cells were cultured on coverslips until confluent and starved for indicated periods. Cells were fixed with 4\% PFA. After blocking with 5\% normal donkey serum, the cells were incubated with primary antibody solutions for $1 \mathrm{~h}$ at room temperature followed by treatment with secondary antibodies for 30 min along with Hoechst 33342 (Invitrogen). The coverslips were mounted using Fluoromount G (SouthernBiotech). Images were acquired on a microscope (Axiolmager.Z1; ZEISS), a sCMOS camera (PCO Edge; BioVision Technologies), and Plan Apochromat objectives $(10 \times / 0.45 \mathrm{NA} ; 20 \times / 0.8 \mathrm{NA} ; 40 \times / 1.3 \mathrm{NA}$ oil; and $63 \times / 1.4 \mathrm{NA}$ oil) controlled using Micro-Manager software (University of California, San Francisco) at room temperature. Between 8 and $20 \mathrm{z}$ sections at $0.5-0.8-\mu \mathrm{m}$ intervals were acquired. For quantitative analysis of ciliary localization, stacks of images were acquired from three to eight consecutive fields with confluent cells by looking into the DAPI channel, and percentages of protein-positive ciliated cells were counted. Maximal projections from images of stacks were exported from ImageJ/Fiji (National Institutes of Health) using a custom-written macro (from M. Mettlen, University of Texas Southwestern Medical Center, Dallas, TX) using similar parameters (image intensity and contrast) for image files from the same experiment. For measuring ciliary pixel intensities, image stacks were acquired with $z$ sections at $0.5-\mu \mathrm{m}$ intervals. An image interval with maximal intensity was chosen, and cilia were demarcated with a region of interest using fluorescence signal for acetylated a-tubulin. The mean pixel intensities for the corresponding protein were exported from ImageJ/Fiji. SNAP experiments were performed as described earlier (Follit and Pazour, 2013; Keppler et al., 2003). Briefly, cells on coverslips were serum starved for 36 hours to induce ciliation. $0.05 \mu \mathrm{M}$ BG-Block (NEB, S9106) was added for 30 min after which the coverslips were washed in serum starvation media and fixed at different time points. Cells were immunostained with indicated antibodies. $0.3 \mu \mathrm{M}$ fluorescent SNAP substrate (TMR Star, NEB, S9105) was added along with the primary antibodies

\section{Tissue processing, immunostaining, and microscopy}

Mice were perfused with PBS, and the brains and kidneys were dissected and fixed in $4 \%$ paraformaldehyde overnight at $4^{\circ} \mathrm{C}$ and processed for paraffin embedding and sectioning. For paraffin sectioning, tissues were processed over a 12-hour period using a Thermo-Fisher Excelsior Automated Tissue Processor (A82300001; ThermoFisher Scientific), which dehydrated the tissues through 6 ethanol concentrations, from $50 \%$ ethanol to $100 \%$ ethanol, cleared through 3 changes of xylene, and infiltrated with wax through 3 Paraplast Plus paraffin baths (39602004; Leica). Samples were embedded in Paraplast Plus using paraffin-filled stainless steel base molds and a Thermo-Shandon Histocenter 2 Embedding Workstation (6400012D; ThermoFisher Scientific). The tissues were then cut in $5 \mu \mathrm{m}$ thick sections, deparafinned and treated with microwave in Antigen Retrieval citra solution (HK086-9K; BioGenex. Fremont, CA) for $10 \mathrm{~min}$. Sections were then blocked using blocking buffer (1\% normal donkey serum [Jackson immunoResearch, West Grove, PA], in PBS) for 1 hour at room temperature. Sections were incubated with primary antibodies against the following antigens; overnight at room temperature or 4C: Acetylated tubulin (1:500, T6793; Sigma mouse IgG2b) Arl13b (1:500, N295B/66; NeuroMab Facility), Aqp2 (1:500 A7310 Sigma rabbit polyclonal; SC515770, Santa Cruz Biotechnology mouse lgG1), Inpp5e (1:500 17797-1-AP; Proteintech), Nphp3 (1:500 Proteintech; 22026-1-AP) or Lkb1 (1:500 Cell Signaling Technologies; 13031T). After three PBS washes, the sections were incubated in secondary antibodies (Alexa Fluor 488-, 555-, 594-, 647- conjugated secondary antibodies, or anti-mouse IgG isotype-specific secondary antibodies; 1:500; Life Technologies, Carlsbad, CA or Jackson ImmunoResearch). Cell nuclei were stained with DAPI (Sigma) or Hoechst 33342 (Life technologies). Slides were mounted with Fluoromount-G (010001; Southern Biotech) and images were acquired with a Zeiss Axiolmager.Z1 microscope or a Zeiss LSM780 confocal microscope. 


\section{Proximity biotinylation experiments}

T-Rex-293 cells were co-transfected with 5-7.5 $\mu \mathrm{g}$ each of LapN-TULP3 or LapN-TULP3 mutants/fragments and pDEST-pcDNA5-BirA-FLAG N/C-term expressing ARL13B or the CD8 linker-BirA or FibrocystinCLS-BirA fusion controls. The media was supplemented with $20 \mu \mathrm{M}$ biotin for 8-12 hours after transfection. Cells were harvested using PBS with 2 mM EDTA and 2 $\mathrm{mM}$ EGTA $48 \mathrm{~h}$ after transfection. Cells were lysed by resuspending and nutating for $20 \mathrm{~min}$ in 50 $\mathrm{mM}$ Tris- $\mathrm{HCl}, \mathrm{pH}$ 7.4, $200 \mathrm{mM} \mathrm{KCl}, 1 \mathrm{mM} \mathrm{MgCl}$, $1 \mathrm{mM}$ EGTA, 10\% glycerol, $1 \mathrm{mM}$ DTT, 0.6\% IGEPAL CA-630, $1 \mathrm{mM} \mathrm{AEBSF}$, and $0.01 \mathrm{mg} / \mathrm{ml}$ each of leupeptin, pepstatin, and chymostatin. Lysates were centrifuged at 12,000 gfor $10 \mathrm{~min}$ followed by tandem IPs (Fig. 5). In brief, the GFP immunoprecipitates were first digested with TEV protease for $16 \mathrm{~h}$ at $4^{\circ} \mathrm{C}$. The supernatants were subjected to secondary IP with S protein agarose. The resulting secondary IPs were analyzed by Western blotting. Blots were probed with antibodies against S tag (mouse monoclonal MAC112) and Flag (goat polyclonal ab1257) followed by visualization using IRDye-tagged secondary antibodies. IRdye-tagged streptavidin was used for confirming biotinylation signal on the blots.

\section{In vitro binding experiments:}

GST tagged proteins $(20 \mu \mathrm{g})$ were incubated with $30 \mu \mathrm{L}$ packed volume of glutathione sepharose beads in LAP150N (50 mM HEPES pH 7.4, 150 mM KCl, 1 mM EGTA, 1 mM MgCl, $10 \%$ glycerol, $0.05 \%$ NP-40) buffer at $4^{\circ} \mathrm{C}$ for $2-4$ hours. Beads were washed with LAP150N three times and then incubated with increasing amounts of IVT Myc-ARL13B (2.5, 5 and 10 uL of the IVT reaction) or insect cell purified GFP-ARL13B (2.5, 5 and $10 \mathrm{ug}$ ) in LAP100N (50 mM HEPES pH 7.4, 150 $\mathrm{mM} \mathrm{KCl}, 1 \mathrm{mM}$ EGTA, $1 \mathrm{mM} \mathrm{MgCl} 2,10 \%$ glycerol, $0.05 \% \mathrm{NP}-40$ ) or LAP $150 \mathrm{~N}$ buffer at $4^{\circ} \mathrm{C}$ or room temperature respectively for 1 hour. In figure $5 \mathrm{C}, 50$ ug of the indicated MBP proteins were added along with IVT Myc-ARL13B. Flowthroughs were collected, beads were washed, eluted by boiling in SDS sample buffer and subjected to western blotting.

\section{PIP and membrane lipid blots}

Binding of recombinant proteins to pre-spotted PIP and membrane lipid strips (Thermo Fisher; P23751) was performed according to (Dowler et al., 2002). After blocking strips in Licor blocking buffer for $1 \mathrm{hr}$ at room temperature, the strips were incubated in Licor blocking buffer containing $1 \mu \mathrm{g} / \mathrm{ml}$ of recombinant GST, GST-TULP3 or GST-TULP3 ${ }^{\mathrm{K} 3891}$ protein overnight at $4^{\circ} \mathrm{C}$. After washing three times in TBS-T, the blots were immunoblotted for GST.

\section{Mass spectrometry}

In the Taplin proteomics core $\left({ }^{\mathrm{LAP}} \mathrm{TULP} 3^{\mathrm{K} 3891}\right)$, excised gel bands were cut into approximately 1 $\mathrm{mm}^{3}$ pieces. For proximity biotinylation experiments, CD8-Gpr161 IC3 ${ }^{\text {WT }}$-BirA*, CD8-Gpr161 IC3 ${ }^{5 \mathrm{~A}}$-BirA*, CD8-MCHR1 IC3 ${ }^{\mathrm{WT}}$-BirA*, CD8-MCHR1 IC3 ${ }^{5 \mathrm{~A}}$-BirA* were co-transfected with LAPTULP3 in TREx-293 cells. After LAP tandem affinity purification, excised gel bands were submitted for post translational analysis at Taplin proteomics core for biotinylated peptides. Gel pieces were then subjected to a modified in-gel trypsin digestion procedure (Shevchenko et al., 1996). Gel pieces were washed and dehydrated with acetonitrile for 10 min followed by removal of acetonitrile. Pieces were then completely dried in a speed-vac. Rehydration of the gel pieces was with $50 \mathrm{mM}$ ammonium bicarbonate solution containing $12.5 \mathrm{ng} / \mu \mathrm{l}$ modified sequencinggrade trypsin (Promega, Madison, WI) at $4^{\circ} \mathrm{C}$. After $45 \mathrm{~min}$, the excess trypsin solution was removed and replaced with $50 \mathrm{mM}$ ammonium bicarbonate solution to just cover the gel pieces. Samples were then placed in a $37^{\circ} \mathrm{C}$ room overnight. Peptides were later extracted by 
removing the ammonium bicarbonate solution, followed by one wash with a solution containing $50 \%$ acetonitrile and $1 \%$ formic acid. The extracts were then dried in a speed-vac $(\sim 1 \mathrm{~h})$. The samples were then stored at $4^{\circ} \mathrm{C}$ until analysis. On the day of analysis, the samples were reconstituted in $5-10 \mu \mathrm{l}$ of HPLC solvent $\mathrm{A}$ ( $2.5 \%$ acetonitrile, $0.1 \%$ formic acid). A nano-scale reverse-phase HPLC capillary column was created by packing $2.6 \mu \mathrm{m} \mathrm{C} 18$ spherical silica beads into a fused silica capillary (100 $\mu \mathrm{m}$ inner diameter $\mathrm{x} \sim 30 \mathrm{~cm}$ length) with a flame-drawn tip (Peng and Gygi, 2001). After equilibrating the column each sample was loaded via a Famos auto sampler (LC Packings, San Francisco CA) onto the column. A gradient was formed, and peptides were eluted with increasing concentrations of solvent $B(97.5 \%$ acetonitrile, $0.1 \%$ formic acid). As peptides eluted, they were subjected to electrospray ionization and then entered an LTQ Orbitrap Velos Pro ion-trap mass spectrometer (Thermo Fisher Scientific, Waltham, MA). Peptides were detected, isolated, and fragmented to produce a tandem mass spectrum of specific fragment ions for each peptide. Peptide sequences (and hence protein identity) were determined by matching protein databases with the acquired fragmentation pattern by the software program, Sequest (Thermo Fisher Scientific, Waltham, MA) (Eng et al., 1994). Post translational analysis was performed using probability-based scoring (Beausoleil et al., 2006). All databases include a reversed version of all the sequences and the data was filtered to between a one and two percent peptide false discovery rate.

\section{Statistical Analyses}

Statistical analyses were performed using Student's $t$-test for comparing two groups or Tukey's post hoc multiple comparison tests between all possible pairs using GraphPad Prism. Nonparametric Matt Whiney-U test were performed for intensity plots using GraphPad Prism. 


\section{Acknowledgements}

This project was funded by PKD Foundation postdoctoral fellowship to VRP (214F19a), the National Institutes of Health (1R01GM113023 to S.M. and NS097928 to K.L.), a PKD Foundation grant to K. L. (232G18) and a Cancer Prevention and Research Institute of Texas grant (RR170063 to J.B.W), We thank molecular pathology and mass spectrometry cores and mouse animal care facility in UT Southwestern. We thank Ross Tomaino (Taplin mass spectrometry facility) for assistance with mass spectrometry. We acknowledge kind gifts of reagents from Tamara Caspary, Kathryn Anderson, David Beier, Peter Jackson, Peter Michaely, Takanari Inoue and Anne-Claude Gingras. We thank Issei Shimada and John Shelton for Nestin-Cre brain sections. We thank Sandii Constable for comments on the manuscript.

\section{Author contributions}

V.P. and S. M conceived the project, designed experiments, analyzed most of the data, and wrote the paper with inputs from all authors. S. H. generated cell lines and performed immunofluorescence experiments, B. N. S. performed kidney immunofluorescence experiments, H. B. generated mass spectrometry data on biotinylated samples, V. M.T. and J. B. W. provided help with insect cell purifications and reagents, E.L and K. L. generated Tulp3 mutant MEFs.

\section{Competing Financial Interest Statement}

The authors have no competing financial interests to declare. 


\section{References}

Antonny, B., S. Beraud-Dufour, P. Chardin, and M. Chabre. 1997. N-terminal hydrophobic residues of the G-protein ADP-ribosylation factor-1 insert into membrane phospholipids upon GDP to GTP exchange. Biochemistry. 36:4675-4684.

Anvarian, Z., K. Mykytyn, S. Mukhopadhyay, L.B. Pedersen, and S.T. Christensen. 2019. Cellular signalling by primary cilia in development, organ function and disease. Nat Rev Nephrol. 15:199-219.

Badgandi, H.B., S.H. Hwang, I.S. Shimada, E. Loriot, and S. Mukhopadhyay. 2017. Tubby family proteins are adapters for ciliary trafficking of integral membrane proteins. J Cell Biol. 216:743-760.

Barbeito, P., Y. Tachibana, R. Martin-Morales, P. Moreno, K. Mykytyn, T. Kobayashi, and F.R. Garcia-Gonzalo. 2020. Htr6 and Sstr3 ciliary targeting relies on both IC3 loops and Cterminal tails. bioRxiv:2020.2003.2019.997262.

Barral, D.C., S. Garg, C. Casalou, G.F. Watts, J.L. Sandoval, J.S. Ramalho, V.W. Hsu, and M.B. Brenner. 2012. Arl13b regulates endocytic recycling traffic. Proc Natl Acad Sci U S A. 109:21354-21359.

Beausoleil, S.A., J. Villen, S.A. Gerber, J. Rush, and S.P. Gygi. 2006. A probability-based approach for high-throughput protein phosphorylation analysis and site localization. Nat Biotechnol. 24:1285-1292.

Behal, R.H., M.S. Miller, H. Qin, B.F. Lucker, A. Jones, and D.G. Cole. 2012. Subunit interactions and organization of the Chlamydomonas reinhardtii intraflagellar transport complex $A$ proteins. J Biol Chem. 287:11689-11703.

Bennett, H.W., A.K. Gustavsson, C.A. Bayas, P.N. Petrov, N. Mooney, W.E. Moerner, and P.K. Jackson. 2020. Novel fibrillar structure in the inversin compartment of primary cilia revealed by 3D single-molecule superresolution microscopy. Mol Biol Cell. 31:619-639.

Bergmann, C., M. Fliegauf, N.O. Bruchle, V. Frank, H. Olbrich, J. Kirschner, B. Schermer, I. Schmedding, A. Kispert, B. Kranzlin, G. Nurnberg, C. Becker, T. Grimm, G. Girschick, S.A. Lynch, P. Kelehan, J. Senderek, T.J. Neuhaus, T. Stallmach, H. Zentgraf, P. Nurnberg, N. Gretz, C. Lo, S. Lienkamp, T. Schafer, G. Walz, T. Benzing, K. Zerres, and H. Omran. 2008. Loss of nephrocystin-3 function can cause embryonic lethality, Meckel-Gruber-like syndrome, situs inversus, and renal-hepatic-pancreatic dysplasia. Am J Hum Genet. 82:959-970.

Bielas, S.L., J.L. Silhavy, F. Brancati, M.V. Kisseleva, L. Al-Gazali, L. Sztriha, R.A. Bayoumi, M.S. Zaki, A. Abdel-Aleem, R.O. Rosti, H. Kayserili, D. Swistun, L.C. Scott, E. Bertini, E. Boltshauser, E. Fazzi, L. Travaglini, S.J. Field, S. Gayral, M. Jacoby, S. Schurmans, B. Dallapiccola, P.W. Majerus, E.M. Valente, and J.G. Gleeson. 2009. Mutations in INPP5E, encoding inositol polyphosphate-5-phosphatase $\mathrm{E}$, link phosphatidyl inositol signaling to the ciliopathies. Nat Genet. 41:1032-1036.

Cantagrel, V., J.L. Silhavy, S.L. Bielas, D. Swistun, S.E. Marsh, J.Y. Bertrand, S. Audollent, T. Attie-Bitach, K.R. Holden, W.B. Dobyns, D. Traver, L. Al-Gazali, B.R. Ali, T.H. Lindner, T. 
Caspary, E.A. Otto, F. Hildebrandt, I.A. Glass, C.V. Logan, C.A. Johnson, C. Bennett, F. Brancati, G. International Joubert Syndrome Related Disorders Study, E.M. Valente, C.G. Woods, and J.G. Gleeson. 2008. Mutations in the cilia gene ARL13B lead to the classical form of Joubert syndrome. Am J Hum Genet. 83:170-179.

Caspary, T., C.E. Larkins, and K.V. Anderson. 2007. The graded response to Sonic Hedgehog depends on cilia architecture. Dev Cell. 12:767-778.

Cevik, S., Y. Hori, O.I. Kaplan, K. Kida, T. Toivenon, C. Foley-Fisher, D. Cottell, T. Katada, K. Kontani, and O.E. Blacque. 2010. Joubert syndrome Arl13b functions at ciliary membranes and stabilizes protein transport in Caenorhabditis elegans. $J$ Cell Biol. 188:953-969.

Cevik, S., A.A. Sanders, E. Van Wijk, K. Boldt, L. Clarke, J. van Reeuwijk, Y. Hori, N. Horn, L. Hetterschijt, A. Wdowicz, A. Mullins, K. Kida, O.I. Kaplan, S.E. van Beersum, K. Man Wu, S.J. Letteboer, D.A. Mans, T. Katada, K. Kontani, M. Ueffing, R. Roepman, H. Kremer, and O.E. Blacque. 2013. Active transport and diffusion barriers restrict Joubert Syndromeassociated ARL13B/ARL-13 to an Inv-like ciliary membrane subdomain. PLoS Genet. 9:e1003977.

Chavez, M., S. Ena, J. Van Sande, A. de Kerchove d'Exaerde, S. Schurmans, and S.N. Schiffmann. 2015. Modulation of Ciliary Phosphoinositide Content Regulates Trafficking and Sonic Hedgehog Signaling Output. Dev Cell. 34:338-350.

Chih, B., P. Liu, Y. Chinn, C. Chalouni, L.G. Komuves, P.E. Hass, W. Sandoval, and A.S. Peterson. 2011. A ciliopathy complex at the transition zone protects the cilia as a privileged membrane domain. Nat Cell Biol. 14:61-72.

Dateyama, I., Y. Sugihara, S. Chiba, R. Ota, R. Nakagawa, T. Kobayashi, and H. Itoh. 2019. RABL2 positively controls localization of GPCRs in mammalian primary cilia. $J$ Cell Sci. 132.

Deretic, D., A.H. Williams, N. Ransom, V. Morel, P.A. Hargrave, and A. Arendt. 2005. Rhodopsin $\mathrm{C}$ terminus, the site of mutations causing retinal disease, regulates trafficking by binding to ADP-ribosylation factor 4 (ARF4). Proc Natl Acad Sci U S A. 102:3301-3306.

Donaldson, J.G., and C.L. Jackson. 2011. ARF family G proteins and their regulators: roles in membrane transport, development and disease. Nat Rev Mol Cell Biol. 12:362-375.

Dowler, S., G. Kular, and D.R. Alessi. 2002. Protein lipid overlay assay. Sci STKE. 2002:pl6.

Duldulao, N.A., S. Lee, and Z. Sun. 2009. Cilia localization is essential for in vivo functions of the Joubert syndrome protein Arl13b/Scorpion. Development. 136:4033-4042.

Eng, J.K., A.L. McCormack, and J.R. Yates. 1994. An approach to correlate tandem mass spectral data of peptides with amino acid sequences in a protein database. J Am Soc Mass Spectrom. 5:976-989.

Ferent, J., S. Constable, E.D. Gigante, P.T. Yam, L.E. Mariani, E. Legue, K.F. Liem, Jr., T. Caspary, and F. Charron. 2019. The Ciliary Protein Arl13b Functions Outside of the Primary Cilium in Shh-Mediated Axon Guidance. Cell Rep. 29:3356-3366 e3353. 
Follit, J.A., and G.J. Pazour. 2013. Analysis of ciliary membrane protein dynamics using SNAP technology. Methods Enzymol. 524:195-204.

Fu, W., L. Wang, S. Kim, J. Li, and B.D. Dynlacht. 2016. Role for the IFT-A Complex in Selective Transport to the Primary Cilium. Cell Rep. 17:1505-1517.

Garcia-Gonzalo, F.R., S.C. Phua, E.C. Roberson, G. Garcia, 3rd, M. Abedin, S. Schurmans, T. Inoue, and J.F. Reiter. 2015. Phosphoinositides Regulate Ciliary Protein Trafficking to Modulate Hedgehog Signaling. Dev Cell. 34:400-409.

Gautier, R., D. Douguet, B. Antonny, and G. Drin. 2008. HELIQUEST: a web server to screen sequences with specific alpha-helical properties. Bioinformatics. 24:2101-2102.

Geng, L., D. Okuhara, Z. Yu, X. Tian, Y. Cai, S. Shibazaki, and S. Somlo. 2006. Polycystin-2 traffics to cilia independently of polycystin-1 by using an N-terminal RVxP motif. J Cell Sci. 119:1383-1395.

Gigante, E.D., M.R. Taylor, A.A. Ivanova, R.A. Kahn, and T. Caspary. 2020. ARL13B regulates Sonic hedgehog signaling from outside primary cilia. Elife. 9.

Gotthardt, K., M. Lokaj, C. Koerner, N. Falk, A. Giessl, and A. Wittinghofer. 2015. A G-protein activation cascade from Arl13B to Arl3 and implications for ciliary targeting of lipidated proteins. Elife. 4.

Hakim, S., J.M. Dyson, S.J. Feeney, E.M. Davies, A. Sriratana, M.N. Koenig, O.V. Plotnikova, I.M. Smyth, S.D. Ricardo, R.M. Hobbs, and C.A. Mitchell. 2016. Inpp5e suppresses polycystic kidney disease via inhibition of PI3K/Akt-dependent mTORC1 signaling. Hum Mol Genet. 25:2295-2313.

Han, S., K. Miyoshi, S. Shikada, G. Amano, Y. Wang, T. Yoshimura, and T. Katayama. 2019. TULP3 is required for localization of membrane-associated proteins ARL13B and INPP5E to primary cilia. Biochem Biophys Res Commun. 509:227-234.

He, K., X. Ma, T. Xu, Y. Li, A. Hodge, Q. Zhang, J. Torline, Y. Huang, J. Zhao, K. Ling, and J. Hu. 2018. Axoneme polyglutamylation regulated by Joubert syndrome protein ARL13B controls ciliary targeting of signaling molecules. Nat Commun. 9:3310.

Hilgendorf, K.I., C.T. Johnson, and P.K. Jackson. 2016. The primary cilium as a cellular receiver: organizing ciliary GPCR signaling. Curr Opin Cell Biol. 39:84-92.

Hilgendorf, K.I., C.T. Johnson, A. Mezger, S.L. Rice, A.M. Norris, J. Demeter, W.J. Greenleaf, J.F. Reiter, D. Kopinke, and P.K. Jackson. 2019. Omega-3 Fatty Acids Activate Ciliary FFAR4 to Control Adipogenesis. Cell. 179:1289-1305 e1221.

Hirano, T., Y. Katoh, and K. Nakayama. 2017. Intraflagellar transport-A complex mediates ciliary entry and retrograde trafficking of ciliary $\mathrm{G}$ protein-coupled receptors. Mol Biol Cell. 28:429-439.

Hori, Y., T. Kobayashi, Y. Kikko, K. Kontani, and T. Katada. 2008. Domain architecture of the atypical Arf-family GTPase Arl13b involved in cilia formation. Biochem Biophys Res Commun. 373:119-124. 
Hou, X., M. Mrug, B.K. Yoder, E.J. Lefkowitz, G. Kremmidiotis, P. D'Eustachio, D.R. Beier, and L.M. Guay-Woodford. 2002. Cystin, a novel cilia-associated protein, is disrupted in the cpk mouse model of polycystic kidney disease. J Clin Invest. 109:533-540.

Humbert, M.C., K. Weihbrecht, C.C. Searby, Y. Li, R.M. Pope, V.C. Sheffield, and S. Seo. 2012. ARL13B, PDE6D, and CEP164 form a functional network for INPP5E ciliary targeting. Proc Natl Acad Sci U S A. 109:19691-19696.

Hwang, S.H., B.N. Somatilaka, H. Badgandi, V.R. Palicharla, R. Walker, J.M. Shelton, F. Qian, and S. Mukhopadhyay. 2019. Tulp3 Regulates Renal Cystogenesis by Trafficking of Cystoproteins to Cilia. Curr Biol. 29:790-802 e795.

Ivanova, A.A., T. Caspary, N.T. Seyfried, D.M. Duong, A.B. West, Z. Liu, and R.A. Kahn. 2017. Biochemical characterization of purified mammalian ARL13B protein indicates that is in atypical GTPase and ARL3 guanine nucleotide exchange factor (GEF). J Biol Chem. 292:11091-11108.

Jenkins, P.M., T.W. Hurd, L. Zhang, D.P. McEwen, R.L. Brown, B. Margolis, K.J. Verhey, and J.R. Martens. 2006. Ciliary targeting of olfactory CNG channels requires the CNGB1b subunit and the kinesin-2 motor protein, KIF17. Curr Biol. 16:1211-1216.

Keppler, A., S. Gendreizig, T. Gronemeyer, H. Pick, H. Vogel, and K. Johnsson. 2003. A general method for the covalent labeling of fusion proteins with small molecules in vivo. Nat Biotechnol. 21:86-89.

Kobayashi, T., Y. Ishida, T. Hirano, Y. Katoh, and K. Nakayama. 2021. Cooperation of the IFT-A complex with the IFT-B complex is required for ciliary retrograde protein trafficking and GPCR import. Mol Biol Cell. 32:45-56.

Kopinke, D., A.M. Norris, and S. Mukhopadhyay. 2020. Developmental and regenerative paradigms of cilia regulated hedgehog signaling. Semin Cell Dev Biol.

Larkins, C.E., G.D. Aviles, M.P. East, R.A. Kahn, and T. Caspary. 2011. Arl13b regulates ciliogenesis and the dynamic localization of Shh signaling proteins. Mol Biol Cell. 22:46944703.

Legue, E., and K.F. Liem, Jr. 2019. Tulp3 Is a Ciliary Trafficking Gene that Regulates Polycystic Kidney Disease. Curr Biol. 29:803-812 e805.

Legue, E., and K.F. Liem, Jr. 2020. Mutations in Ciliary Trafficking Genes affect Sonic Hedgehogdependent Neural Tube Patterning Differentially along the Anterior-Posterior Axis. Neuroscience. 450:3-14.

Lemaitre, R.P., A. Bogdanova, B. Borgonovo, J.B. Woodruff, and D.N. Drechsel. 2019. FlexiBAC: a versatile, open-source baculovirus vector system for protein expression, secretion, and proteolytic processing. BMC Biotechnol. 19:20.

Li, Y., X. Tian, M. Ma, S. Jerman, S. Kong, S. Somlo, and Z. Sun. 2016. Deletion of ADP Ribosylation Factor-Like GTPase 13B Leads to Kidney Cysts. J Am Soc Nephrol. 27:36283638. 
Liem, K.F., Jr., A. Ashe, M. He, P. Satir, J. Moran, D. Beier, C. Wicking, and K.V. Anderson. 2012. The IFT-A complex regulates Shh signaling through cilia structure and membrane protein trafficking. J Cell Biol. 197:789-800.

Loktev, A.V., and P.K. Jackson. 2013. Neuropeptide $Y$ family receptors traffic via the Bardet-Biedl syndrome pathway to signal in neuronal primary cilia. Cell Rep. 5:1316-1329.

Lu, H., M.T. Toh, V. Narasimhan, S.K. Thamilselvam, S.P. Choksi, and S. Roy. 2015. A function for the Joubert syndrome protein Arl13b in ciliary membrane extension and ciliary length regulation. Dev Biol. 397:225-236.

Ma, M. 2021. Cilia and polycystic kidney disease. Semin Cell Dev Biol. 110:139-148.

Mariani, L.E., M.F. Bijlsma, A.A. Ivanova, S.K. Suciu, R.A. Kahn, and T. Caspary. 2016. Arl13b regulates Shh signaling from both inside and outside the cilium. Mol Biol Cell.

Mick, D.U., R.B. Rodrigues, R.D. Leib, C.M. Adams, A.S. Chien, S.P. Gygi, and M.V. Nachury. 2015. Proteomics of Primary Cilia by Proximity Labeling. Dev Cell. 35:497-512.

Miertzschke, M., C. Koerner, M. Spoerner, and A. Wittinghofer. 2014. Structural insights into the small G-protein Arl13B and implications for Joubert syndrome. Biochem J. 457:301-311.

Mouillac, B., M. Caron, H. Bonin, M. Dennis, and M. Bouvier. 1992. Agonist-modulated palmitoylation of beta 2-adrenergic receptor in Sf9 cells. J Biol Chem. 267:21733-21737.

Mukhopadhyay, S., H.B. Badgandi, S.H. Hwang, B. Somatilaka, I.S. Shimada, and K. Pal. 2017. Trafficking to the primary cilium membrane. Mol Biol Cell. 28:233-239.

Mukhopadhyay, S., and P.K. Jackson. 2011. The tubby family proteins. Genome Biology. 12:225.

Mukhopadhyay, S., X. Wen, B. Chih, C.D. Nelson, W.S. Lane, S.J. Scales, and P.K. Jackson. 2010. TULP3 bridges the IFT-A complex and membrane phosphoinositides to promote trafficking of G protein-coupled receptors into primary cilia. Genes Dev. 24:2180-2193.

Mukhopadhyay, S., X. Wen, N. Ratti, A. Loktev, L. Rangell, S.J. Scales, and P.K. Jackson. 2013. The ciliary G-protein-coupled receptor Gpr161 negatively regulates the Sonic hedgehog pathway via cAMP signaling. Cell. 152:210-223.

Nachury, M.V., and D.U. Mick. 2019. Establishing and regulating the composition of cilia for signal transduction. Nat Rev Mol Cell Biol. 20:389-405.

Ng, G.Y., B. Mouillac, S.R. George, M. Caron, M. Dennis, M. Bouvier, and B.F. O'Dowd. 1994. Desensitization, phosphorylation and palmitoylation of the human dopamine D1 receptor. Eur J Pharmacol. 267:7-19.

Norman, R.X., H.W. Ko, V. Huang, C.M. Eun, L.L. Abler, Z. Zhang, X. Sun, and J.T. Eggenschwiler. 2009. Tubby-like protein 3 (TULP3) regulates patterning in the mouse embryo through inhibition of Hedgehog signaling. Hum Mol Genet. 18:1740-1754. 
Nozaki, S., Y. Katoh, M. Terada, S. Michisaka, T. Funabashi, S. Takahashi, K. Kontani, and K. Nakayama. 2017. Regulation of ciliary retrograde protein trafficking by the Joubert syndrome proteins ARL13B and INPP5E. J Cell Sci. 130:563-576.

Ocbina, P.J., and K.V. Anderson. 2008. Intraflagellar transport, cilia, and mammalian Hedgehog signaling: analysis in mouse embryonic fibroblasts. Dev Dyn. 237:2030-2038.

Peng, J., and S.P. Gygi. 2001. Proteomics: the move to mixtures. J Mass Spectrom. 36:10831091.

Picariello, T., J.M. Brown, Y. Hou, G. Swank, D.A. Cochran, O.D. King, K. Lechtreck, G.J. Pazour, and G.B. Witman. 2019. A global analysis of IFT-A function reveals specialization for transport of membrane-associated proteins into cilia. J Cell Sci.

Qin, J., Y. Lin, R.X. Norman, H.W. Ko, and J.T. Eggenschwiler. 2011. Intraflagellar transport protein 122 antagonizes Sonic Hedgehog signaling and controls ciliary localization of pathway components. Proc Natl Acad Sci U S A. 108:1456-1461.

Qiu, H., S. Fujisawa, S. Nozaki, Y. Katoh, and K. Nakayama. 2021. Interaction of INPP5E with ARL13B is essential for its ciliary membrane retention but dispensable for its ciliary entry. Biol Open. 10.

Randazzo, P.A., T. Terui, S. Sturch, H.M. Fales, A.G. Ferrige, and R.A. Kahn. 1995. The myristoylated amino terminus of ADP-ribosylation factor 1 is a phospholipid- and GTPsensitive switch. J Biol Chem. 270:14809-14815.

Ren, J., L. Wen, X. Gao, C. Jin, Y. Xue, and X. Yao. 2008. CSS-Palm 2.0: an updated software for palmitoylation sites prediction. Protein Eng Des Sel. 21:639-644.

Roux, K.J., D.I. Kim, M. Raida, and B. Burke. 2012. A promiscuous biotin ligase fusion protein identifies proximal and interacting proteins in mammalian cells. The Journal of cell biology. 196:801-810.

Santagata, S., T.J. Boggon, C.L. Baird, C.A. Gomez, J. Zhao, W.S. Shan, D.G. Myszka, and L. Shapiro. 2001. G-protein signaling through tubby proteins. Science. 292:2041-2050.

Seixas, C., S.Y. Choi, N. Polgar, N.L. Umberger, M.P. East, X. Zuo, H. Moreiras, R. Ghossoub, A. Benmerah, R.A. Kahn, B. Fogelgren, T. Caspary, J.H. Lipschutz, and D.C. Barral. 2016. Arl13b and the exocyst interact synergistically in ciliogenesis. Mol Biol Cell. 27:308-320.

Shao, X., S. Somlo, and P. Igarashi. 2002. Epithelial-specific Cre/lox recombination in the developing kidney and genitourinary tract. J Am Soc Nephrol. 13:1837-1846.

Shevchenko, A., M. Wilm, O. Vorm, and M. Mann. 1996. Mass spectrometric sequencing of proteins silver-stained polyacrylamide gels. Anal Chem. 68:850-858.

Shiba, D., D.K. Manning, H. Koga, D.R. Beier, and T. Yokoyama. 2010. Inv acts as a molecular anchor for Nphp3 and Nek8 in the proximal segment of primary cilia. Cytoskeleton (Hoboken). 67:112-119. 
Stolc, V., M.P. Samanta, W. Tongprasit, and W.F. Marshall. 2005. Genome-wide transcriptional analysis of flagellar regeneration in Chlamydomonas reinhardtii identifies orthologs of ciliary disease genes. Proc Natl Acad Sci U S A. 102:3703-3707.

Su, S., S.C. Phua, R. DeRose, S. Chiba, K. Narita, P.N. Kalugin, T. Katada, K. Kontani, S. Takeda, and T. Inoue. 2013. Genetically encoded calcium indicator illuminates calcium dynamics in primary cilia. Nat Methods. 10:1105-1107.

Sun, X., J. Haley, O.V. Bulgakov, X. Cai, J. McGinnis, and T. Li. 2012. Tubby is required for trafficking $G$ protein-coupled receptors to neuronal cilia. Cilia. 1:21.

Sun, Z., A. Amsterdam, G.J. Pazour, D.G. Cole, M.S. Miller, and N. Hopkins. 2004. A genetic screen in zebrafish identifies cilia genes as a principal cause of cystic kidney. Development. 131:4085-4093.

Svendsen, S., C. Zimprich, M.G. McDougall, D.H. Klaubert, and G.V. Los. 2008. Spatial separation and bidirectional trafficking of proteins using a multi-functional reporter. $B M C$ Cell Biol. 9:17.

Takahara, M., Y. Katoh, K. Nakamura, T. Hirano, M. Sugawa, Y. Tsurumi, and K. Nakayama. 2018. Ciliopathy-associated mutations of IFT122 impair ciliary protein trafficking but not ciliogenesis. Hum Mol Genet. 27:516-528.

Thomas, S., V. Cantagrel, L. Mariani, V. Serre, J.E. Lee, N. Elkhartoufi, P. de Lonlay, I. Desguerre, A. Munnich, N. Boddaert, S. Lyonnet, M. Vekemans, S.N. Lisgo, T. Caspary, J. Gleeson, and T. Attie-Bitach. 2015. Identification of a novel ARL13B variant in a Joubert syndromeaffected patient with retinal impairment and obesity. Eur J Hum Genet. 23:621-627.

Torres, J.Z., J.J. Miller, and P.K. Jackson. 2009. High-throughput generation of tagged stable cell lines for proteomic analysis. Proteomics. 9:2888-2891.

Tran, P.V., C.J. Haycraft, T.Y. Besschetnova, A. Turbe-Doan, R.W. Stottmann, B.J. Herron, A.L. Chesebro, H. Qiu, P.J. Scherz, J.V. Shah, B.K. Yoder, and D.R. Beier. 2008. THM1 negatively modulates mouse sonic hedgehog signal transduction and affects retrograde intraflagellar transport in cilia. Nat Genet. 40:403-410.

Tronche, F., C. Kellendonk, O. Kretz, P. Gass, K. Anlag, P.C. Orban, R. Bock, R. Klein, and G. Schutz. 1999. Disruption of the glucocorticoid receptor gene in the nervous system results in reduced anxiety. Nat Genet. 23:99-103.

VanderWaal Mills, K.E. 2011. Flagellar proteins regulating motility, assembly and photobehavior in Chlamydomonas reinhardtii. , Retrieved from the University of Minnesota Digital Conservancy.

Viau, A., F. Bienaime, K. Lukas, A.P. Todkar, M. Knoll, T.A. Yakulov, A. Hofherr, O. Kretz, M. Helmstadter, W. Reichardt, S. Braeg, T. Aschman, A. Merkle, D. Pfeifer, V.I. Dumit, M.C. Gubler, R. Nitschke, T.B. Huber, F. Terzi, J. Dengjel, F. Grahammer, M. Kottgen, H. Busch, M. Boerries, G. Walz, A. Triantafyllopoulou, and E.W. Kuehn. 2018. Cilia-localized LKB1 regulates chemokine signaling, macrophage recruitment, and tissue homeostasis in the kidney. EMBO J. 37. 
Wang, J., Y. Morita, J. Mazelova, and D. Deretic. 2012. The Arf GAP ASAP1 provides a platform to regulate Arf4- and Rab11-Rab8-mediated ciliary receptor targeting. EMBO J. 31:40574071.

Wang, W., L.M. Silva, B.A. Allard, T.S. Pottorf, H.H. Wang, D.T. Jacobs, J.T. Cornelius, A. Chaturvedi, M.T. Pritchard, M. Sharma, D.P. Wallace, J.P. Calvet, and P.V. Tran. 2020. Intraflagellar transport-A deficiency ameliorates ADPKD renal cystogenesis in a renal tubular- and maturation-dependent manner. bioRxiv:2020.2004.2026.061796.

Ward, H.H., U. Brown-Glaberman, J. Wang, Y. Morita, S.L. Alper, E.J. Bedrick, V.H. Gattone, 2nd, D. Deretic, and A. Wandinger-Ness. 2011. A conserved signal and GTPase complex are required for the ciliary transport of polycystin-1. Mol Biol Cell. 22:3289-3305.

Weng, S.L., H.J. Kao, C.H. Huang, and T.Y. Lee. 2017. MDD-Palm: Identification of protein Spalmitoylation sites with substrate motifs based on maximal dependence decomposition. PLoS One. 12:e0179529.

Wright, K.J., L.M. Baye, A. Olivier-Mason, S. Mukhopadhyay, L. Sang, M. Kwong, W. Wang, P.R. Pretorius, V.C. Sheffield, P. Sengupta, D.C. Slusarski, and P.K. Jackson. 2011. An ARL3UNC119-RP2 GTPase cycle targets myristoylated NPHP3 to the primary cilium. Genes \& development. 25:2347-2360.

Wu, C.-T., K.I. Hilgendorf, R.J. Bevacqua, Y. Hang, J. Demeter, S.K. Kim, and P.K. Jackson. 2020. Discovery of ciliary $G$ protein-coupled receptors regulating pancreatic islet insulin and glucagon secretion. bioRxiv:2020.2010.2021.349423.

Yu, J., T.J. Carroll, and A.P. McMahon. 2002. Sonic hedgehog regulates proliferation and differentiation of mesenchymal cells in the mouse metanephric kidney. Development. 129:5301-5312.

Zeqiraj, E., B.M. Filippi, M. Deak, D.R. Alessi, and D.M. van Aalten. 2009. Structure of the LKB1STRAD-MO25 complex reveals an allosteric mechanism of kinase activation. Science. 326:1707-1711. 
Figure 1. Tulp3 determines ciliary trafficking of Arl13b.

(A) MEFs from wild type or Tulp3 knockout (ko) mice were serum starved upon confluence for 24 $\mathrm{h}$ before fixation. Fixed cells were immunostained for Arl13b (green) or Gpr161 (shown in Figure S1) along with acetylated tubulin (AcTub, red) and counterstained for DNA (blue).

(B) Arl13b (from A) or Gpr161 (from Figure S1) positive cilia were counted from two experiments, and total counted cells are $>200$ for each condition. Data represent mean \pm SD.

(C) Cilia length was measured from wild type or Tulp3 ko MEFs by immunostaining for acetylated tubulin. Number of cilia measured for length is $>100$ for each condition.

(D) Wildtype and Tulp3 ko 3T3-L1 preadipocyte cells grown to confluency and cultured further for $72 \mathrm{~h}$ to promote ciliation before fixing. The fixed cells were immunostained for Arl13b (green) along with acetylated tubulin (red) and counterstained for DNA.

(E) Arl13b positive cilia from D were counted from three experiments, and total counted cells are $>200$ for each condition. Data represent mean \pm SD. Inset shows immunoblot of wild type or Tulp3 ko 3T3L1 cell lysates with Tulp3 and Tubulin antibodies.

(F) Cilia length was measured from wild type or Tulp3 ko 3T3L1 cells by by immunostaining for acetylated tubulin. $n>50$ for each condition.

(G) Brain sections from caudate-putamen $(\mathrm{CPu})$ and $\mathrm{CA} 1$ regions in hippocampus from either control or Nestin-Cre; Tulp3 $3^{\text {f/f }}$ mice at P7 were immunostained for Arl13b and AC3 and counterstained for DNA.

(H) Arl13b positive cilia similar to (G) were counted from control or conditional knockout (cko) at P7. Total counted cilia are $>400$ for caudate-putamen regions, 50-250 for CA1 regions, and $>650$ for cerebellums for each mouse from one control and two cko mice. Data represent mean \pm SD.

(I) Tulp3 transcript levels relative to Gapdh were measured from control or Nestin Cre; Tulp3 ${ }^{\mathrm{f} / \mathrm{f}}$ mice. Data represents mean \pm SEM from two data points from one mouse.

(J) Wild type and Tulp3 ko IMCD3 cells stably expressing GFP-SNAPARL13B were starved for a total of $36 \mathrm{~h}$ after reaching confluence. After blocking intracellular pools of GFP-SNAPARL13B using BG-Block, cells were washed and later fixed at indicated times. "Block" refers to cells immediately fixed after blocking. Control refers to cells untreated with BG-block. Newly trafficked SNAP-tagged proteins in cilia in BG-block treated cells were tracked by immunofluorescence using fluorescent SNAP substrate (TMR-Star) and acetylated tubulin along with counterstaining for DNA. Images in Figure S1C. Violin plots of ciliary intensities of TMR-Star from $\mathrm{n}>30$ cells for each condition.

Scale, $5 \mu \mathrm{m} ;{ }^{* * *}, \mathrm{p}<0.0001 ;{ }^{* * *}, \mathrm{p}<0.001 ;{ }^{* *}, \mathrm{p}<0.01 ; \mathrm{ns}$, not significant. Arrows indicate cilia positive for the indicated proteins while arrowheads indicate negative cilia. See also Figure S1 and Figure S2. 
bioRxiv preprint doi: https://doi.org/10.1101/2021.05.25.445488; this version posted May 25, 2021. The copyright hpler for this preprint (w/ich
was not certified by peer review) is the author/funder. All rights reserved. No reuse allowed without pe 1

A

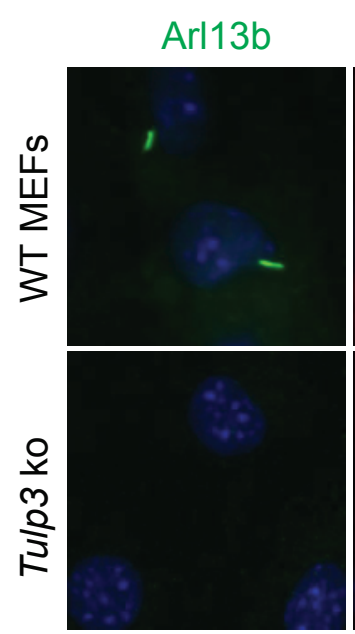

D
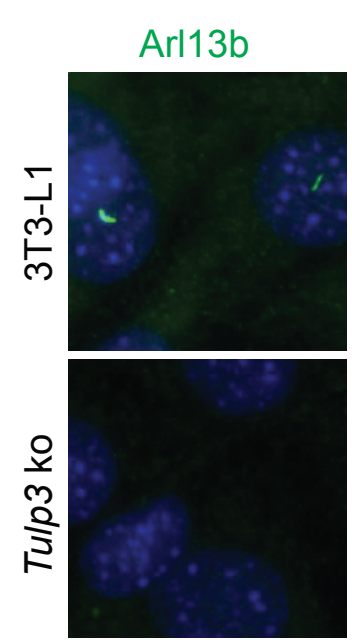

G

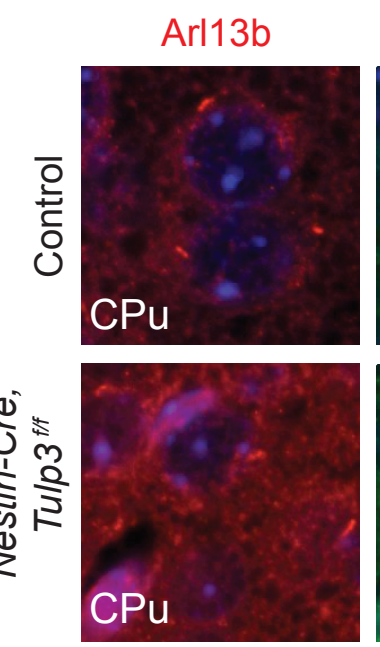

Starved at Intracellular-

confluence block

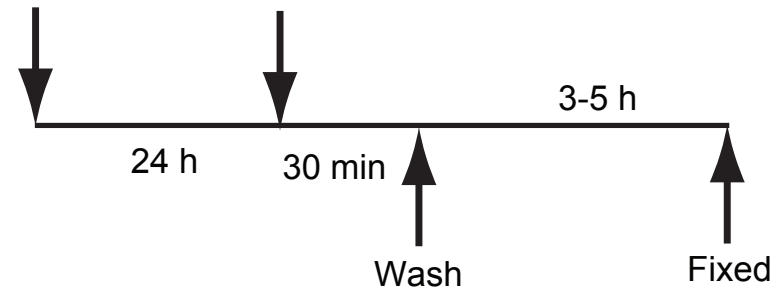

IF with intracellular SNAP-substrate

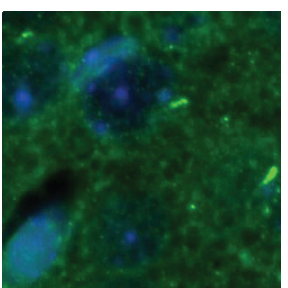

B
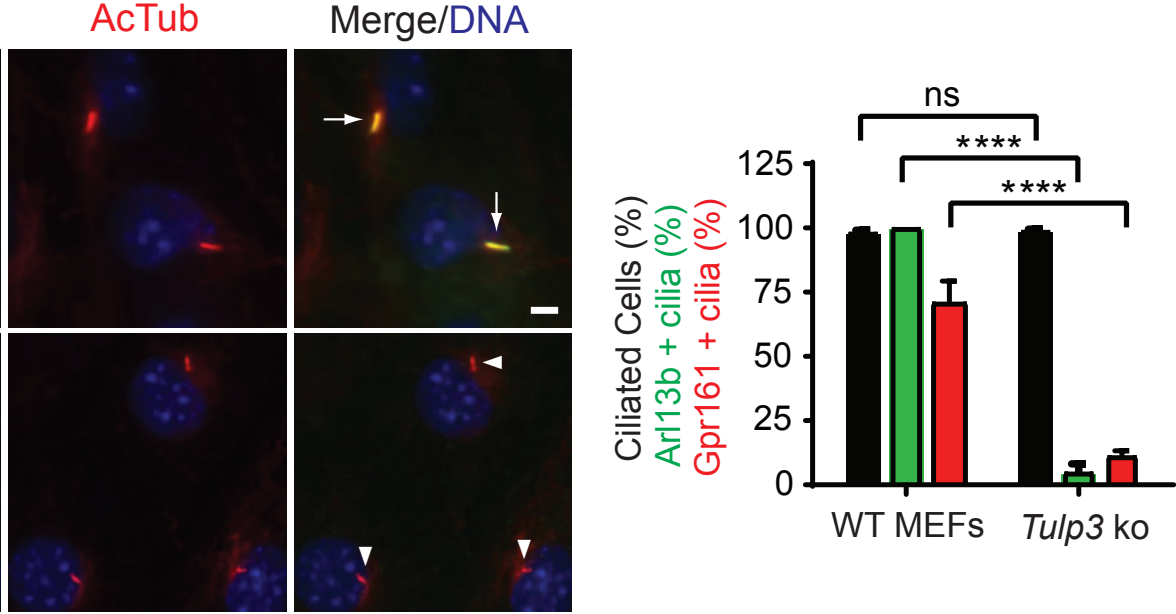

F

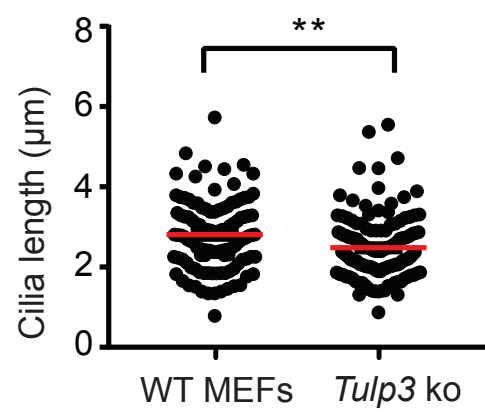

$E$
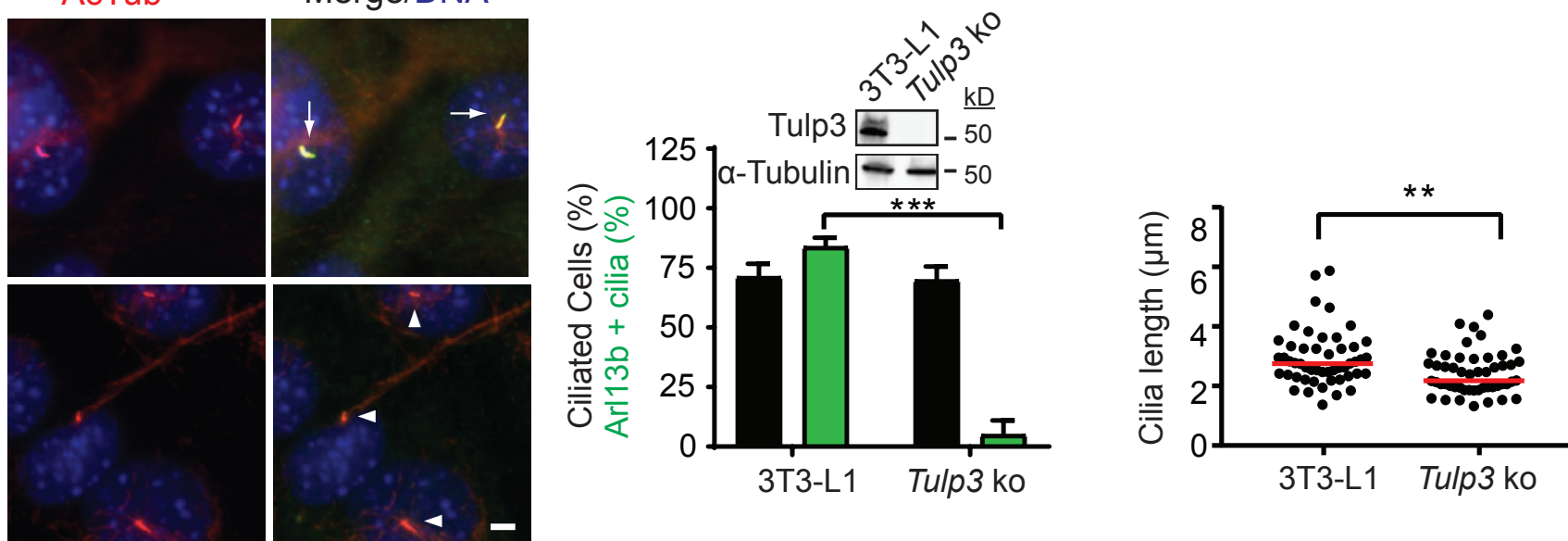

$\mathrm{H}$
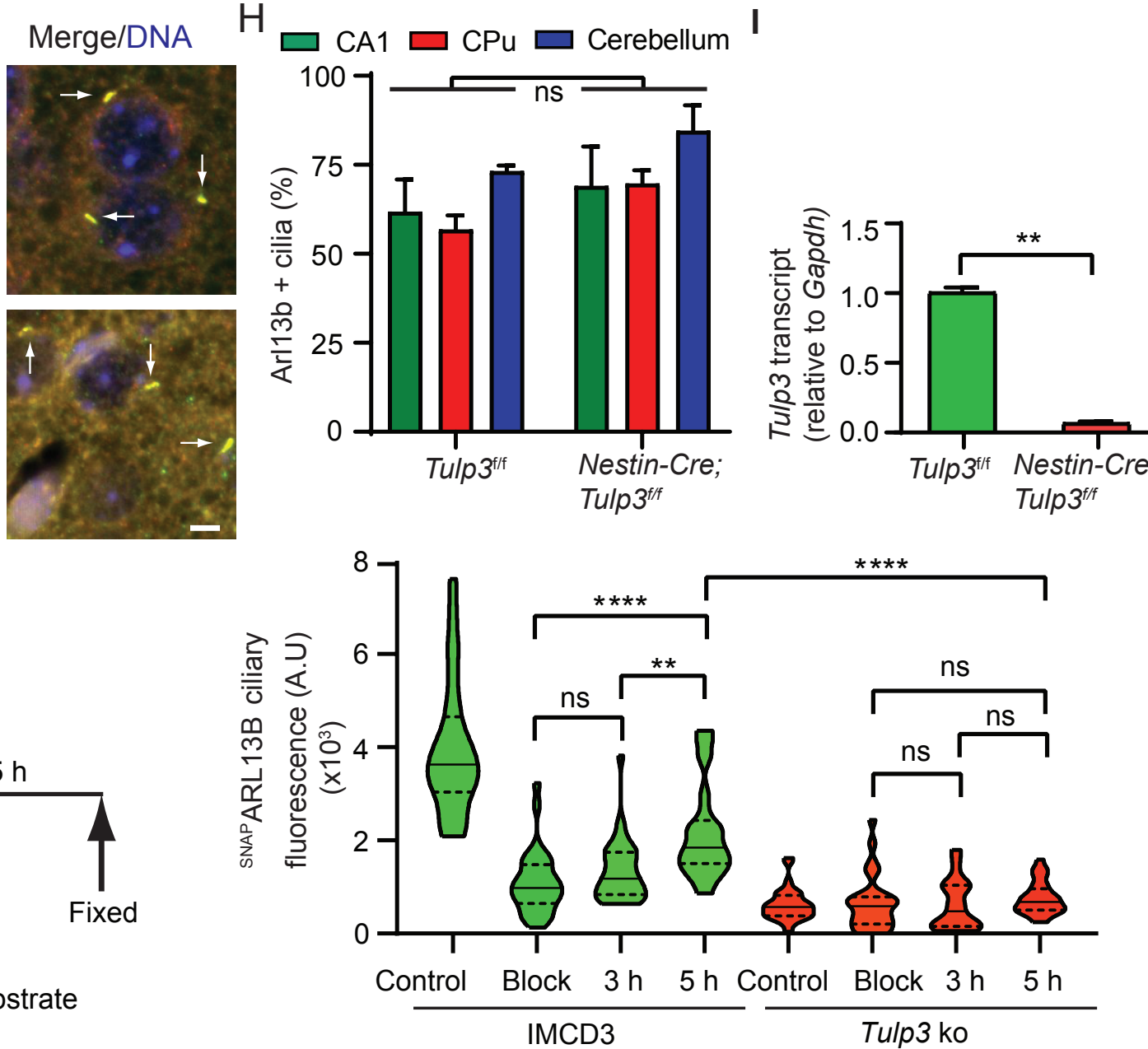
Figure 2. Tulp3 determines ciliary trafficking of Arl13b dependent cargoes.

(A) Cartoon depicting the role of Arl13b in regulating the release of farnesylated and myristoylated cargoes in the ciliary compartment via Arl3 (see text). Also shows a list of lipidated proteins that are known to localize to cilia.

(B) Wild type and Tulp3 ko IMCD3 cells (Figure S1B) stably expressing LAPINPP5E $\pm{ }^{\text {Myc }}$ TULP3 were grown till confluence and serum starved for 36 hours before fixation. Fixed cells were immunostained for endogenous Arl13b, GFP (INPP5E), acetylated tubulin (AcTub) and counterstained for DNA.

(C) Arl13b and GFP positive cilia from (B) were counted from two experiments and >200 cilia were counted for each condition. Data represent means \pm SD. Western blot shows Tulp3, Arl13b and $\alpha$ Tubulin protein levels from wild type and Tulp3 ko cells.

(D) Wild type or Tulp3 ko IMCD3 cells stably expressing NPHP3 ${ }^{\text {LAP }}$ or CYS1 ${ }^{\text {LAP }}$ were grown till confluence and serum starved for 36 hours before fixation. Fixed cells were immunostained for GFP along with acetylated tubulin (Actub) and counterstained for DNA.

(E) GFP positive cilia from (D) were counted from two experiments, and total counted cilia are $>200$ for each condition. Data represent mean \pm SD.

(F) Mean GFP ciliary fluorescence intensities were measured from NPHP3 ${ }^{\mathrm{LAP}}$ or Cys $1^{\mathrm{LAP}}$ expressing wild type or Tulp3 ko IMCD3 cells and shown as a violin plot. $>30$ cilia per condition were measured.

(G) Wild type or Tulp3 ko IMCD3 cells were grown till confluence and serum starved for 36 hours before fixation. Fixed cells were immunostained for Lkb1 along with acetylated tubulin (Actub) and counterstained for DNA.

(H) Lkb1 positive cilia from (G) were counted from two experiments, and total counted cilia are $>200$ for each condition. Data represent mean \pm SD.

(I) Mean Lkb1 ciliary fluorescence intensities were measured from wild type or Tulp3 ko IMCD3 cells. and shown as a violin plot. $>30$ cilia per condition were measured.

(J) Cilia lengths were measured from indicated cells as described in (B) by acetylated tubulin immunostaining (50 cilia per condition).

Scale, $5 \mu \mathrm{m} .{ }^{* * * *}, p<0.0001 ;{ }^{* * *}, p<0.001 ;{ }^{* *}, p<0.01 ;{ }^{*}, p<0.05$; Arrows indicate cilia positive for the indicated proteins while arrow heads indicate negative cilia. Yellow arrows point to cilia with low intensity of fluorescence. See also Figure S3. 
A

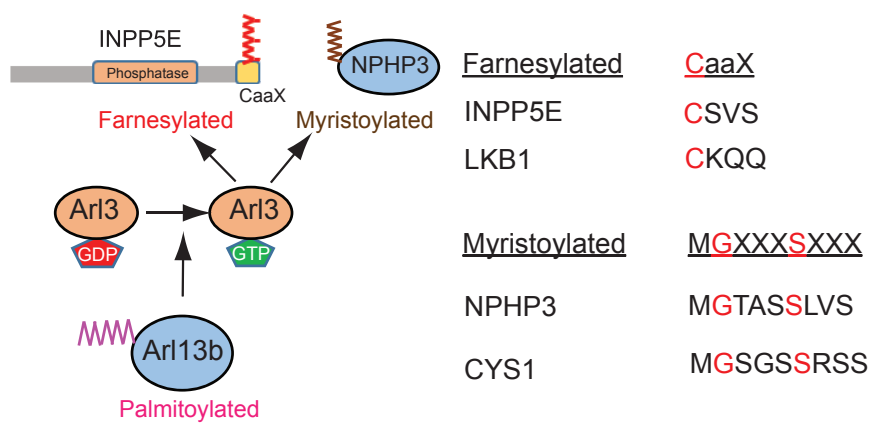

C

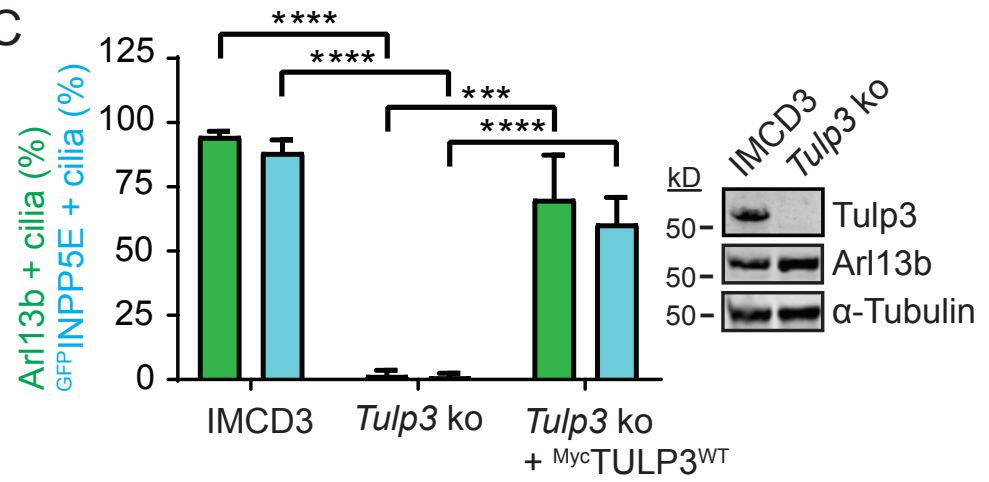

$\mathrm{E}$
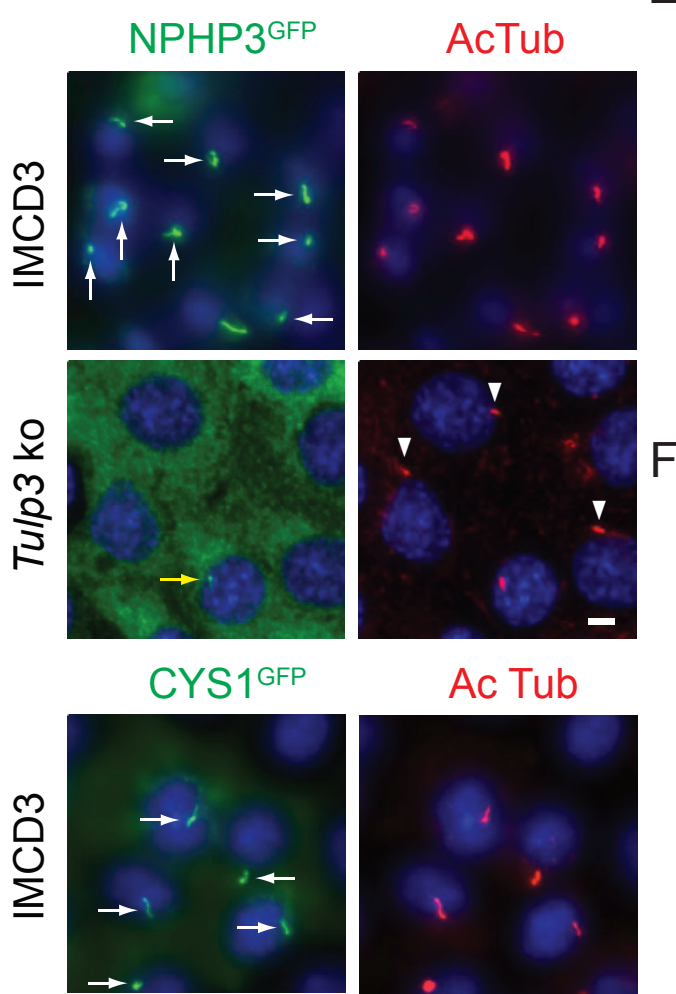

$\frac{1}{m}$

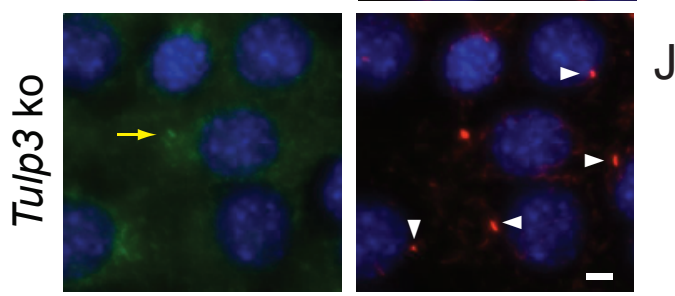

AcTub

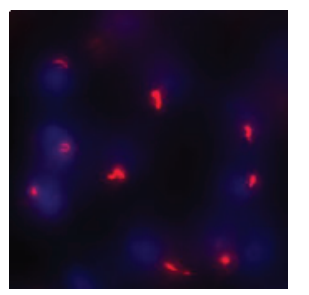

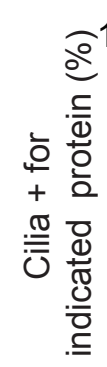
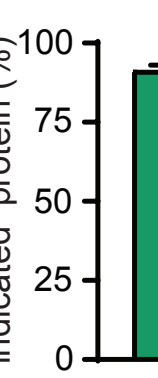

$\mathrm{F}$

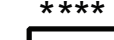

B

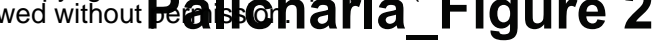

. No reuse allowed withou trealion
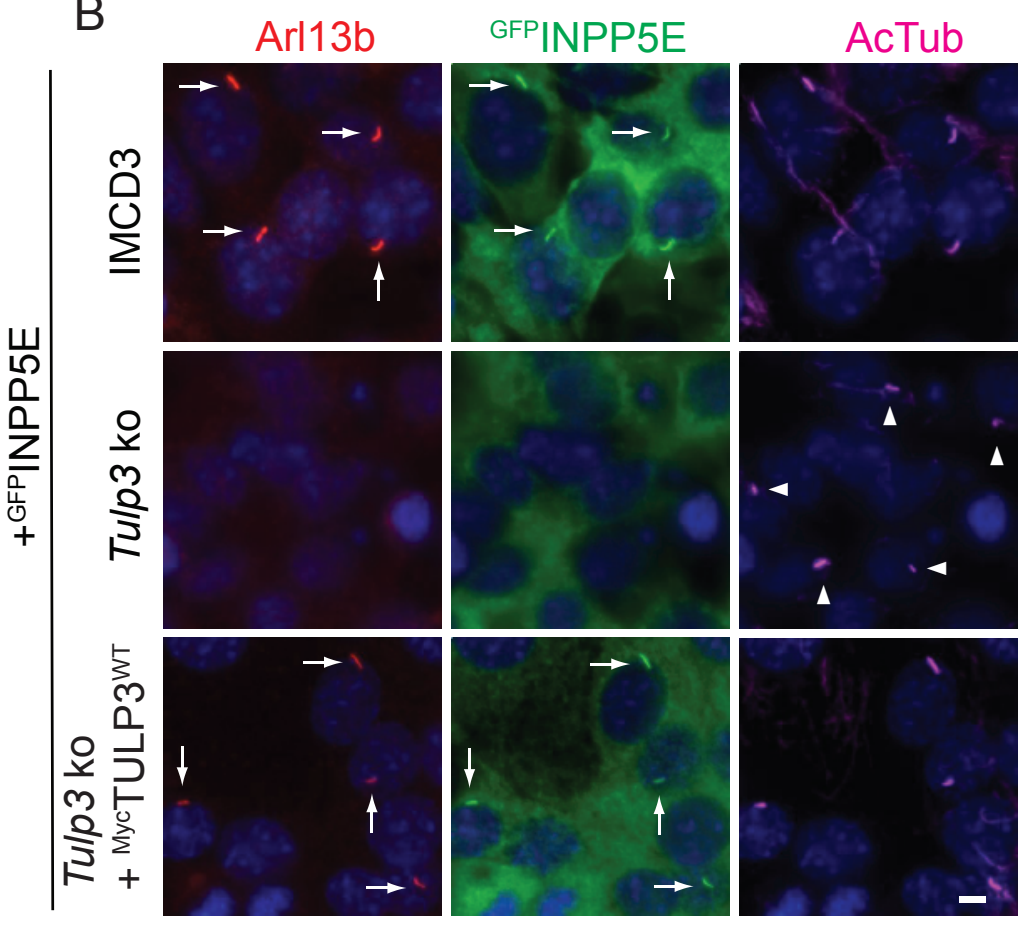
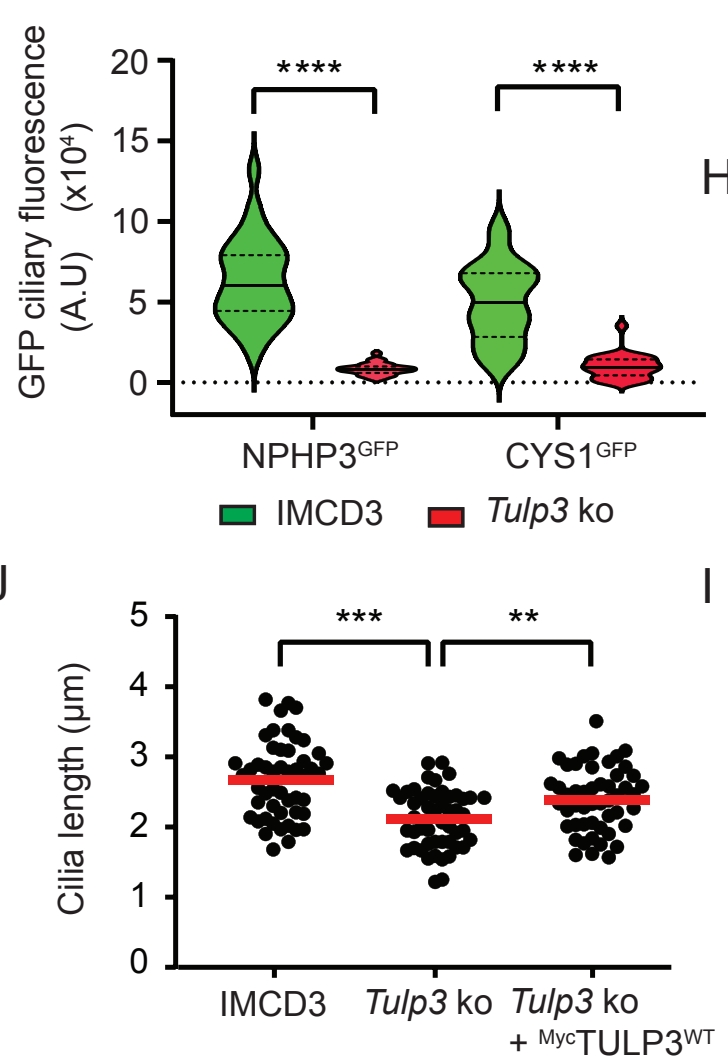

G
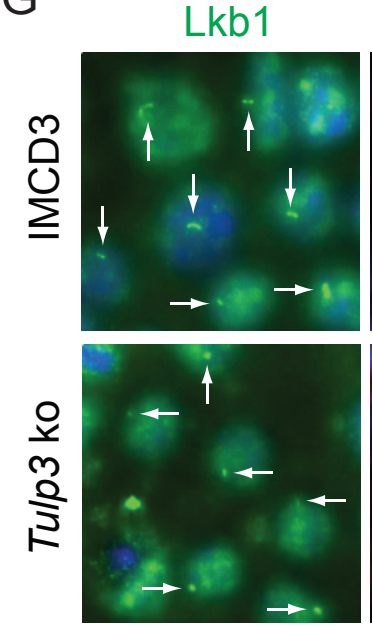

AcTub

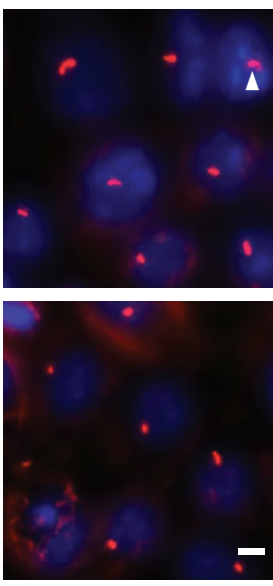

H
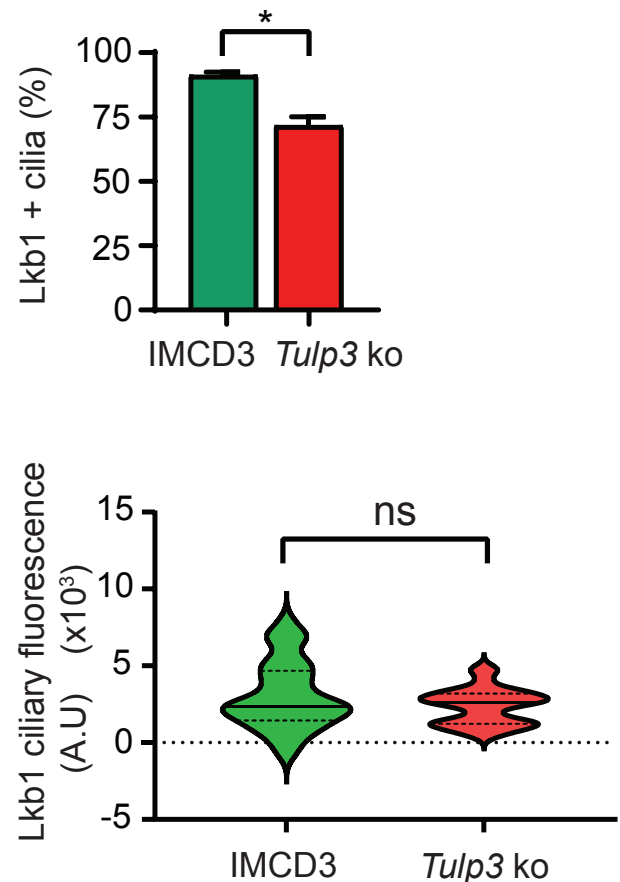
Figure 3. Tulp3 is required for trafficking of Arl13b-dependent lipidated cargoes to mouse kidney epithelial cilia.

(A-B) Kidney sagittal sections from either control, HoxB7-Cre; Tulp3 $3^{\mathrm{fff}}$ or Ksp-Cre; Tulp3 $3^{\mathrm{f} / \mathrm{f}}$ animals at postnatal days 0,5 and 24 were immunostained for Nphp3 (A) or Lkb1 (B), acetylated tubulin (AcTub) and Aquaporin 2 and counterstained for DNA.

(C-D) Percent positive cilia for the indicated proteins determined by immunostaining as in (A-B) were counted at postnatal days 0, 5 and 24 from 2-4 mice each genotype. Cilia counted by AcTub staining. $>200$ cilia were counted per mouse per genotype. Data represent mean \pm SD.

Scale, $5 \mu \mathrm{m}$. ${ }^{* * *}, \mathrm{p}<0.0001 ;{ }^{* *}, \mathrm{p}<0.01 ;{ }^{*}, \mathrm{p}<0.05$; ns, not significant. Arrows indicate cilia positive for the indicated proteins while arrow heads indicate negative cilia. 

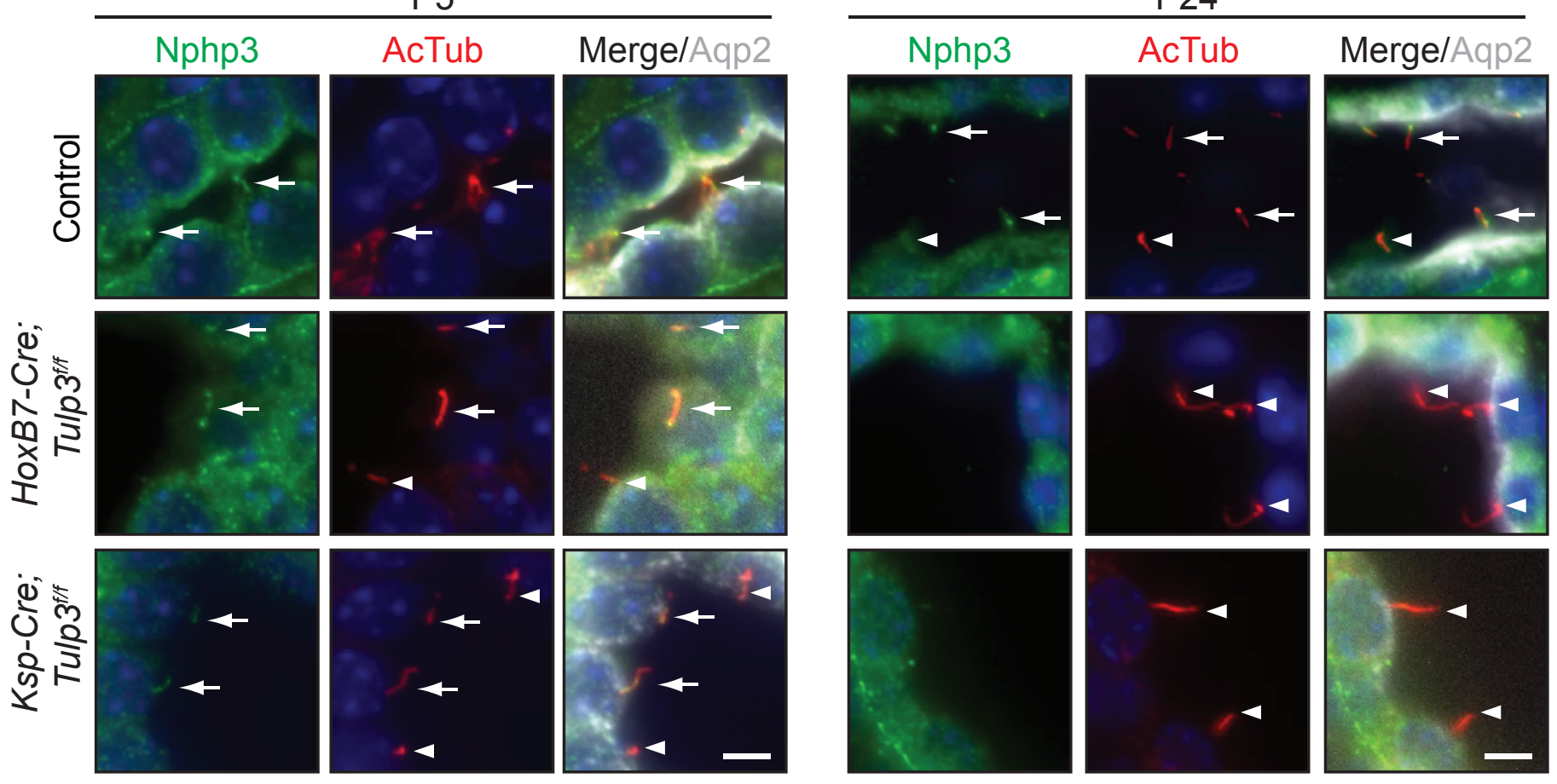

B
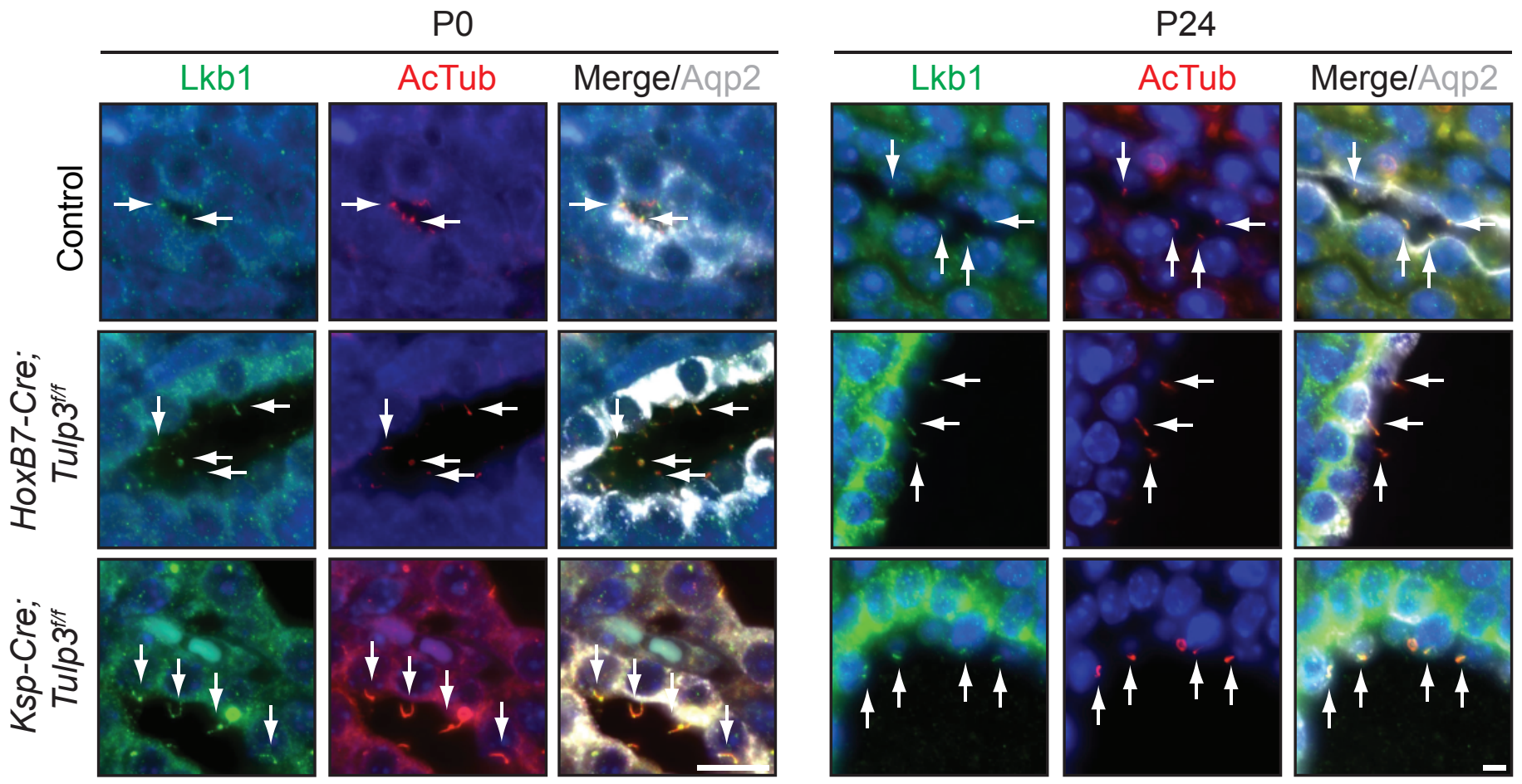

C

$\multimap$ Arl13b $\rightarrow$ Inpp5e $\rightarrow$ Nphp3 $\rightarrow$ Lkb1

D

$\rightarrow$ Control

$\rightarrow$ - HoxB7-Cre; Tulp3

$\leftarrow$ Ksp-Cre; Tulp3 $3^{\text {ft }}$

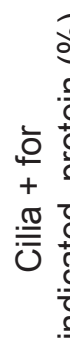

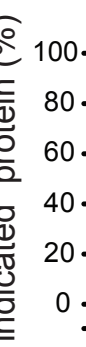

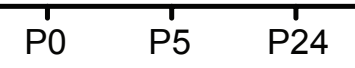

Control

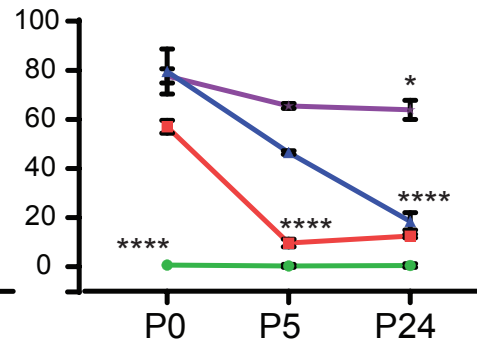

HoxB7-Cre; Tulp3 $3^{\text {fff }}$

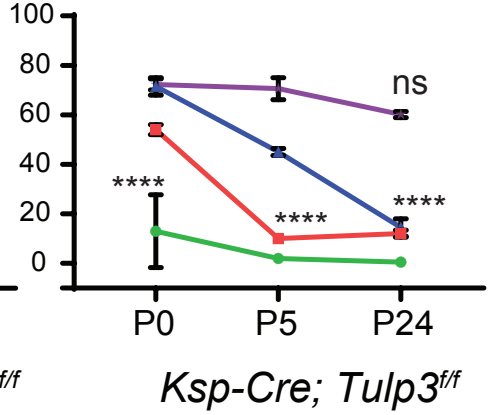


Figure 4. Tulp3 traffics Arl13b-dependent lipidated cargoes in a $\mathrm{PI}(4,5) \mathrm{P}_{2}$ independent manner unlike transmembrane GPCR cargoes.

(A) Schematic showing the nature of different mutants used in (B).

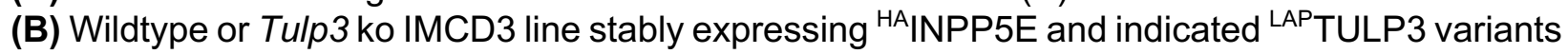
were grown to confluence and serum starved for 36 hours before fixation. Fixed cells were immunostained for endogenous Arl13b, HA (INPP5E) or endogenous Gpr161 along with GFP (TULP3) and acetylated tubulin (AcTub) and counterstained for DNA.

(C) Arl13b/ HA/Gpr161 and GFP positive cilia were counted from two experiments, and total counted cells are $>200$ for each condition. Data represent mean \pm SD.

(D) Violin plot shows mean ciliary intensities of Arl13b and HA from different cell types used in (B) (50 cilia each for Arl13b and 30 cilia each for ${ }^{\mathrm{HA}}$ Inpp5e).

(E) Cilia lengths were measured from different cell types used in ( $A$ ) by acetylated tubulin immunostaining (50 cilia each).

Scale, $5 \mu \mathrm{m}$. ${ }^{* * *}, \mathrm{p}<0.0001 ;{ }^{* *}, \mathrm{p}<0.01 ;{ }^{*}, \mathrm{p}<0.05$; ns, not significant. Arrows indicate cilia positive for the indicated proteins while arrowheads indicate negative cilia. 


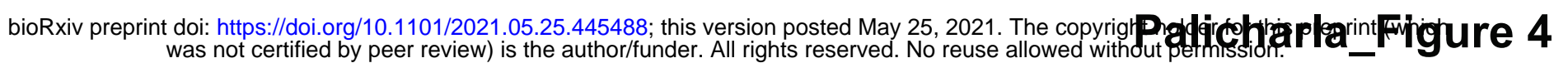

A C

TULP3 TULP3 $3{ }^{K R}$ TULP3 $3^{\text {mut12 }}$ TULP3 $3^{\text {KR/mut12 }}$
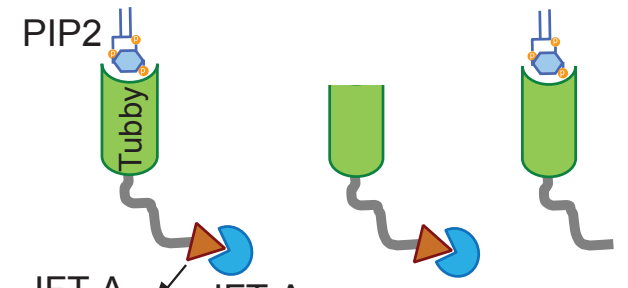

binding

motif

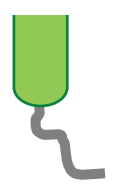

B

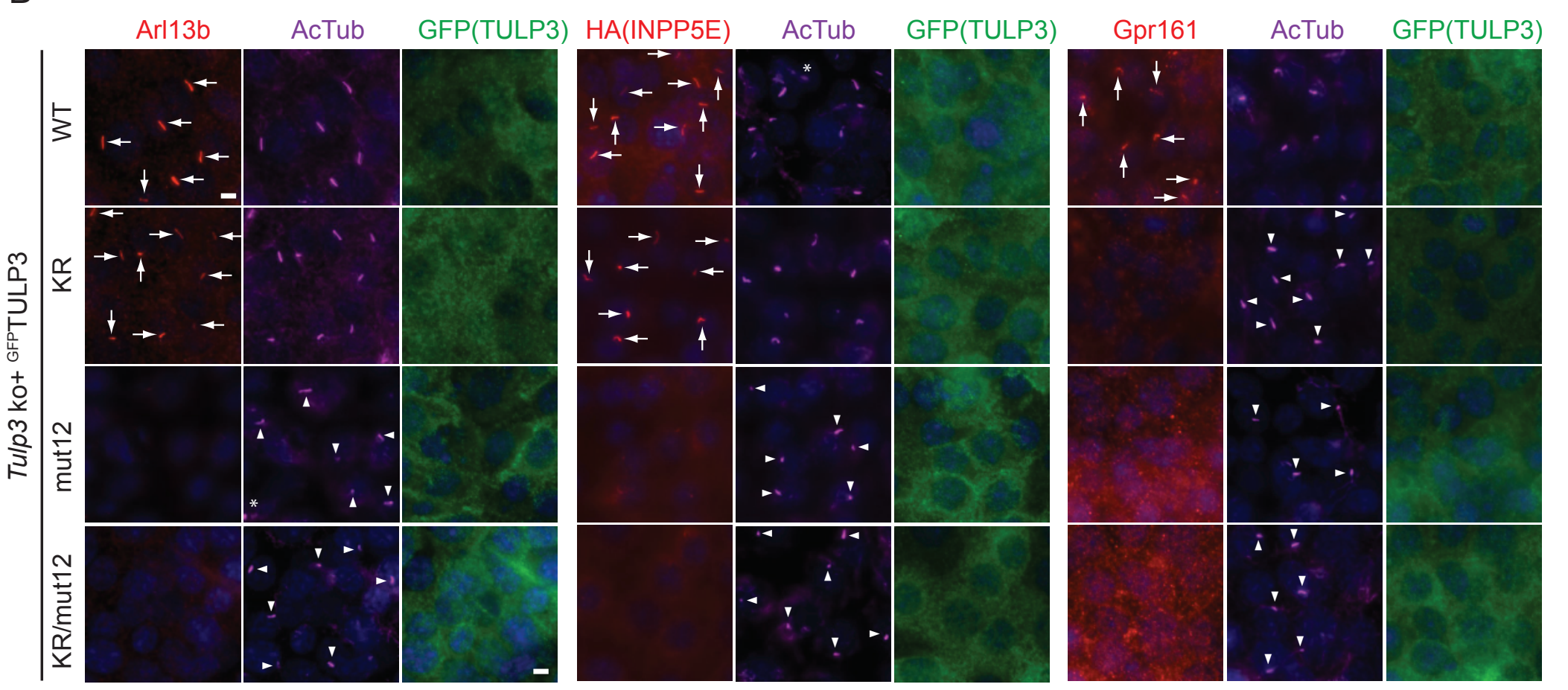

$\mathrm{D}$

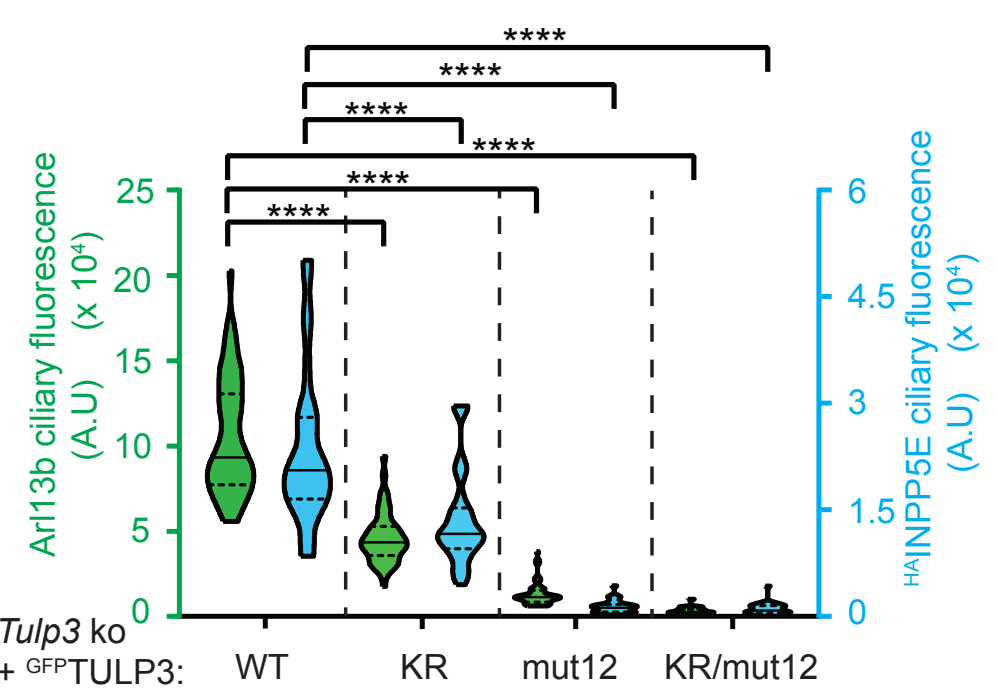

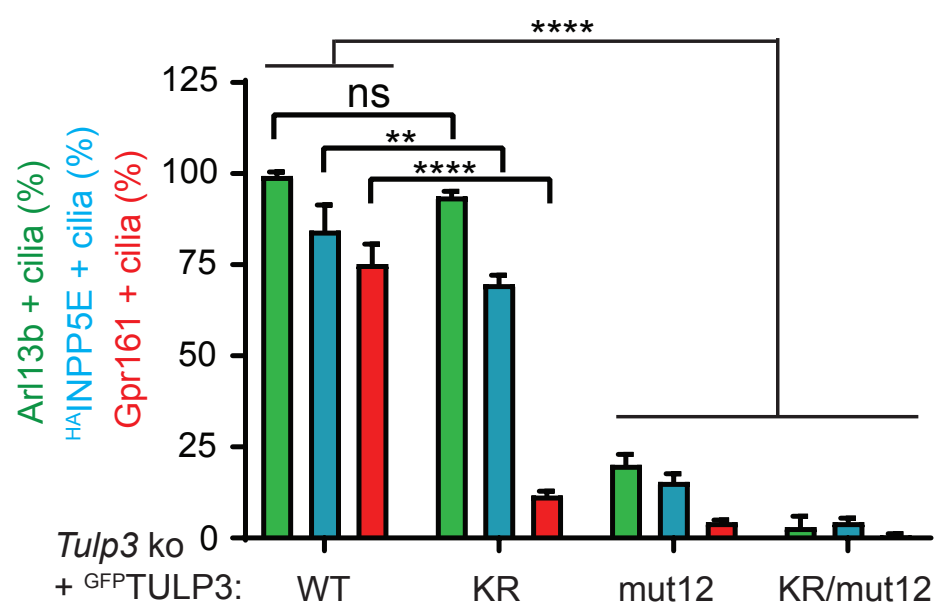

E

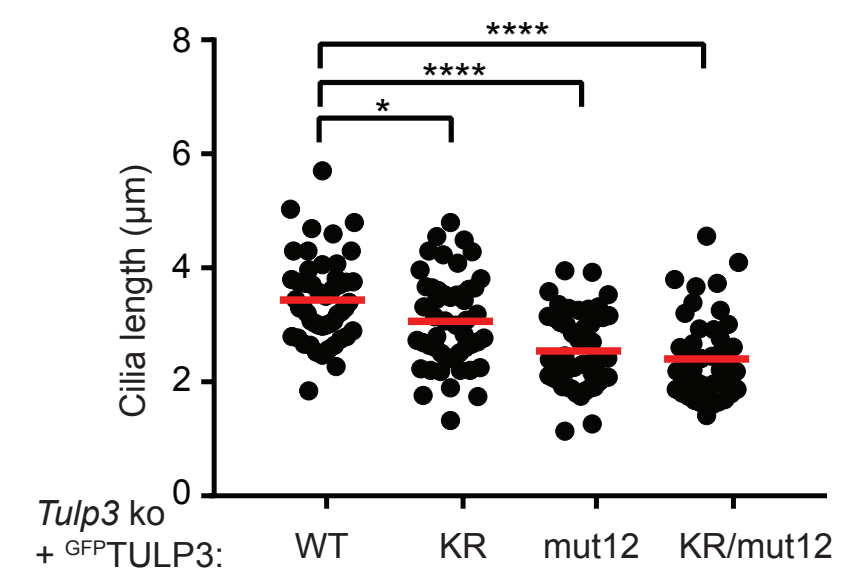


Figure 5: Tubby domain of TULP3 directly interacts with ARL13B.

(A) Flowchart showing steps involved in proximity biotinylation assay in cells.

(B) T-REx 293 cells were co-transfected with GFP-TEV-S-tagged TULP3 full-length (FL) or Nterminus (NT) or C-terminus (CT) along with BirA*-Flag tagged ARL13B and processed as shown in (A). CD8 inker is a negative control and CD8-Fibrocystin (Fibro) CLS is a positive control (Badgandi et al., 2017). Final S-tag bead eluates were processed for immunoblotting with IRdye-680 tagged streptavidin to detect biotinylation. S-tag antibody blot shows efficiency of pull down. Inputs were blotted with the indicated antibodies to detect expression. $\alpha$ Tubulin is used as a loading control. Mean \pm SD values indicate Biotin/S-tag ratios normalized to CD8 linker control.

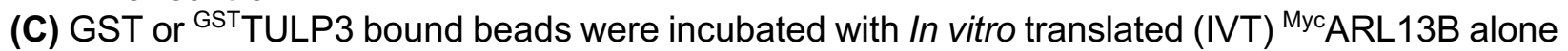
or in the presence of excess of MBP or MBPTULP3 or MBPTubby protein in Lap100N at room temperature for 1 hour. Beads were washed, eluted and immunoblotted with the indicated antibodies. MBP, maltose binding protein.

(D) In vitro translated GFPARL13B was incubated with GST, ${ }^{\text {GST }}$ TULP3 full length, ${ }^{\text {GST TULP3 NT }}$ or GSTTULP3 CT in LAP100N buffer at room temperature for one hour. Beads were processed for immunoblotting with the indicated antibodies.

(E) Bacterially purified GST or GSTTULP3 protein bound to glutathione sepharose beads were incubated with insect cell purified GFP tagged ARL13B in LAP150N buffer at $4^{\circ} \mathrm{C}$ for $1 \mathrm{~h}$. Beads were washed and immunoblotted with GFP antibody to show direct in vitro interaction. Ponceau staining was performed to show GST or GSTTULP3 on beads. Input shows flowthrough immunoblotted with GFP antibody to show the presence of GFPARL13B in the reaction.

(F) Same as in (E), but tubby domain of TULP3 $\left({ }^{\mathrm{GST}}\right.$ TULP3 $\left.{ }^{\mathrm{CT}}\right)$ and insect purified GFP tagged Nterminus of ARL13B (1-298) (See Figure 8 schematic) used for in vitro binding reactions.

Number of experiments performed denoted by " $n$ ". Asterix in immunoblots in (C) and (D) refer to nonspecific band in anti-MYC blot in IVT reactions. ${ }^{* * *}, p<0.0001 ;{ }^{* *}, p<0.01$; ${ }^{*}, p<0.05$; ns, not significant. 
A

Transfect

GFP-TEV-S tag-TULP3

+ BirA*-Flag-ARL13B

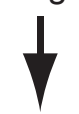

Biotin

$20 \mu \mathrm{M}$

(8-12 h)

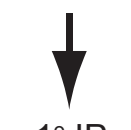

$1^{\circ} \mathrm{IP}$

anti-GFP

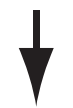

TEV

Cleavage

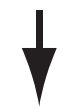

$2^{\circ}$ IP

anti-S tag
B

GFP-TEV-S tagTULP3:

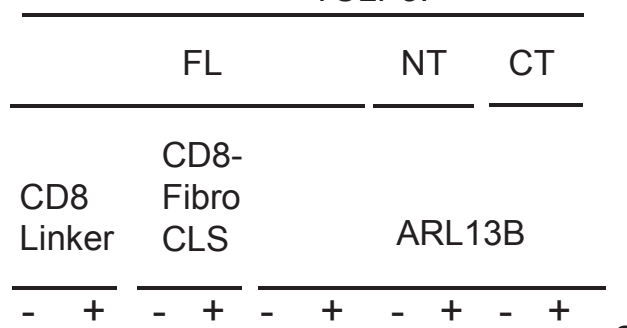

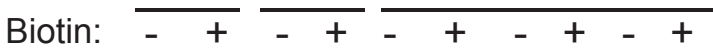
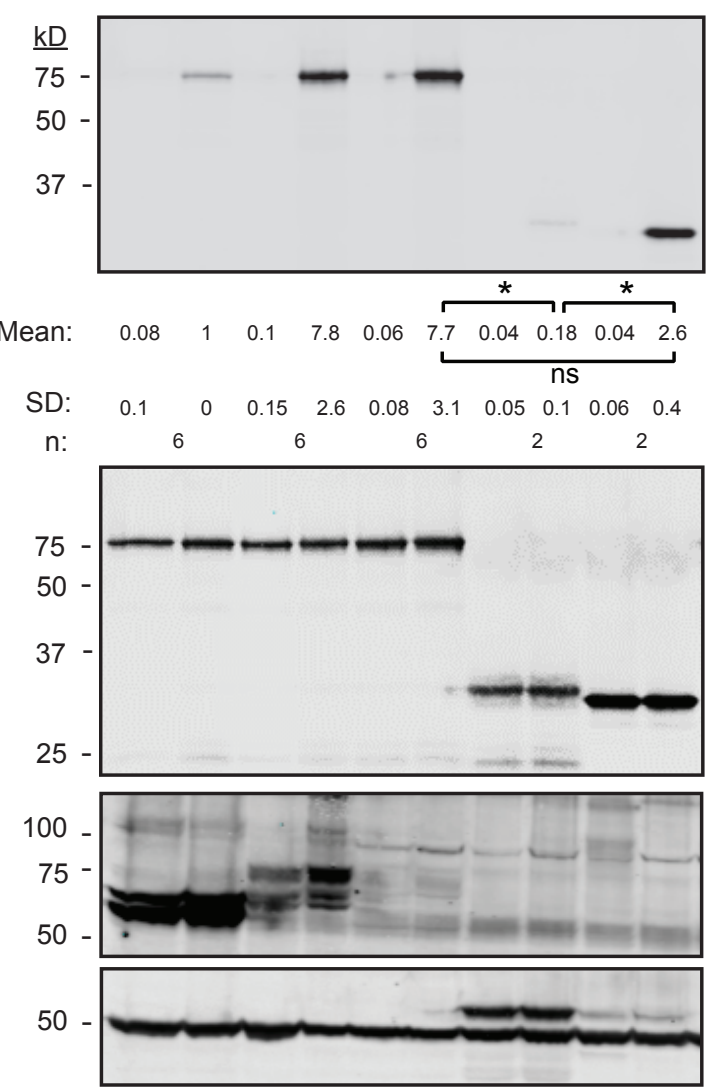

E
a-Tubulin

WB: S tag

INPUT:

WB: Flag

F
$\underline{\mathrm{S} \operatorname{tag} 2^{\circ}} \underline{\underline{\mathrm{IP}}}$

WB: Biotin

C

\begin{tabular}{cccccc} 
& \multicolumn{5}{c}{${ }^{M y c} A R L 13 B$} \\
\cline { 2 - 5 } MBPTUBBY & - & - & - & - & + \\
MBPTULP3 & - & - & - & + & - \\
MBP & - & - & + & - & - \\
GSTTULP3 & - & - & + & + & + \\
GST & + & - & - & - & -
\end{tabular}

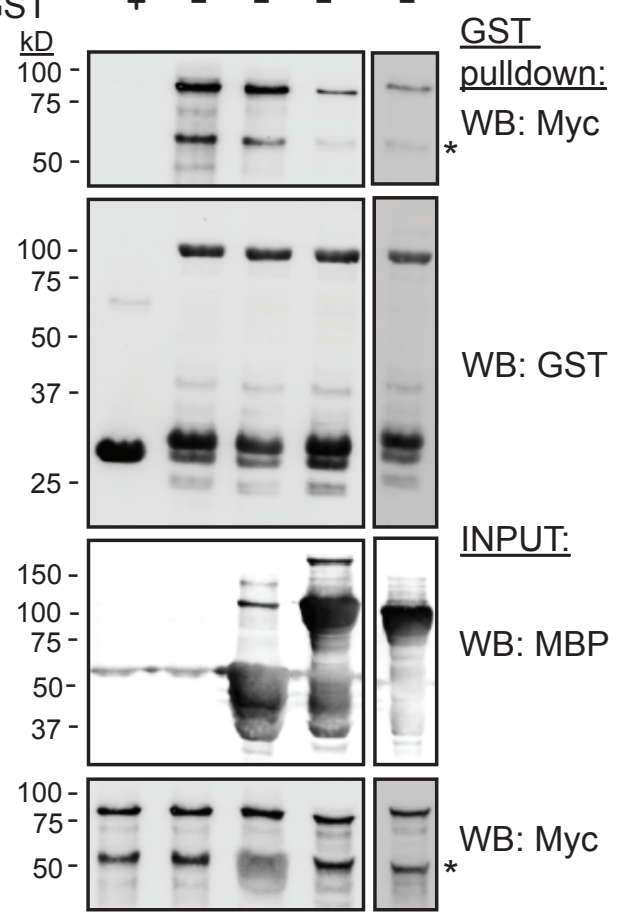

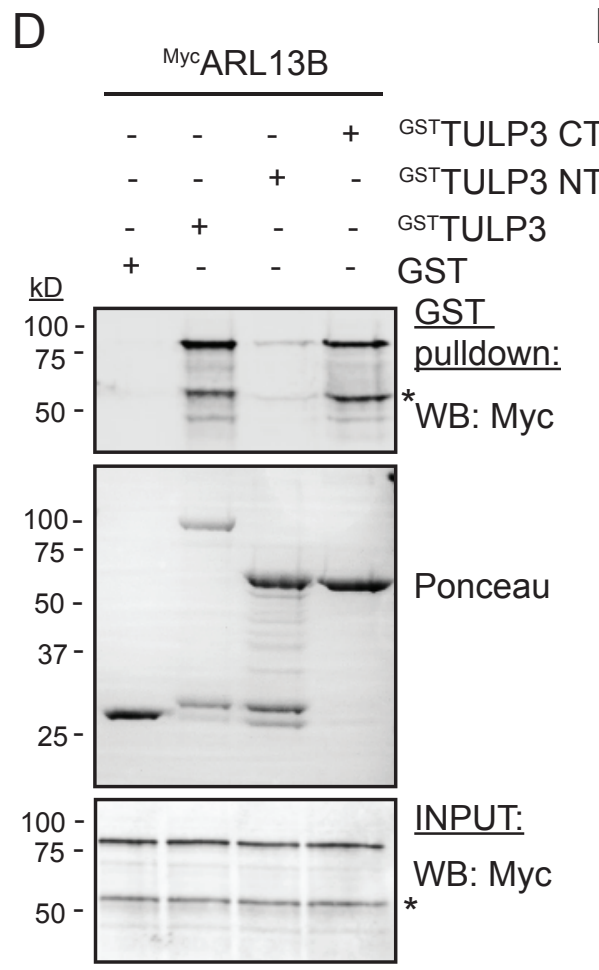

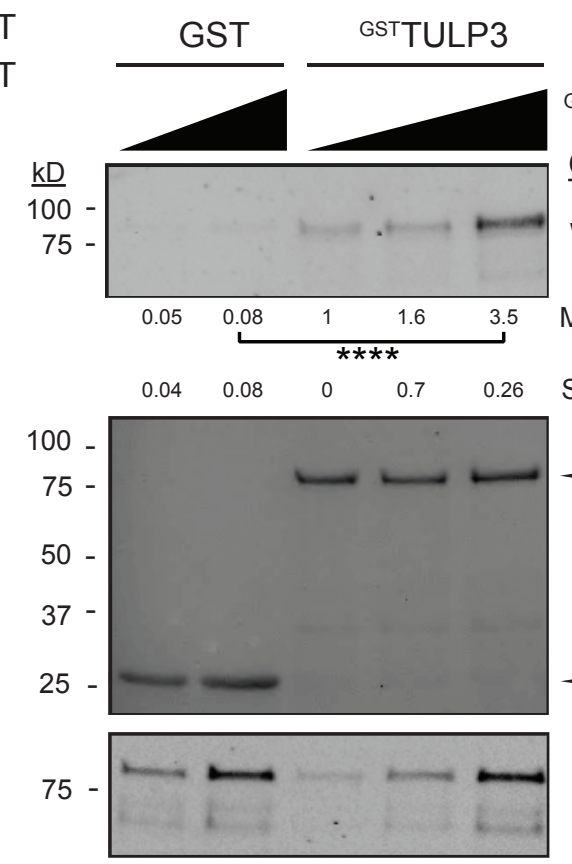

GFPARL13B
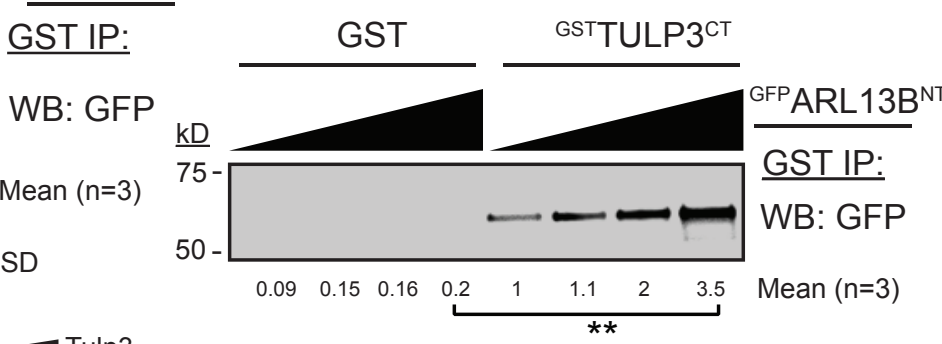

Tulp3

$\begin{array}{lllllllll}0.01 & 0.01 & 0.05 & 0.05 & 0 & 0.14 & 0.6 & 1.13 & \text { SD }\end{array}$

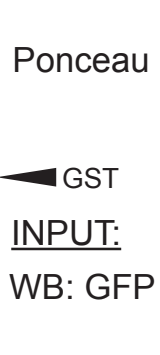

Tulp3

Ponceau

GST

INPUT:

WB: GFP 
Figure 6: Lysine K389 in TULP3 is required for interaction with its cargoes.

(A) T-Rex 293 cells were co-transfected with GFP-TEV-S-tagged TULP3 wild type (WT) or K389l mutant along with BriA*-Flag tagged ARL13B and processed as shown in 5A-B. " $n$ " indicates number of experiments performed. Data represent mean $\pm S D$.

(B) Increasing amounts of insect cell purified GFPARL13B (1-298) (NT) protein was incubated with either GST, GSTTULP3 or GSTTULP3 ${ }^{\mathrm{K} 389 \mathrm{l}}$ as in 5E-F. " $\mathrm{n}$ " indicates number of experiments performed. Data represent mean \pm SD.

(C) Tandem affinity purification of RPE cells stably expressing LAP tagged wild type TULP3 or K389I mutant was performed, and the silver stain shows the presence of multiple IFT-A components in the pull downs.

(D) Samples from (C) were subjected to mass spectrometry to identify the proteins in the pulldown complexes. Table shows the IFT-A components identified along with respective peptide counts and percentage of coverage.

(E) Samples from (C) were subjected to immunoblotting with anti-IFT139 (THM1) and S-tag antibody.

(F) Equal amounts of GST, ${ }^{\text {GSTTTULP3 }}$ or ${ }^{\mathrm{GST}}{ }^{\mathrm{T}}$ TULP3 ${ }^{\mathrm{K} 3891}$ protein was added to lipid strips to test for lipid binding properties of the proteins.

(G) IMCD3 cells stably expressing ${ }^{\text {LAP }}$ TULP $3^{\text {WT }}$ or ${ }^{\text {LAPTULP }}{ }^{\mathrm{K} 3891}$ were serum starved for 36 hours before fixing and immunostained for GFP, Acetylated tubulin and DNA. Insets hsow TULP3 localization in cilia.

Scale, $5 \mu \mathrm{m}$. Inset scale, $1 \mu \mathrm{m} .{ }^{* *}, \mathrm{p}<0.01$. See also Figure S4. 
A

GFP-TEV-S tagTULP3:

$\begin{array}{lll}\text { WT } & \text { K389I } \\ \text { CD8 CD8- } & +\end{array}$

BirA*-Flag: Linker FibroCLS ARL13B ARL13B

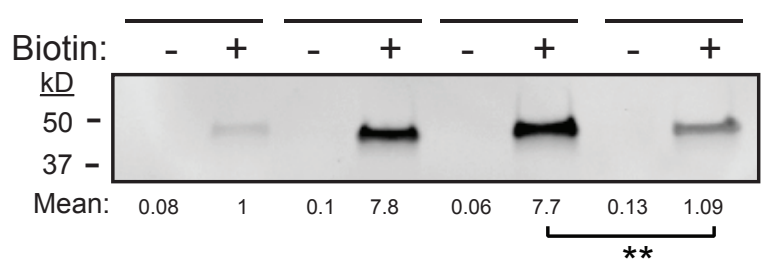

S tag 2이:

WB: Biotin

$\begin{array}{lllllllll}\text { SD: } & 0.1 & 0 & 0.15 & 2.6 & 0.08 & 3.1 & 0.21 & 0.75\end{array}$
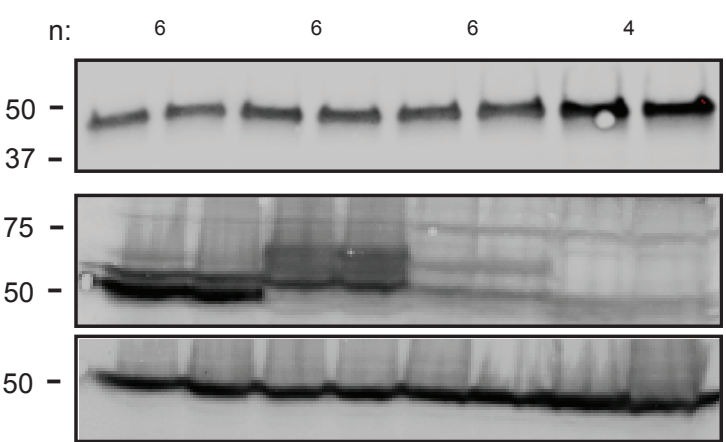

WB: S tag

INPUT:

WB: Flag

D

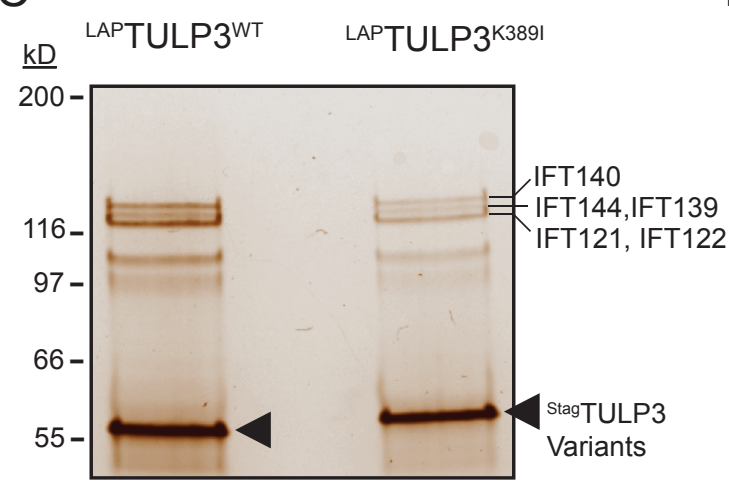

a-Tubulin
B

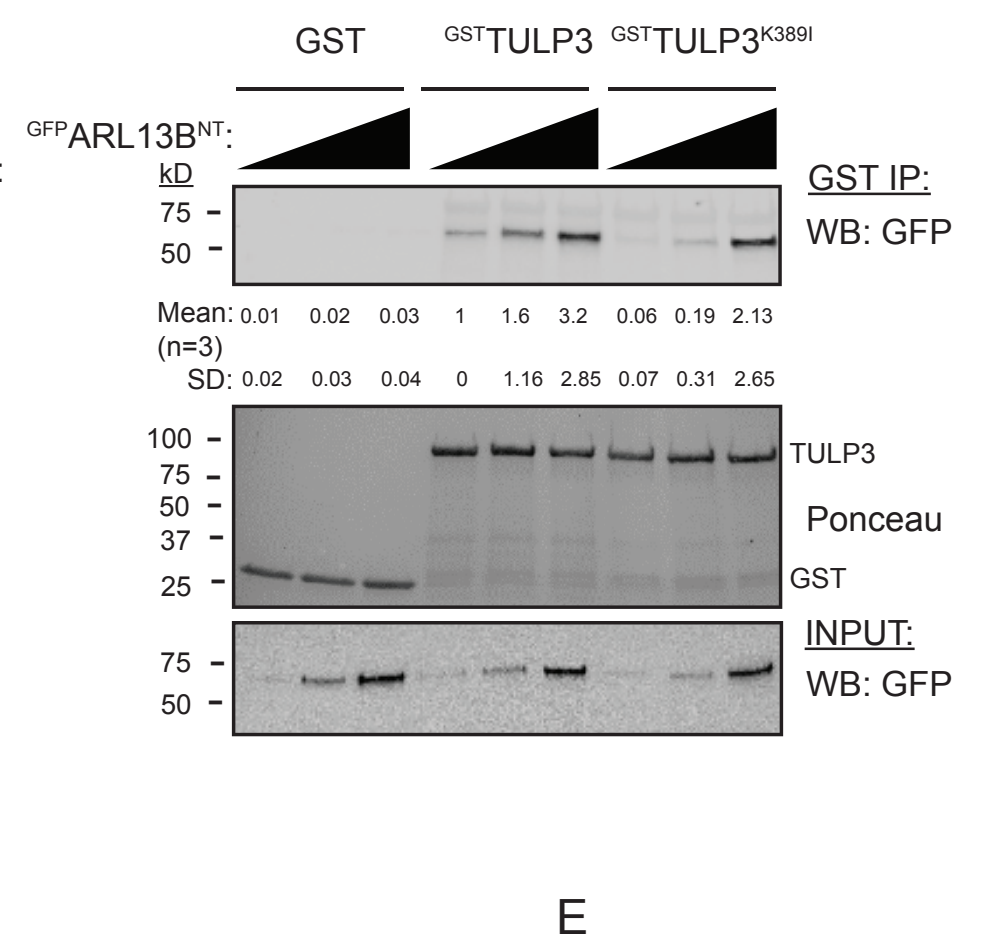

\begin{tabular}{lllll}
\hline Protein & $\begin{array}{c}\text { NCBI } \\
\text { Gene ID }\end{array}$ & MW (KDa) & $\begin{array}{c}\text { Spectral } \\
\text { count } \\
\text { (Unique } \\
\text { peptides) }\end{array}$ & $\begin{array}{c}\% \\
\text { Coverage }\end{array}$ \\
\hline TULP3 & 7289 & 49 & $46(18)$ & 31 \\
IFT121 & 57539 & 134 & $16(10)$ & 9 \\
IFT139 & 73668 & 150 & $13(9)$ & 7 \\
IFT144 & 213081 & 151 & $8(8)$ & 6 \\
IFT122 & 81896 & 134 & $8(4)$ & 4 \\
IFT140 & 106633 & 82 & $4(4)$ & 5 \\
\hline
\end{tabular}

$E$

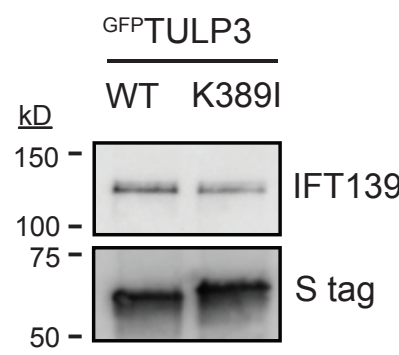

$\mathrm{F}$ GST GSTTULP3 ${ }^{W T}$ GSTTULP3 ${ }^{\mathrm{K} 3891}$

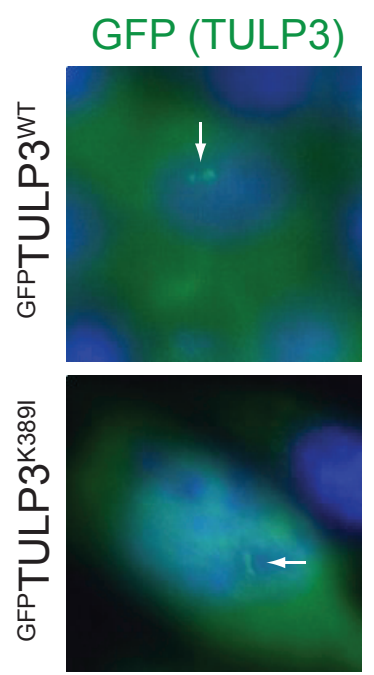

AcTub

Merge/DNA
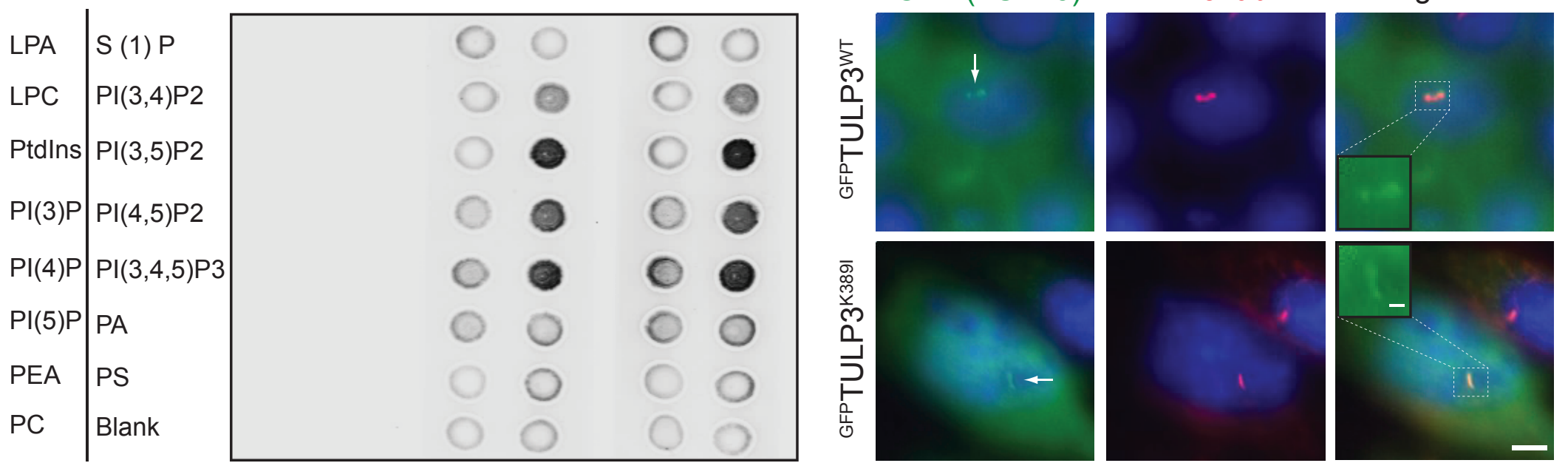


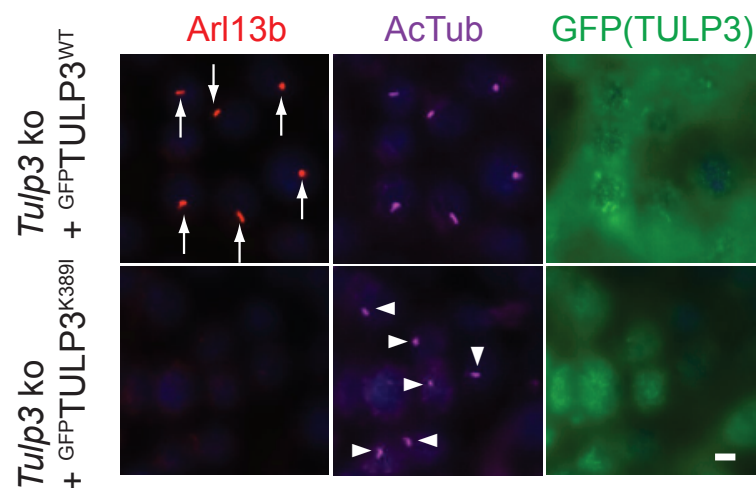

B

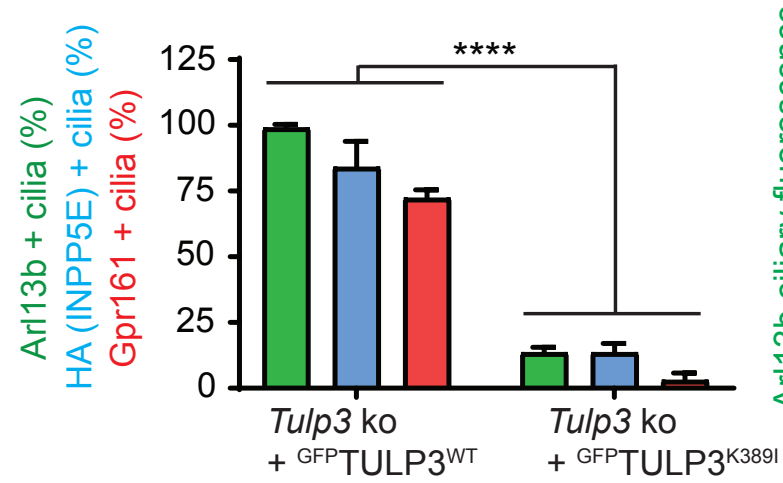

C
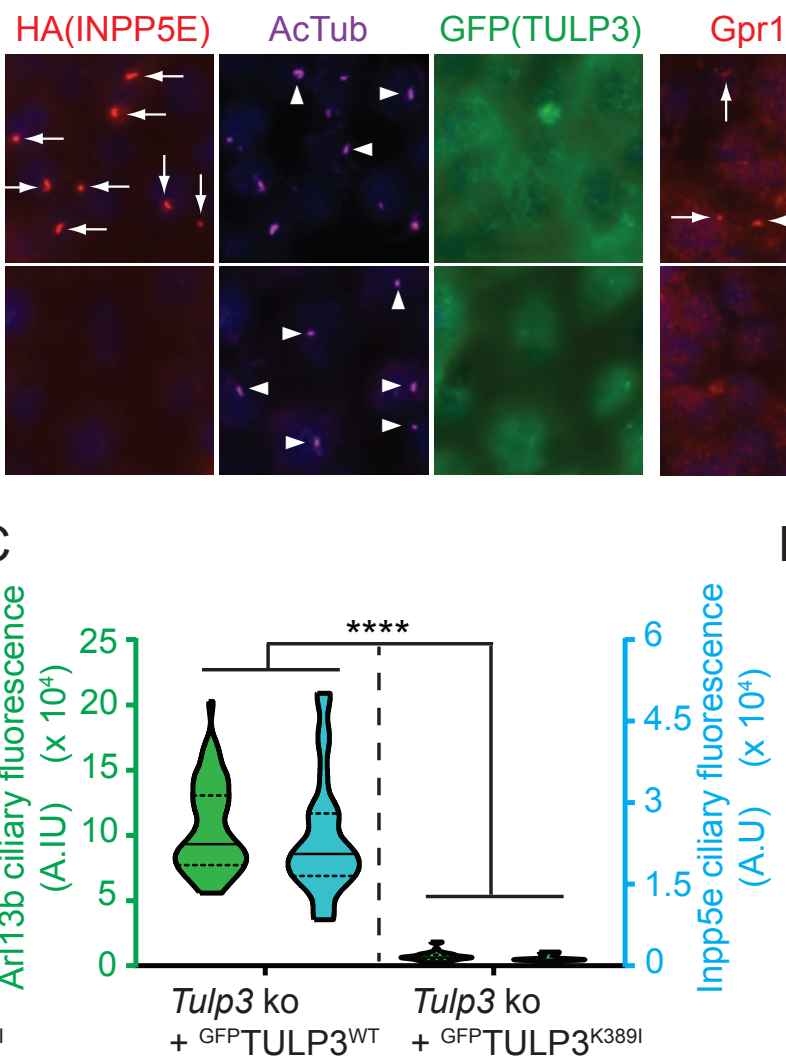
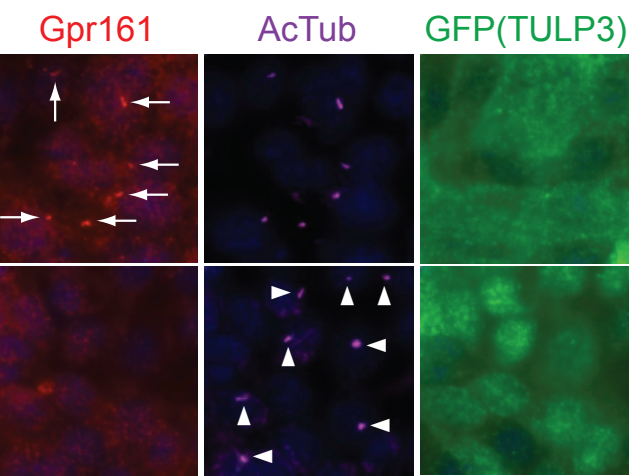

D

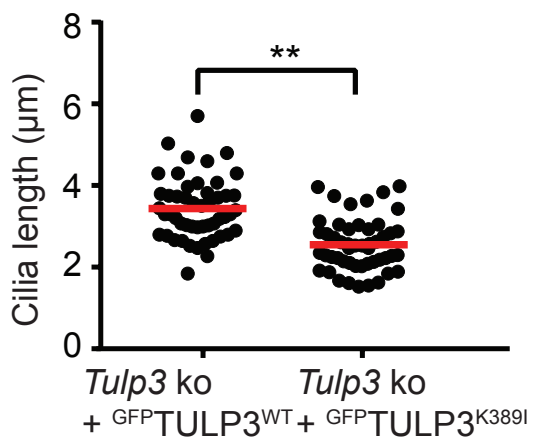

$\mathrm{E}$

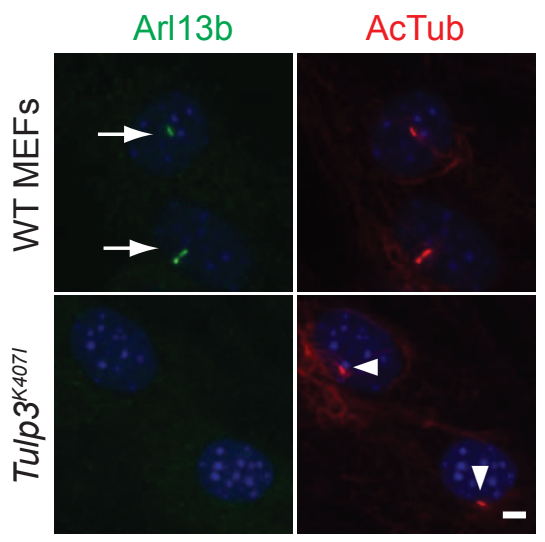

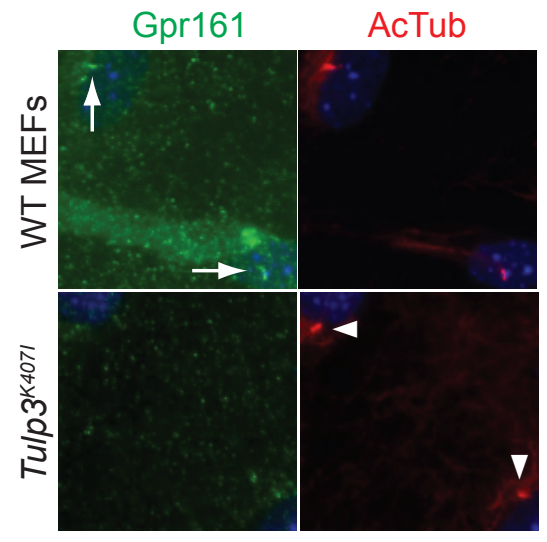

$\mathrm{F}$

Figure 7: Lysine 389 in TULP3 is required for ciliary cargo trafficking.

(A) Wildtype or Tulp3 ko IMCD3 line stably expressing HA INPP5E and LAP-tagged wild type or K389I mutant of TULP3 were grown till confluence. Cells were serum starved for $36 \mathrm{~h}$ before fixation and then immunostained for Arl13b or HA or Gpr161 along with GFP, acetylated tubulin (AcTub) and counterstained for DNA.

(B) Arl13b/HA/Gpr161 in GFP(TULP3) positive cilia were counted from two experiments, and total counted cells are $>200$ for each condition. Data represent mean \pm SD.

(C) Violin plot shows mean ciliary intensities of Arl13b and HA (50 cilia each for Arl13b and 30 cilia each for ${ }^{\mathrm{HA}}$ INPP5E).

(D) Cilia lengths were measured from by immunostaining for acetylated tubulin ( $>50$ cilia per condition).

(E) MEFs from wild type or Tulp $3^{K 4071}$ mice were serum starved for $24 \mathrm{~h}$ before fixation and immunostained for either Arl13b or Gpr161 along with acetylated tubulin (AcTub) and counterstained for DNA.

(F) Arl13b or Gpr161 positive cilia were counted from two experiments, and total counted cells are $>200$ for each condition. Data represent mean \pm SD.

Scale, $5 \mu \mathrm{m} .{ }^{* * * *}, \mathrm{p}<0.0001 ;{ }^{* *}, \mathrm{p}<0.01$; ns, not significant. Arrows indicate cilia positive for the indicated proteins while arrowheads indicate negative cilia. 
Figure 8: Arl13b domains required for Tulp3 interaction and ciliary localization.

(A) Schematic representation of different ARL13B truncations tested for in vitro TULP3 binding ("TULP3 binding") or stably expressed in immortalized Arl13b $b^{h n}$ MEFs along with HA-tagged INPP5E to test for ciliary trafficking ("cilia localization") and rescue of ARL13B function ("INPP5E rescue"), respectively. $\sim 30-100$ cilia counted for cilia localization and HA-tagged INPP5E positive cilia. Arl13b $b^{\text {hn }}$ MEFs had no HA-tagged INPP5E in cilia. \#, data from

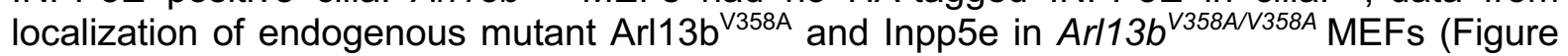
S5B) (Gigante et al., 2020).

(B) Bacterially purified GST or GSTTULP3 protein bound to glutathione Sepharose beads was incubated with in vitro translated (IVT) Myc tagged truncations of ARL13B in Lap150N buffer at room temperature for $1 \mathrm{~h}$. Beads were washed, eluted and immunoblotted with Myc

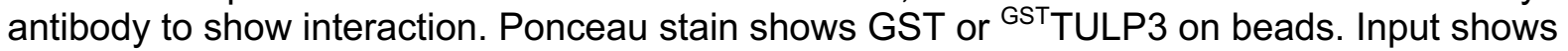
flowthrough immunoblotted with GFP antibody to show the presence of GFPARL13B in the reaction. Conserved palmitoylation motif residues with respect to the palmitoylated Cysteine are shown in each deletion (Ren et al., 2008; Weng et al., 2017)

(C) Alignment of N-terminus region of ARL13B preceding the GTPase domain and mutants tested for TULP3 binding and for ciliary localization. ARL13B protein IDs: Hs, homo sapiens NP_001167621.1; Mm, Mus musculus NP_080853.3; Rn, Rattus norvegicus NP_001100571.1; Dr, Danio rerio NP_775379.1; Cr̆, Chlamydomonas reinhardtii A8INQ0.1. Amphipathic helix for the human protein drawn using HeliQuest (Gautier et al., 2008). Arrow indicates hydrophobic moment.

(D) Bacterially purified GST or GSTTULP3 protein bound to glutathione Sepharose beads was tested for binding with in vitro translated Myc tagged full length ARL13B and indicated mutants as in (B).

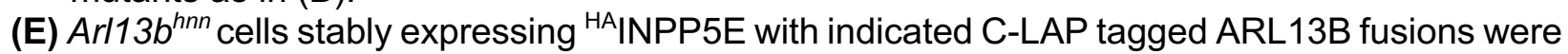
serum starved for $48 \mathrm{~h}$ before fixing and immunostained for GFP, HA (INPP5E), acetylated tubulin (AcTub) and counterstained for DNA. Quantification in (A) and Figure S5A.

Scale, $5 \mu \mathrm{m}$. ${ }^{* * * *}, p<0.0001 ;{ }^{* *}, p<0.001 ;{ }^{*}, p<0.05$; ns, not significant. Arrows indicate cilia positive for the indicated proteins while arrowheads indicate negative cilia. See also Figure S5. 


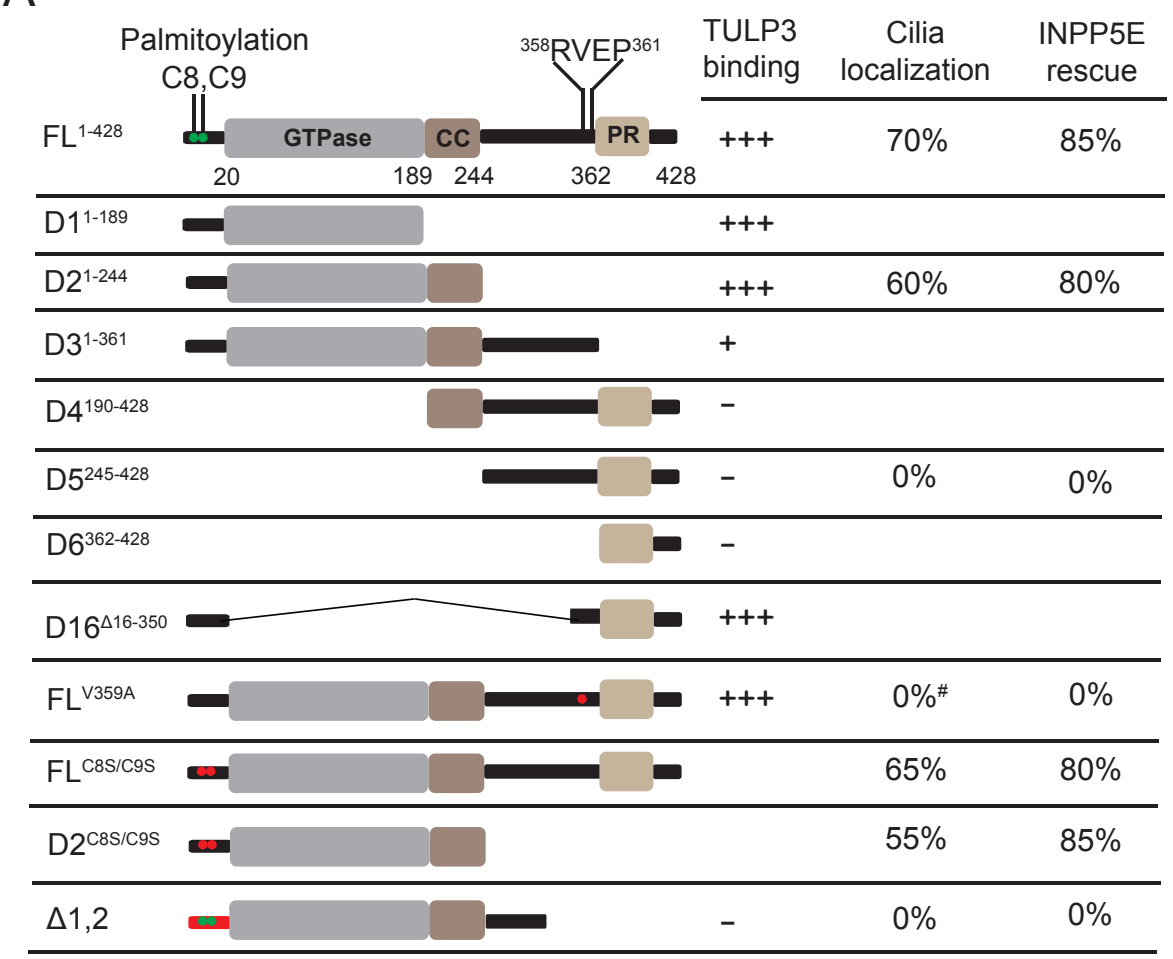
B

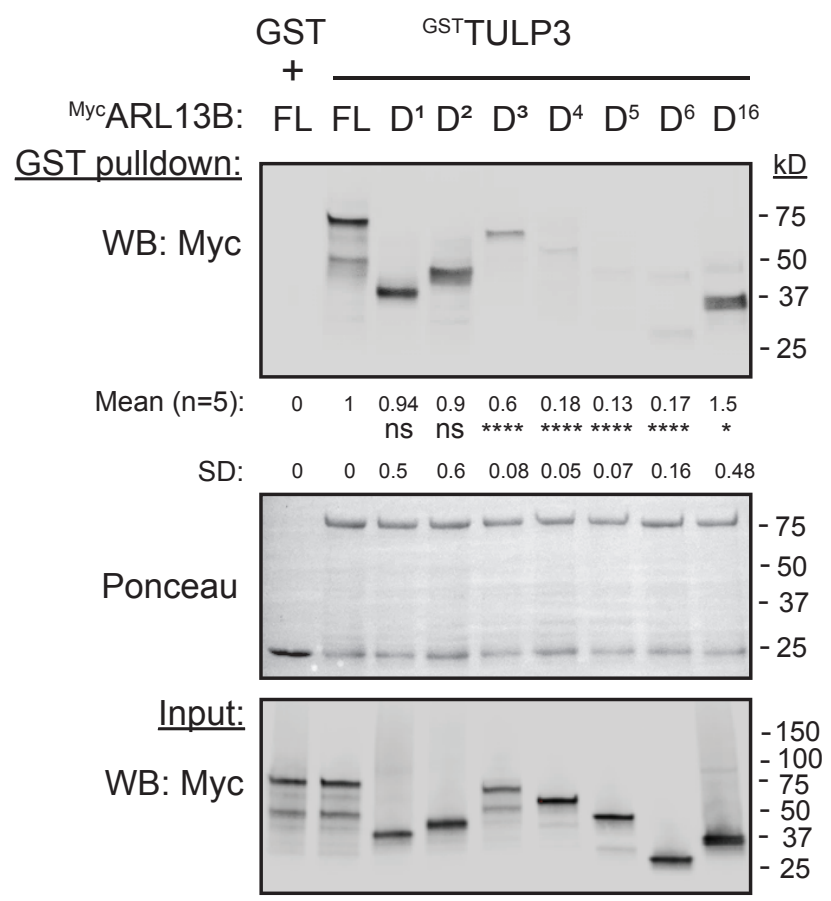

C
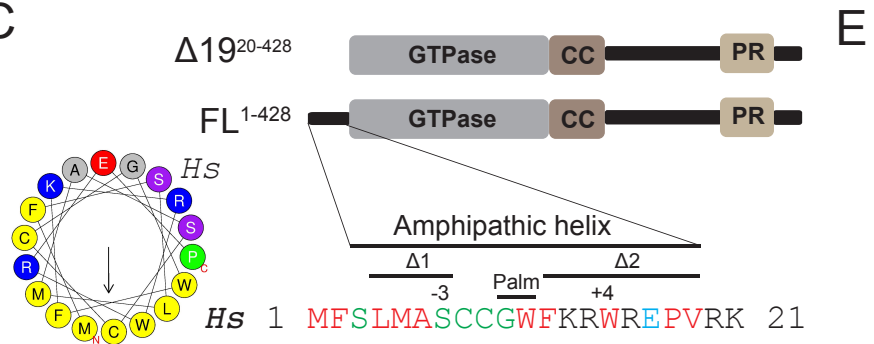

Mm 1 MFSLMANCCNLFKRWREPVRK 21

$\begin{array}{llll}R r & 1 & \text { MFNLMANCCNLFKRWREPVRK } 21\end{array}$

Dr 1 MFNLMANCCNWLKRWREPARK 21

Cr 1 MFGLLVNFYRFCR--KKTERK 19

$\star \star . \star$ :. : : : :

$\Delta 1,2: \stackrel{-3}{\mathrm{M}----\mathrm{A} S \overline{\mathrm{Calm}}}+\stackrel{+4}{\mathrm{CC}}-------\mathrm{PVRK}$

$\Delta 1: \stackrel{-3}{M}----$ A S $\overline{\text { Calm }} \stackrel{+4}{+4}$ KWFREPVRK

$\Delta 2: \stackrel{-3}{\stackrel{\text { Palm }}{M} \stackrel{+4}{\mathrm{MFSLMASC}}-------- \text { PVRK }}$

D

GST GSTTULP3

$\begin{array}{llllll}\text { MycARL13B: } & \text { FL FL } & \Delta 19 & \Delta 1,2 & \Delta 1 & \Delta 2\end{array}$

$\begin{array}{r}\text { GST pulldown: } \\ \text { WB: Myc }\end{array}-\frac{\mathrm{kD}}{-75}$

$\begin{array}{ccccccc}\text { Mean: } & 0.03 & 1 & 0.3 & 0.3 & 0.6 & 1.03 \\ & & & * * * * & * * * & * & \text { ns } \\ & & 4 & & 3 & 3 & 2\end{array}$

$\begin{array}{lllllll}\text { SD: } & 0.04 & 0 & 0.18 & 0.19 & 0.34 & 0.38\end{array}$

Ponceau

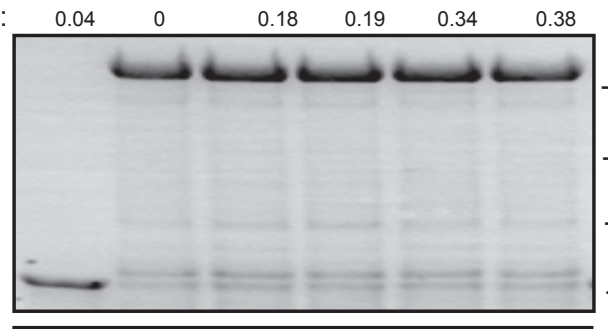

$\underline{\text { Input: }}$

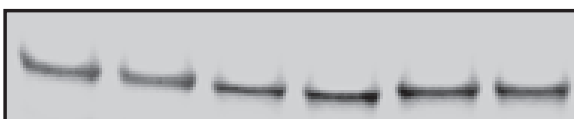

$E$

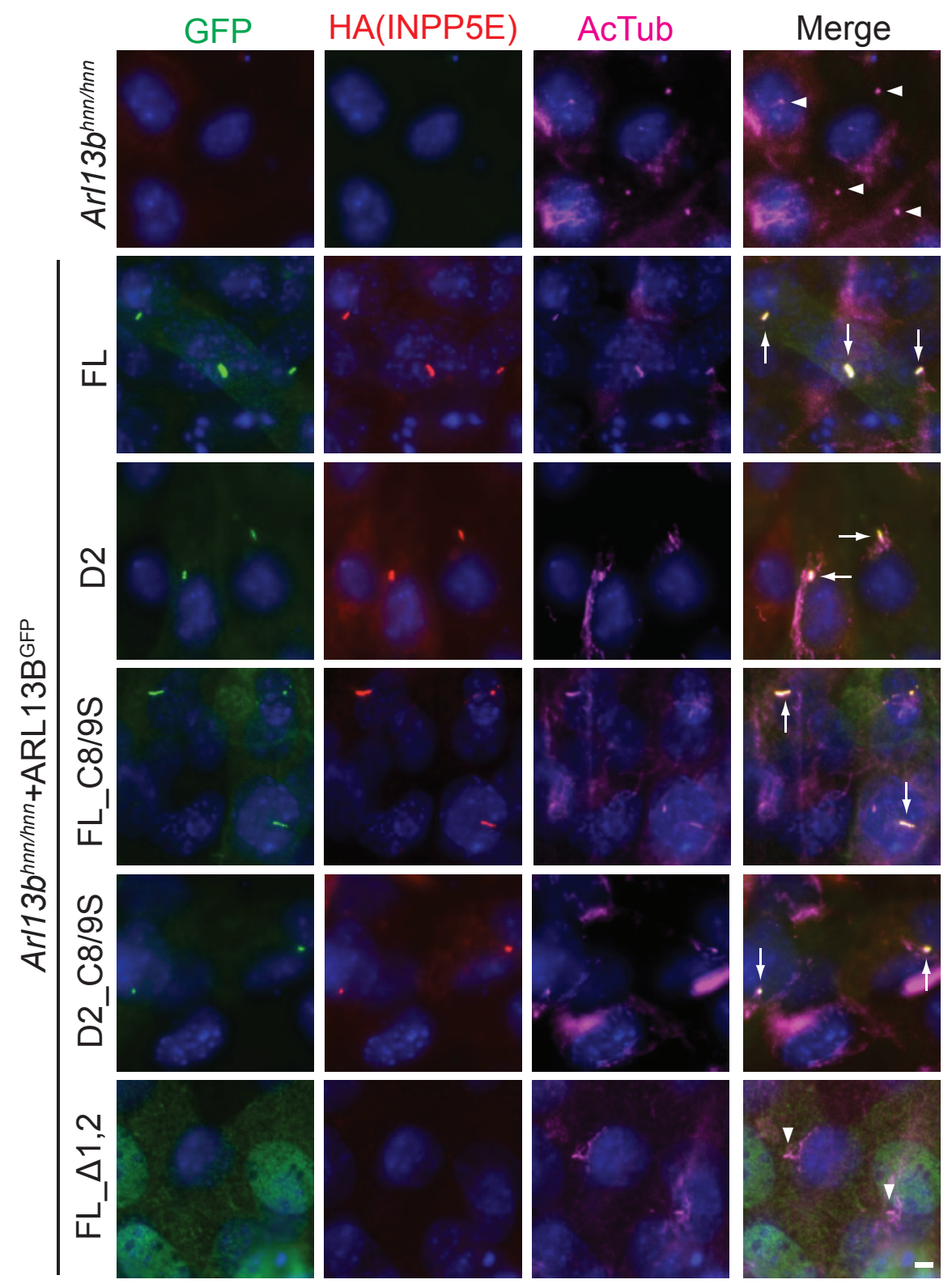


Figure S1 (Related to Figure 1). Tulp3 determines ciliary trafficking of Arl13b.

(A) MEFs from wild type or Tulp3 knockout (ko) mice were serum starved upon confluence for 24 $\mathrm{h}$ before fixation. Fixed cells were immunostained for Gpr161 (green) along with acetylated tubulin (AcTub, red) and counterstained for DNA (blue). Quantification shown in Fig. $1 \mathrm{~B}$.

(B) Genomic DNA was isolated from Tulp3 ko 3T3-L1, NIH 3T3 or IMCD3 and sequenced using exon3 specific primers. Open arrowheads point to mutations. $\mathrm{N}$, non-specific nucleotide.

(C-D) Wildtype and Tulp3 ko NIH 3T3 cells were grown to confluency and starved further for 48 $\mathrm{h}$ to promote ciliation before fixing. The fixed cells were immunostained for Arl13b (green) along with acetylated tubulin (red) and counterstained for DNA. Total counted cells are $>200$ for each condition. Data represent mean \pm SD.

(E-F) Wild type or Tulp3 knock out 3T3-L1 cells were grown to confluency and further cultured for 72 hours to promote ciliation. The cells were treated with $500 \mathrm{nM}$ Smo agonist (SAG) for 24 hours to induce Smo ciliary localization before fixing. Immunostaining was performed for Smo, Acetylated tubulin and DNA. Arrows indicate cilia positive for Smo while arrow heads indicate Smo negative cilia. Smo positive cilia from B were counted from two experiments $(E)$, and $>100$ cilia were counted for each condition. Data represent mean \pm SD.

Scale, $5 \mu \mathrm{m}$. ${ }^{* * *}, \mathrm{p}<0.0001$; ns, not significant. Arrows indicate cilia positive for the indicated proteins (Gpr161 (A) or Arl13b (C) or Smo (E)), while arrow heads indicate negative cilia. 
A

bioRxiv preprint doi: https://doi.org/10.1101/2021.05.25.445488; this version posted May 25, 2021. The copyrightholder for this preprint (mhich was not certified by peer review) is the author/funder. All rights reserved. No reuse allowed witholpathisipharla

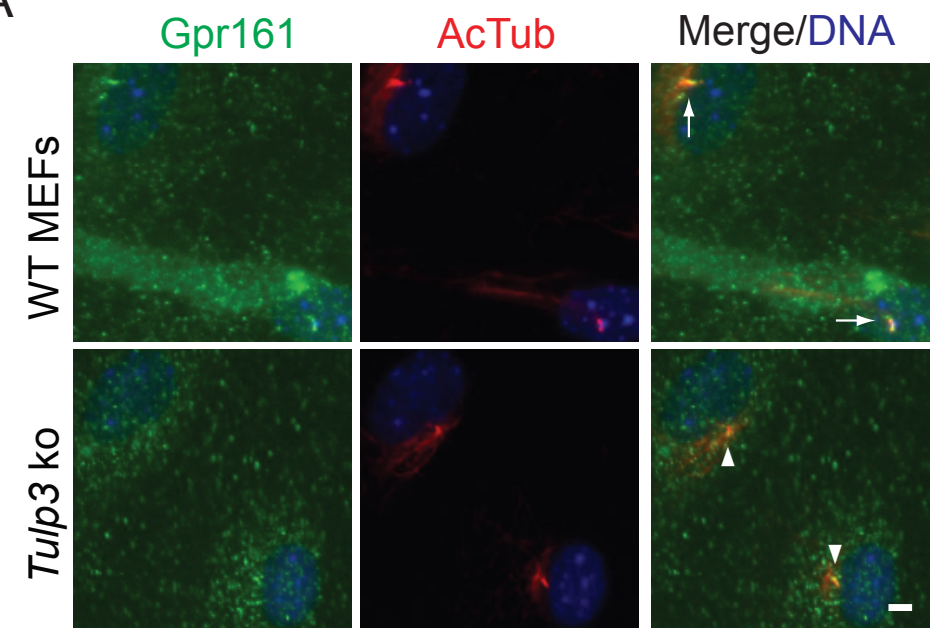

B

$\nabla \quad \nabla \nabla \nabla \nabla \nabla \nabla \nabla \nabla \quad \nabla \nabla$

$\nabla \nabla \nabla$

$\begin{array}{lllll}\nabla & \nabla & \nabla & \nabla\end{array}$ GT G G ACCNTN

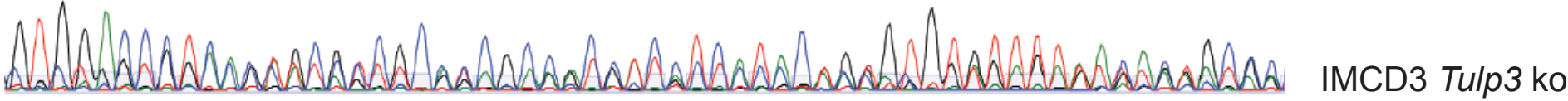

$\begin{array}{lll}\nabla & \nabla \nabla \nabla \nabla \nabla \nabla \nabla \nabla \nabla \nabla \nabla \nabla \nabla \nabla \nabla \nabla\end{array} \nabla \nabla \nabla \quad \nabla \nabla \nabla \nabla \nabla \nabla \nabla \nabla \nabla \nabla \nabla \nabla \quad \nabla \nabla \nabla \nabla \nabla \nabla \nabla \nabla \nabla \nabla \nabla \nabla \nabla$

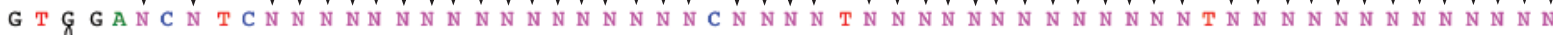

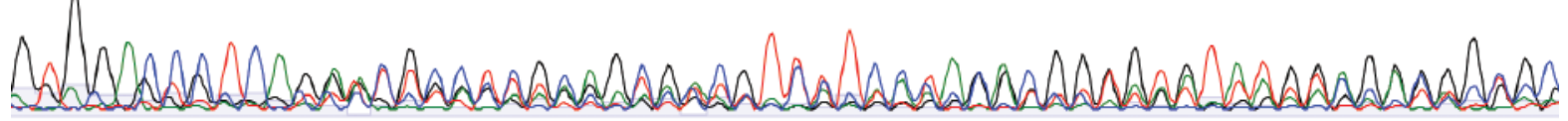

3T3-L1 Tulp3 ko G T G GAC C C TCANATGCCTCGNAGCGNCG TNNTCCTACACG TG TGNATTTAGACNNNNN

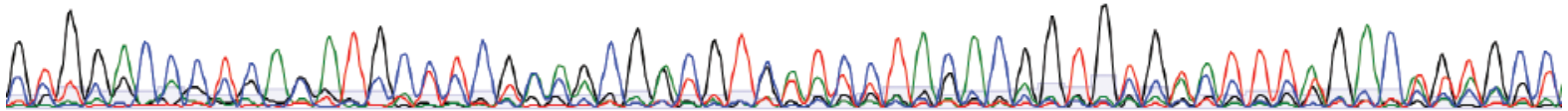
GTGGACCCTCAGATGCCTCGCAGCGACGTCATCCTACACGGTGTGTATTTAGACAGTGCC

NIH 3 T3 Tulp3 ko PAM

${ }^{\Delta}$ End of Exon 3

C
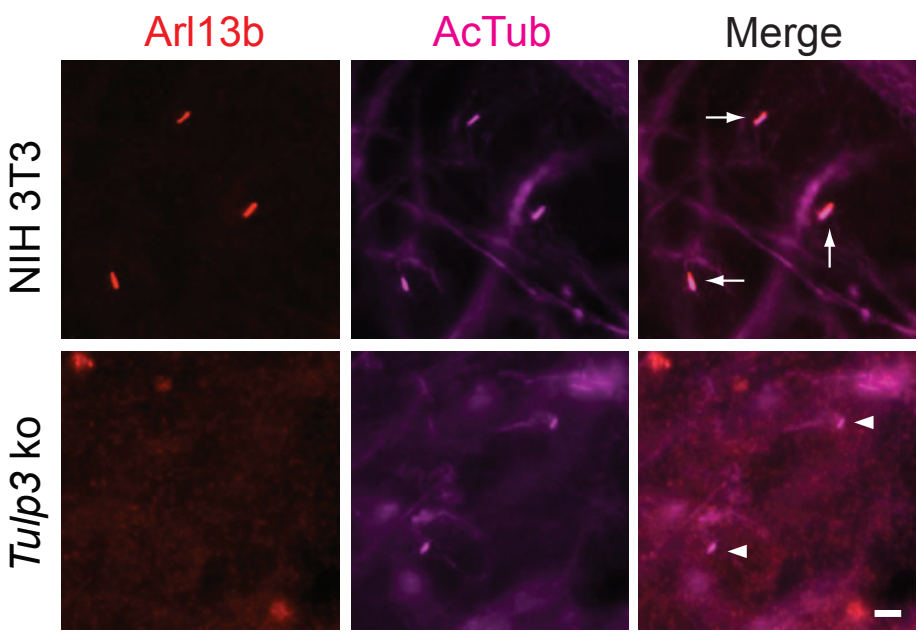

$D$

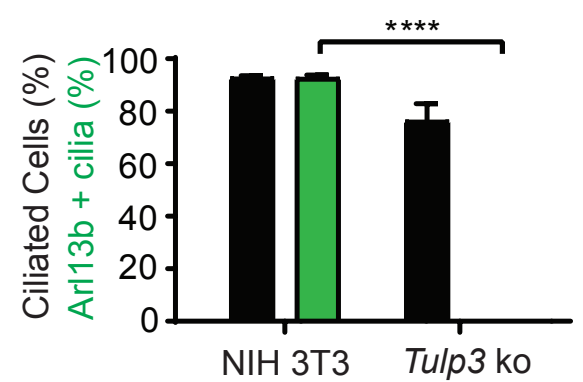

$\mathrm{F}$
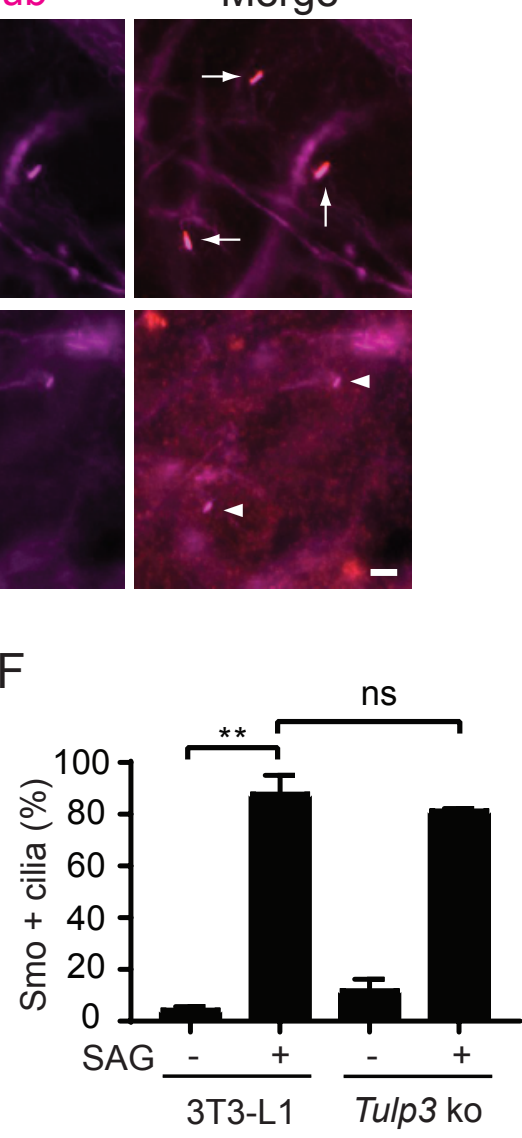

$E$
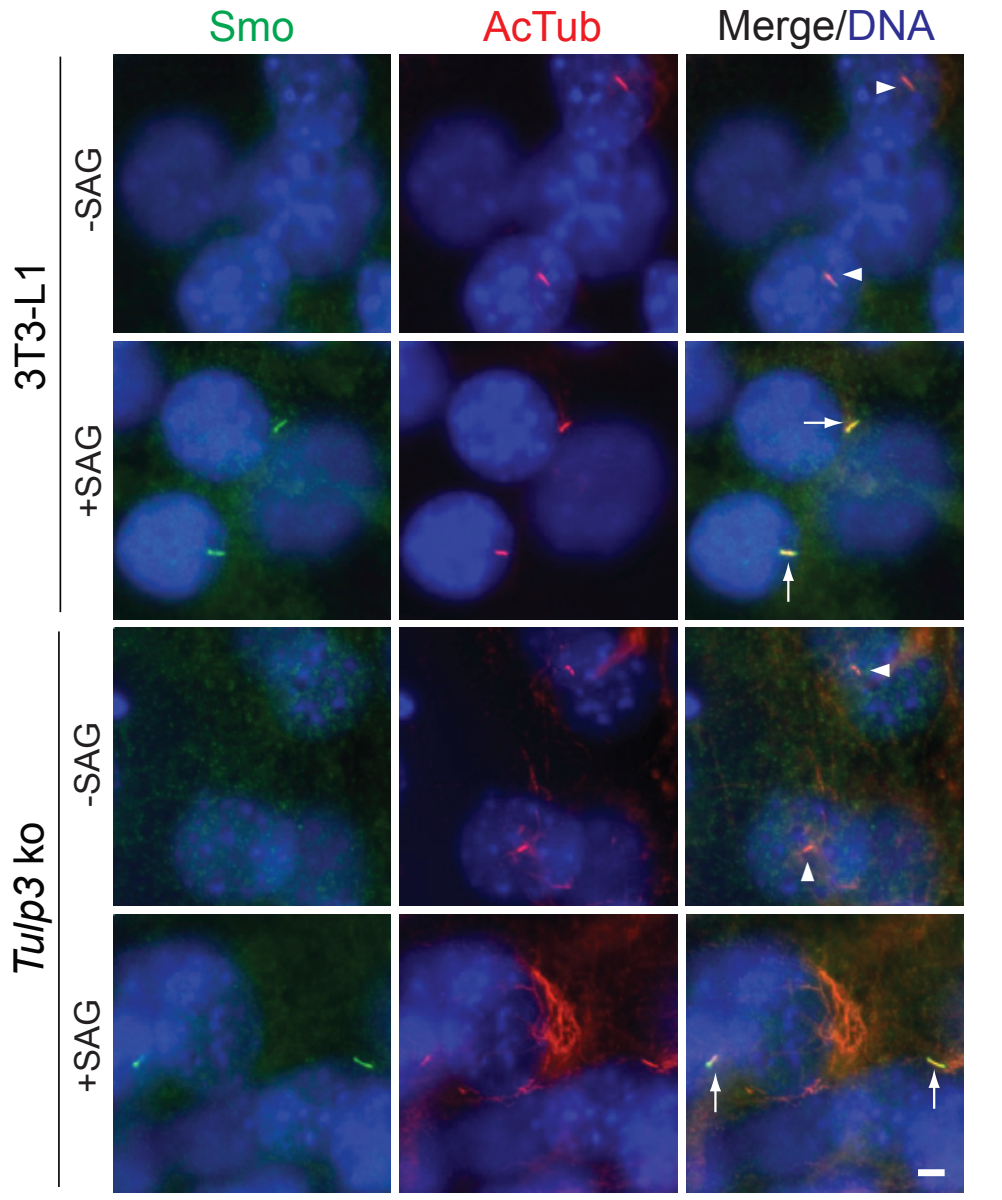


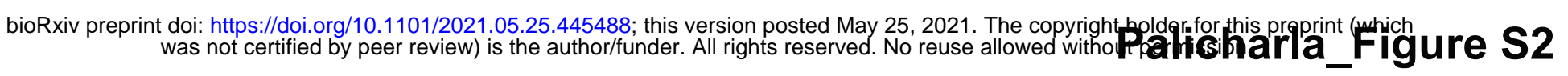

A

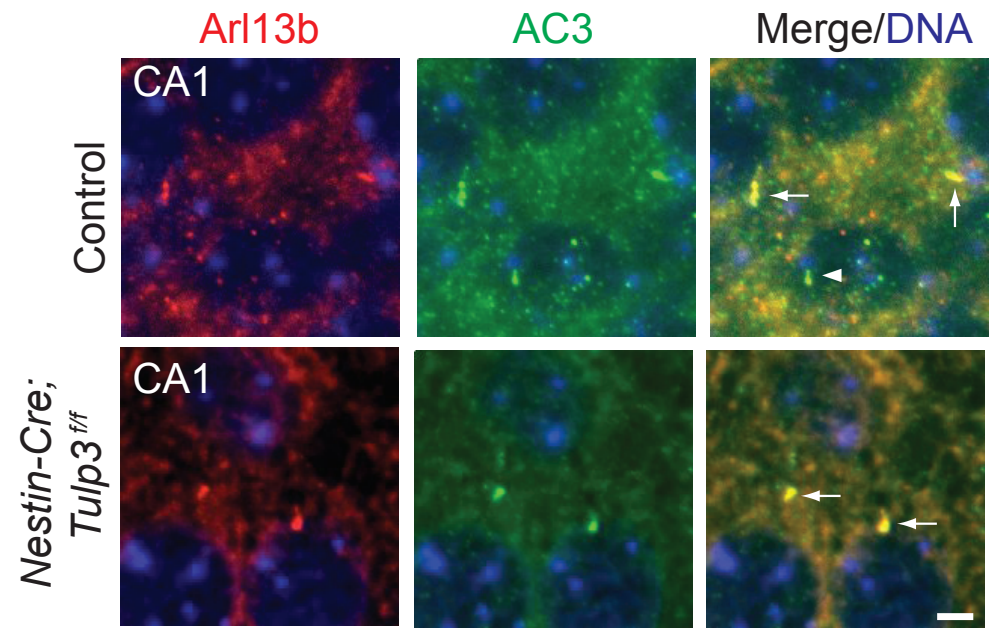

B

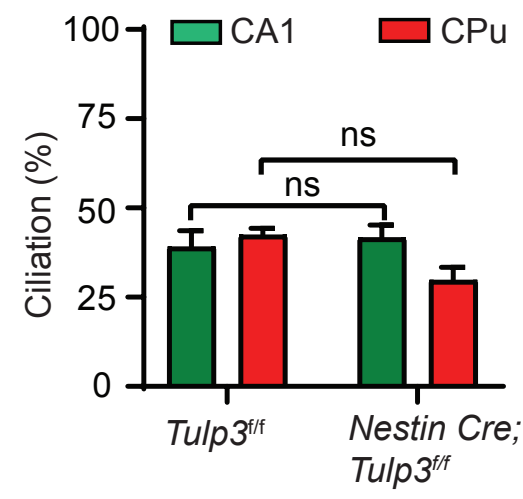

C
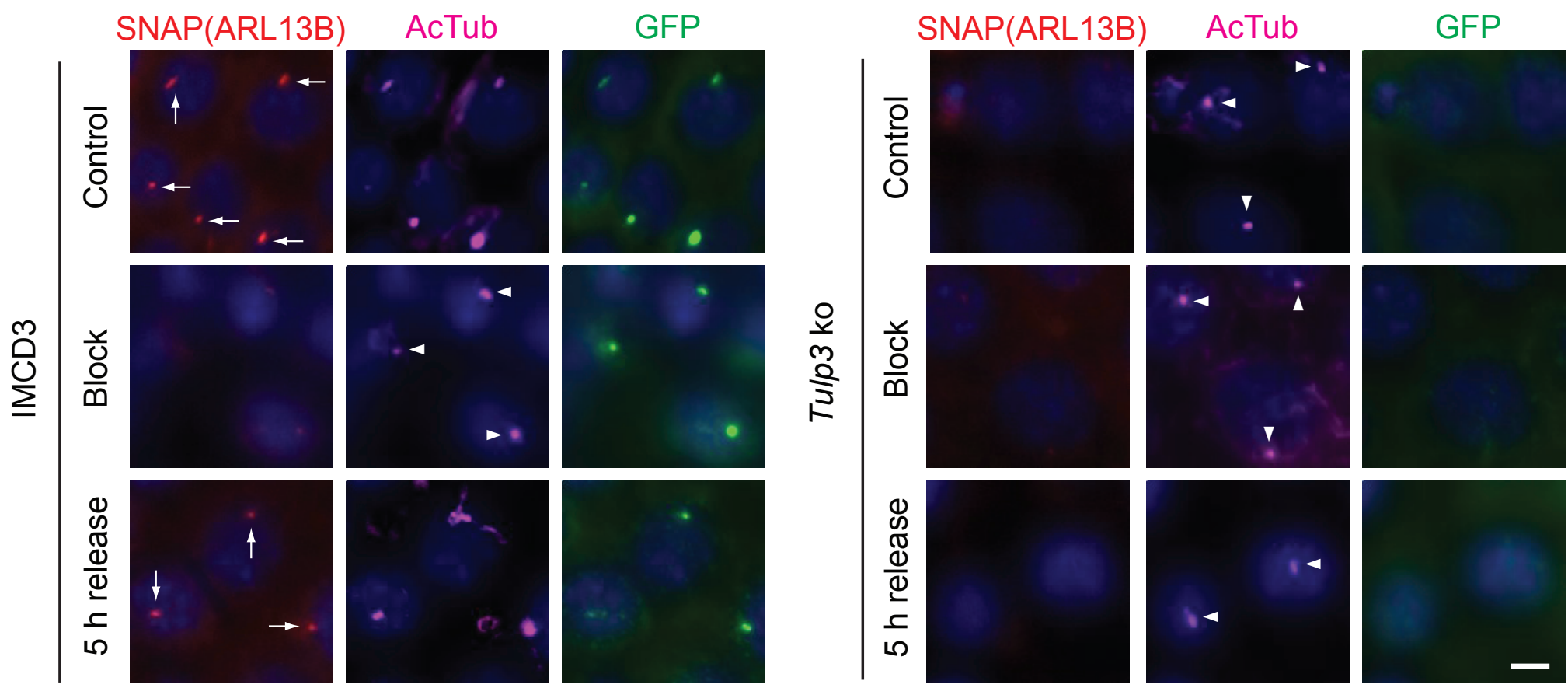

Figure S2 (related to Figure 1). Ciliary localization of Arl13b was unaffected in Nestin-Cre; Tulp ${ }^{\text {fff }}$ brain regions.

(A) Brain sections from CA1 regions in hippocampus from either control or Nestin-Cre; Tulp ${ }^{\text {f/f }}$ mice at P7 were immunostained for Arl13b and AC3 and counterstained for DNA. Quantification shown in Figure $\mathbf{1 H}$.

(B) Cilia (AC3) positive cells were counted from CA1 regions in hippocampus and caudateputamen $(\mathrm{CPu})$ regions of brains from either control or Nestin-Cre; Tulp $3^{f / f}$ mice. Total counted cells were $>200$ from one mouse for control and two mice for Nestin-Cre; Tulp3 $3^{f / f}$. Data represent mean $\pm \mathrm{SD}$.

(C) Representative images for Figure 1J.

Scale, $5 \mu \mathrm{m}$. ns, not significant. 


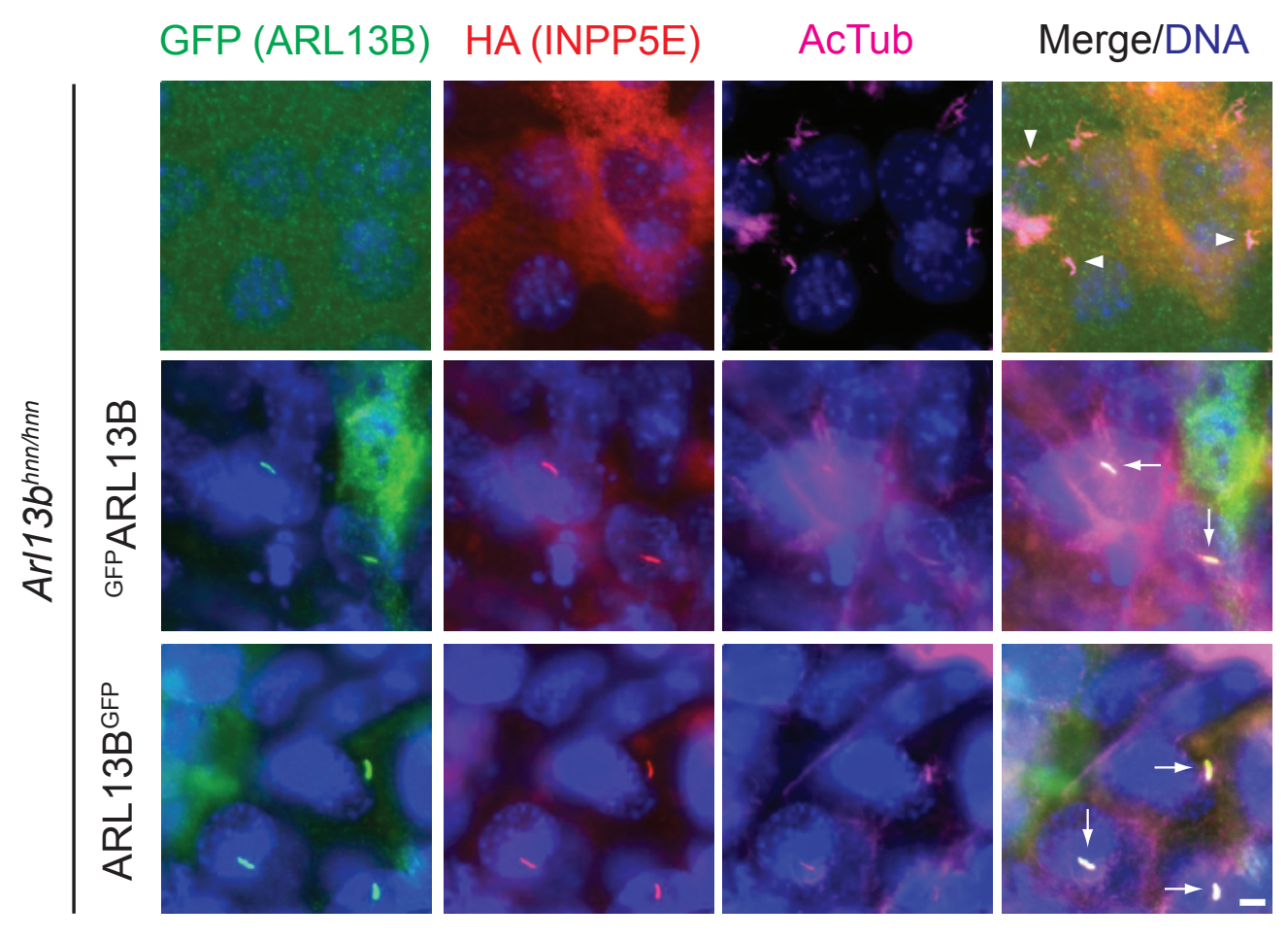

Figure S3 (Related to Figure 2). Tulp3 determines ciliary trafficking of Arl13b dependent cargoes. $\mathrm{N}$ term- or $\mathrm{C}$ term-GFP-Stag (LAP) tagged ARL13B stably expressed in immortalized Arl13 $b^{h n n}$ MEFs along with ${ }^{H A}$ INPP5E were starved for $48 \mathrm{~h}$ and fixed. Fixed cells were immunostained for GFP (ARL13B), HA (INPP5E), acetylated tubulin (AcTub) and counterstained for DNA. Both N- or C-tagged ARL13B fusions trafficked to cilia in Arl13 $b^{\text {hnn }}$ background. ${ }^{H A}$ INPP5E levels in cilia were also restored by either fusion, suggesting rescue from their stable expression.

Scale, $5 \mu \mathrm{m}$. 


\begin{tabular}{|c|c|c|c|c|c|c|}
\hline \multirow[b]{2}{*}{ Peptides with biotin modified lysine } & \multicolumn{6}{|c|}{ Max Intensity (m/z) } \\
\hline & \begin{tabular}{|c|} 
CD8 \\
Gpr161 IC3 \\
WT + LAP \\
TULP3 \\
\end{tabular} & \begin{tabular}{|c|} 
CD8 \\
Gpr161 IC3 \\
5A + LAP \\
TULP3 \\
\end{tabular} & $\begin{array}{c}\text { Fold } \\
\text { change } \\
\text { (WT/5A) }\end{array}$ & $\begin{array}{l}\text { CD8 MCHR1 } \\
\text { IC3 WT + } \\
\text { LAP TULP3 }\end{array}$ & $\begin{array}{l}\text { CD8 MCHR1 } \\
\text { IC3 5A + } \\
\text { LAP TULP3 }\end{array}$ & $\begin{array}{c}\text { Fold } \\
\text { change } \\
(\mathrm{WT/5A})\end{array}$ \\
\hline${ }^{318}{ }^{R K}{ }^{\star M}$ MSVI I PGMTLNHKQI PYQPQNNHDSLLSRW ${ }^{349}$ & $2.13 \mathrm{E}+05$ & $3.46 \mathrm{E}+04$ & 6.16 & ND & ND & \\
\hline${ }^{318}{ }^{R K M S V I}$ I PGMTLNHK * $Q$ I PYQPQNNHDSLLSRW ${ }^{349}$ & $4.73 E+05$ & $1.62 \mathrm{E}+05$ & 2.91 & $3.57 \mathrm{E}+05$ & $1.13 E+04$ & 31.44 \\
\hline${ }^{353}$ RTMENLVELHNK *APVWNSDTQSYVLNFRG ${ }^{381}$ & $3.86 \mathrm{E}+03$ & ND & & ND & ND & \\
\hline 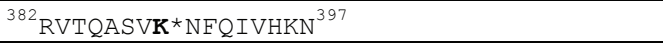 & $3.82 \mathrm{E}+04$ & $2.82 \mathrm{E}+04$ & 1.35 & ND & ND & \\
\hline${ }^{408}{ }^{R V A D D V F T L D Y N Y P L C A V Q A F G I G L S S F D S K}{ }^{\star}{ }^{2} \mathrm{LCE}^{442}$ & $2.47 \mathrm{E}+04$ & ND & & ND & ND & \\
\hline \multicolumn{7}{|l|}{ Unmodified peptides } \\
\hline${ }^{44}$ LEPFMVQPNPEAR $^{56}$ & $7.40 \mathrm{E}+07$ & $3.85 \mathrm{E}+07$ & 1.92 & $9.51 \mathrm{E}+07$ & $6.44 \mathrm{E}+07$ & 1.48 \\
\hline${ }^{232}$ IFLLAAR $^{239}$ & $8.95 \mathrm{E}+07$ & $5.22 \mathrm{E}+07$ & 1.71 & $7.32 \mathrm{E}+07$ & $3.43 E+07$ & 2.14 \\
\hline${ }^{278}$ FTVYDR $^{284}$ & $2.25 \mathrm{E}+08$ & $1.28 \mathrm{E}+08$ & 1.76 & $7.65 \mathrm{E}+07$ & $4.25 \mathrm{E}+07$ & 1.80 \\
\hline
\end{tabular}

$K^{\star}$ denotes biotin modified lysine

$\mathrm{M}$ denotes methionine oxidation across different peptides with same amino acid sequence

MEASRCRLSPSGDSVFHEEMMKMRQAKLDYQRLLLEKRQRKKRLEPFMVQPNPEARLRRAKPRASDEQT PLVNCH 1 TPHSNVILHGIDGPAAVLKPDEVHAPSVSSSVVEEDAENTVDTASKPGLQERLQKHDISESVNFDEETDGISQSA 150 CLERPNSASSQNSTDTGTSGSATAAQPADNLLGDIDYLEDFVYSPAPQGVTVRCRIIRDKRGMDRGLFPTYYMYL 183

EKEENQKI FLLAARKRKKSKTANYLISIDPVDLSREGE SYVGKLRSNLMGTKFTVYDRGICPMKGRGLVGAAHTR

QELAAISYETNVLGFKGPRKMSVIIPGMTLNHRQIPYQPQNNHDSLISRWQNRTMENLVELHNKAPVWNSDTQSY

VLNF'RGRVTQASVKKNFOIVHKND PDYIVMQF'GRVADDVFTLDYNYPLCAVQAFGIGLSSF'DSLLACE

B

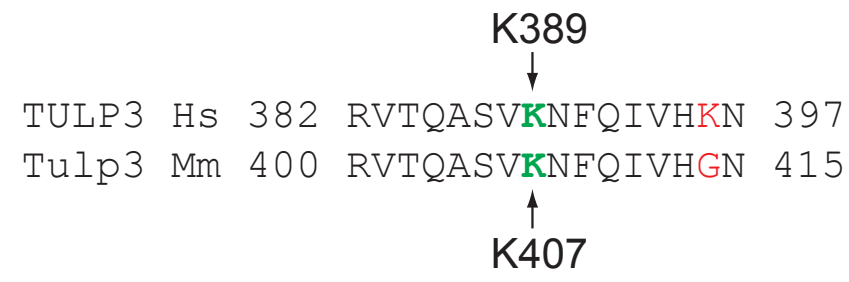

Figure S4 (Related to Figure 6): Lysine K389 in TULP3 is required for interaction with its cargoes.

(A) Sites in TULP3 biotinylated by ciliary localization sequences of GPCRs Gpr161 and MCHR1 in comparison to corresponding mutated CLSs that are non-ciliary (Figure S4A) (Badgandi et al., 2017) identified by mass spectrometry (see Methods).

(B) Alignment of tubby region of TULP3 flanking the Lysine K389 in human TULP3 (Hs, Homo sapiens NP_003315.2) with that from mouse (Ms, Mus musculus NP_035787.1). 
Figure S5 (Related to Figure 8): Arl13b domains required for ciliary localization.

(A) Immortalized Arl13b $b^{V 358 / V 35 A}$ MEFs were starved for $48 \mathrm{~h}$ and immunostained for Arl13b, acetylated tubulin (AcTub) and counterstained for DNA.

(B) T-Rex 293 cells were co-transfected with BirA* tagged TULP3 along with LAP tagged ARL13B or V359A mutant and processed as shown in Figure 5A. Mean \pm SD values indicate Biotin/Stag ratios normalized to CD8 inker control. "n" indicates the number of experiments performed

(C) Bacterially purified GSTTULP3 protein bound to glutathione Sepharose beads was tested for binding with in vitro translated (IVT) Myc full length ARL13B and V359A mutant as in Figure $8 \mathrm{~B}$.

(D) NIH 3T3 wildtype or Tulp3 ko cells were transfected with IA-GFP or I-GFP (with deleted ARL13B C-terminus from IA-GFP) and starved for $48 \mathrm{~h}$. Fixed cells were immunostained for GFP and AcTub.

(E) $A r l 13 b^{h n n}$ cells stably expressing C-LAP tagged D5 were serum starved for $48 \mathrm{~h}$ before fixing and immunostained for GFP, acetylated tubulin (AcTub) and counterstained for DNA.

(F) Cilia lengths of cell lines as described in 8(E). Only ciliated cells in the Arl13b $b^{h n n}$ background stably expressing HA-tagged INPP5E were counted. Arl $13 b^{h n n}$ cells are about $\sim 20 \%$ ciliated (Larkins et al., 2011).

Scale, $5 \mu \mathrm{m}$. ${ }^{* * * *}, \mathrm{p}<0.0001 ;{ }^{* *}, \mathrm{p}<0.001 ;{ }^{* *}, \mathrm{p}<0.01$; ns, not significant. Arrows indicate cilia positive for the indicated proteins while arrowheads indicate negative cilia. 
A

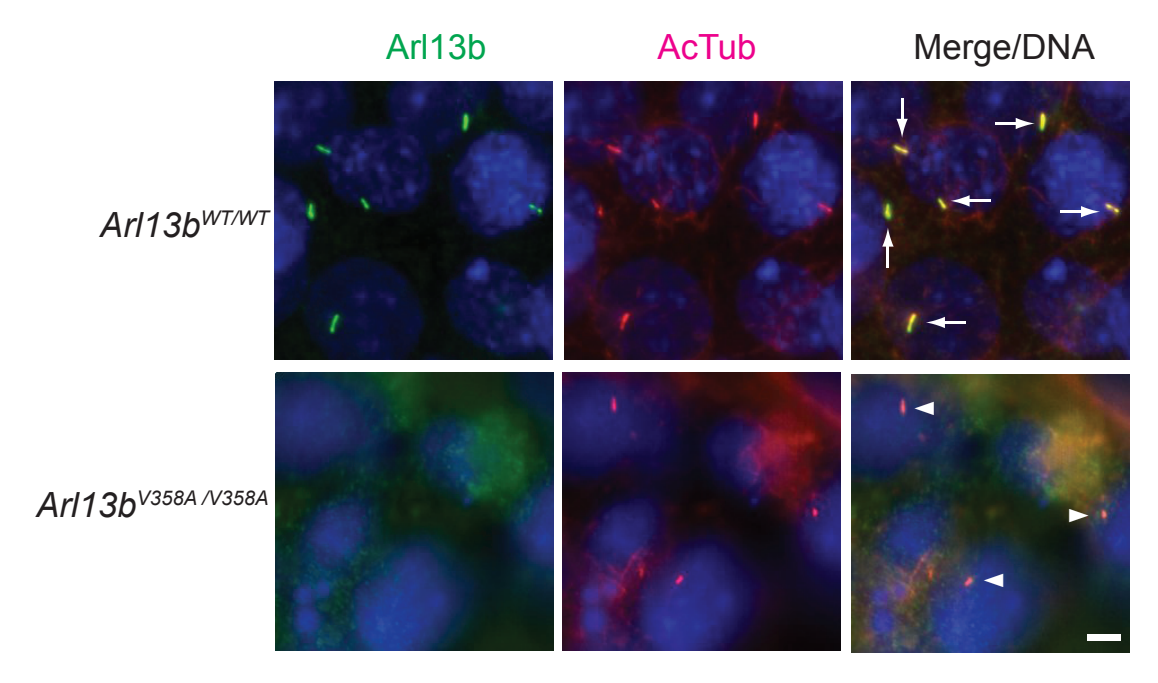

C

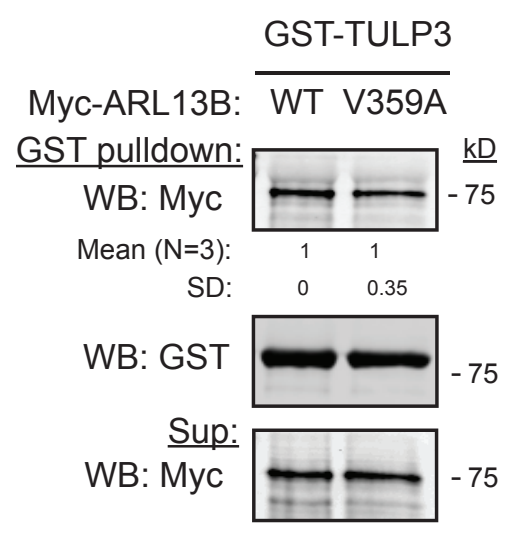

D
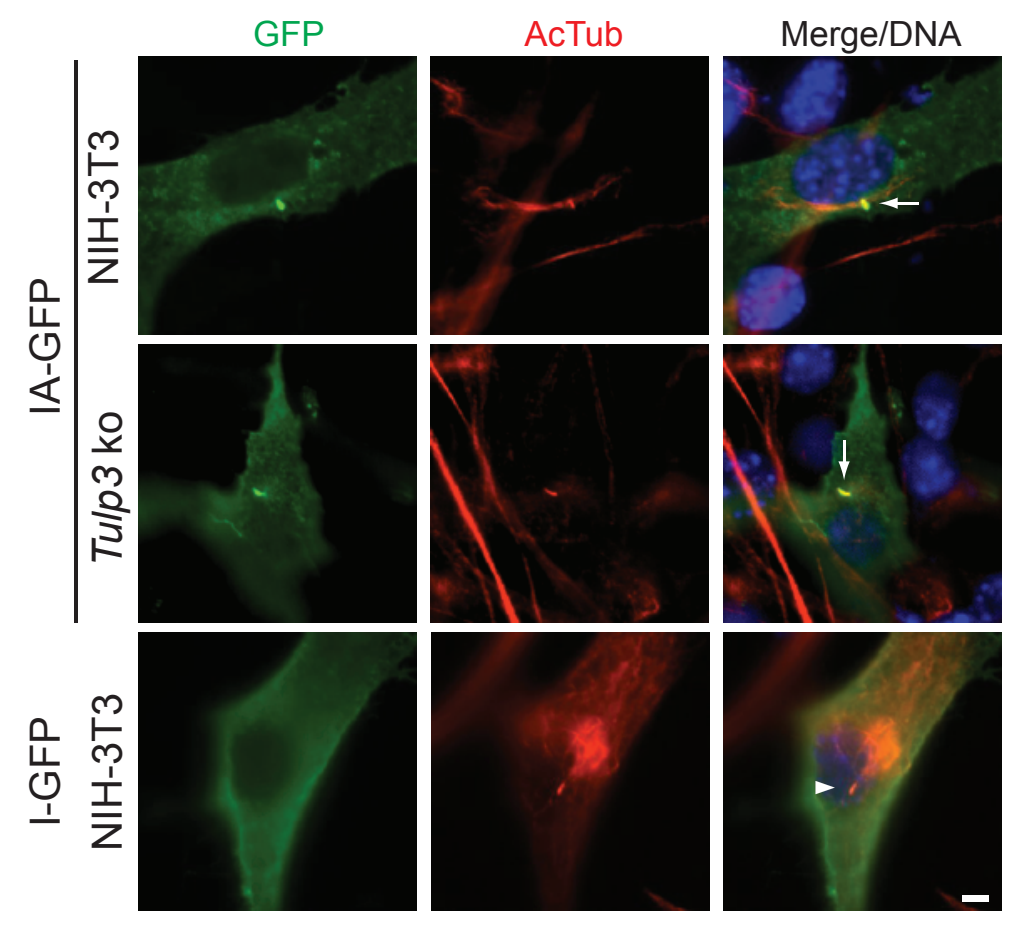

B

BirA*-FlagTULP3

\begin{tabular}{lll}
\cline { 2 - 2 } GFP-TEV- & CD8 \\
STAG: & Linker & ARL13B \\
STt & ARL13B
\end{tabular}
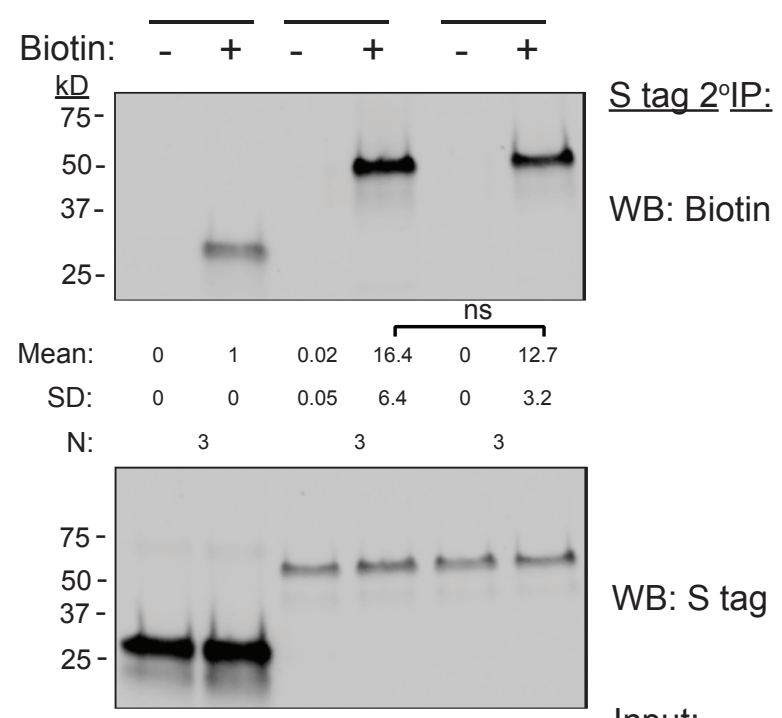

WB: S tag

Input:

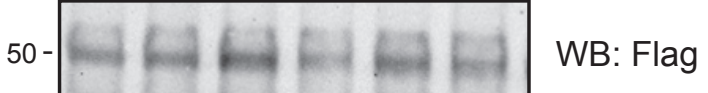

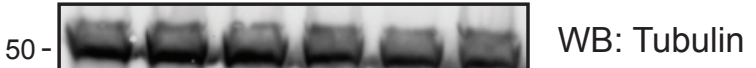

E

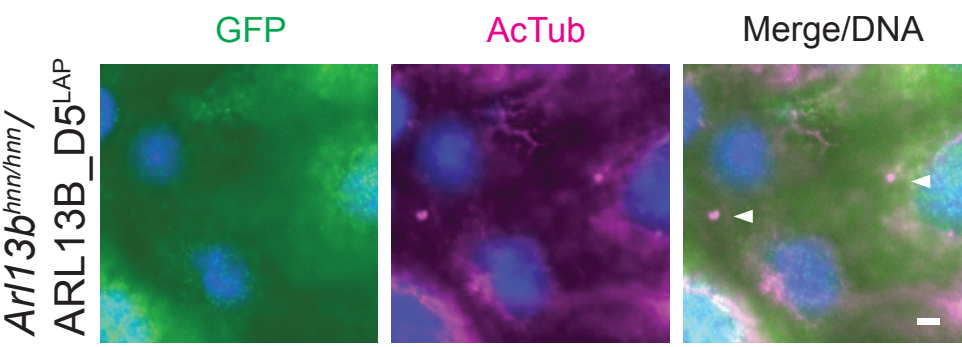

$\mathrm{F}$

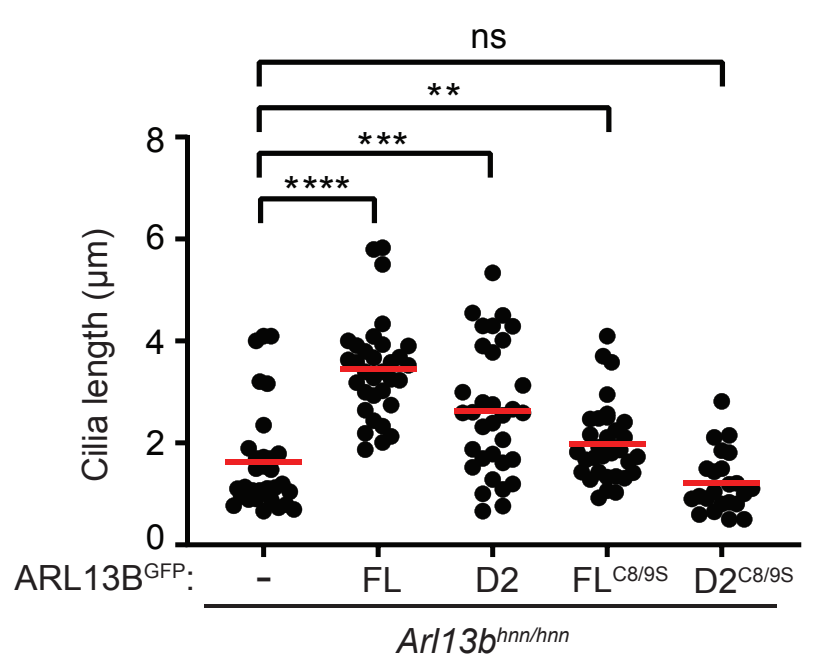




\section{Supplementary Text}

Different reports on ciliary targeting sequences in ARL13B report contrasting results. Here we describe some of these results in chronological order.

First, by transfecting N-terminal (up to GTPase domain) or C-terminal fragment (from CC domain) in wild type mammalian cells, it was shown that neither construct localized to cilia (Hori et al., 2008). The N-terminal region (up to GTPase domain) also showed self-association. Please note that the $\mathrm{N}$-terminal fragment that we found to be ciliary included the $\mathrm{CC}$ domain (Figure 8A, D2). Arl13b mutants lacking CC domain (18-202) in Chlamydomonas completely reduced Arl3GEF activity (Gotthardt et al., 2015) and could prevent access to the amphipathic helix.

Second, by injecting Arl13b mRNA into 1-4 cell stage zebrafish embryos, multiple regions were shown to be necessary for ciliary localization (Duldulao et al., 2009). These included part of the GTPase domain, the coiled coil motif following the GTPase domain and the C-terminus of the protein starting from the coiled coil domain. Many of the ciliary localization and rescue experiments were carried out in a knockout background. Most importantly, a D2 like construct (1307 aa that included $\mathrm{N}$-terminus beyond the $\mathrm{CC}$ domain) was found to be rescuing body curvature and cyst formation despite not being ciliary in localization. We find the D2 construct to be rescuing ${ }^{H A}$ INPP5E levels in cilia but also cilia localized, but only partially rescuing cilia lengths in $A r 113 b^{\text {hnn }}$ MEFs compared to wild type (Figure 8A, E).

Third, initial reports in C. elegans suggested a role of ARL13B palmitoylation in redistributing the protein to the cell body of phasmid neurons, but proximal ciliary localization persisted (Cevik et al., 2010; Cevik et al., 2013). Deleting the C-terminus RVxP motif (RVVP sequence in C. elegans) also did not prevent proximal ciliary localization of ARL13B in phasmid neurons, rather caused it to distribute more distally in addition to forming cell body aggregates (Cevik et al., 2013). These ARL13B fusions were all expressed in presence of wild type protein. Of note, the RVVP motif deleted ARL13B transgene functions as a dominant negative when 
injected in wild type C. elegans for dye-filling (such dye filling defects are also a feature of $A R L 13 B$ null allele) (Cevik et al., 2013). Such dominant negative effects suggest mutual interactions in compromising wild-type protein function. Similar dominant negative effects are also seen in palmitoylation mutants (Cevik et al., 2010), suggesting coupling with membrane to be critical for mutual interactions in compromising wild-type protein function.

Fourth, by transiently transfecting $\mathrm{N}$-terminal (1-212, disrupting CC) and C-terminal Arl13b (210-427) in Arl13 $b^{h n n}$ background, it was shown that neither fragment was ciliary (Larkins et al., 2011). Please note that the N-terminal construct that we found to be ciliary included the CC domain (Figure 8A, D2). A single amino acid mutation in mouse RVxP motif, Arl13b $1358 \mathrm{~A}$, was shown to be nonciliary upon transient transfection in $A r l 13 b^{h n n}$ MEFs (Mariani et al., 2016). Palmitoylation motif mutants $\left(A r l 13 b^{C 8 / 9 S}\right)$ were also found to be partially ciliary ( $20 \%$ compared to $\sim 80 \%$ localization for wild type) when transfected in $A r l 13 b^{h n n}$ background (unlike our results using stable expression that showed robust localization in cilia and ${ }^{\mathrm{HA}}$ INPP5E rescue in cilia) (Figure 8A, 8E). MEFs generated from endogenous Arl13b $b^{V 358 A / 358 A}$ knock-in embryos also showed no steady state levels of the mutant protein in cilia (Gigante et al., 2020) (Figure S5A).

Fifth, another study using transfected tagged $A R L 13 B$ constructs in wild type ARL13B background reported the RVEP-to-AAEA mutation to disrupt ciliary localization in RPE cells, but also form aggregates in the cytoplasm (Nozaki et al., 2017). GTPase domain mutants (T35N, R79Q) were ciliary as reported earlier (Humbert et al., 2012). The INPP5E binding region in ARL13B was pinpointed to both GTPase and CC domains (Nozaki et al., 2017) and GTPase domain mutants (T35N, R79Q) also reduced INPP5E binding (Humbert et al., 2012; Nozaki et al., 2017). Deletion of only GTPase domain (unlike zebrafish experiments (Duldulao et al., 2009)) or swapping an ARF6 GTPase domain still retained ciliary localization (Nozaki et al., 2017). The authors were not able to infer if $\operatorname{ARL} 13 B(\triangle C C)$ localizes to cilia, as this mutant fused to tags formed aggregates in the cytoplasm (Nozaki et al., 2017), and could explain lack of ciliary expression seen in zebrafish experiments (Duldulao et al., 2009). 
KEY RESOURCE TABLE

\begin{tabular}{|c|c|c|}
\hline REAGENT OR RESOURCE & SOURCE & IDENTIFIER \\
\hline \multicolumn{3}{|l|}{ Antibodies and related } \\
\hline $\begin{array}{l}\text { Mouse monoclonal anti-Acetylated } \\
\text { tubulin }\end{array}$ & Sigma & $\begin{array}{l}\text { Cat\# T6793 } \\
\text { RRID: AB_477585 }\end{array}$ \\
\hline Rabbit polyclonal anti-Arl13b (IF) & $\begin{array}{l}\text { Tamara Caspary } \\
\text { (Emory University) }\end{array}$ & \\
\hline Rabbit polyclonal anti-Arl13b (WB) & Proteintech & $\begin{array}{l}\text { Cat\# 17711-1-AP } \\
\text { RRID: AB 2060867 }\end{array}$ \\
\hline Mouse monoclonal anti-Arl13b (IF) & NeuroMab Facility & $\begin{array}{l}\text { Cat\# N295B/66 } \\
\text { RRID: AB_2750771 }\end{array}$ \\
\hline Chicken anti-GFP (IF) & Abcam & $\begin{array}{l}\text { Cat\# ab13970 } \\
\text { RRID: AB } 300798\end{array}$ \\
\hline Rabbit polyclonal anti-Smo & $\begin{array}{l}\text { Kathryn Anderson } \\
\text { (MSKCC) }\end{array}$ & $\begin{array}{l}\text { (Ocbina and Anderson, } \\
\text { 2008) }\end{array}$ \\
\hline Rabbit polyclonal anti-Tulp3 & $\begin{array}{l}\text { Peter Jackson } \\
\text { (Stanford University) }\end{array}$ & $\begin{array}{l}\text { (Mukhopadhyay et al. } \\
\text { 2010) }\end{array}$ \\
\hline Rabbit polyclonal anti-Gpr161 & Custom-made & (Pal et al. 2016) \\
\hline Rabbit polyclonal anti-IFT139 & $\begin{array}{l}\text { David Beier (Seattle } \\
\text { Children's) }\end{array}$ & (Tran et al., 2008) \\
\hline Rabbit polyclonal anti-GFP (WB) & Abcam & $\begin{array}{l}\text { Cat\# ab290 } \\
\text { RRID: AB } 303395\end{array}$ \\
\hline Mouse monoclonal anti-S tag & EMD Millipore & Cat\# MAC112 \\
\hline Goat polyclonal anti-Myc tag & Abcam & $\begin{array}{l}\text { Cat\# ab9132 } \\
\text { RRID: AB } 307033\end{array}$ \\
\hline Rat monoclonal anti-HA & Roche & $\begin{array}{l}\text { Cat\# 11867423001 } \\
\text { RRID: AB } 390918\end{array}$ \\
\hline Goat polyclonal anti-DDDDK (Flag) tag & Abcam & $\begin{array}{l}\text { Cat\# ab1257 } \\
\text { RRID: AB } 299216\end{array}$ \\
\hline Mouse monoclonal anti-MBP & NEB & Cat\# E8032S \\
\hline Mouse monoclonal anti-GST & Sigma & $\begin{array}{l}\text { Cat\# G1160 } \\
\text { RRID: AB } 259845\end{array}$ \\
\hline Rabbit polyclonal anti-ADCY3 & LSBio & Cat\# LS-C204505 \\
\hline Rabbit polyclonal anti-Aqp2 & Sigma & $\begin{array}{l}\text { Cat\# A7310 } \\
\text { RRID: AB } 476762\end{array}$ \\
\hline Mouse monoclonal anti-Aqp2 & Santa Cruz & $\begin{array}{l}\text { Cat\# sc-515770 } \\
\text { RRID: AB } 2810957\end{array}$ \\
\hline Rabbit polyclonal anti-INPP5E & Proteintech & $\begin{array}{l}\text { Cat\# 17797-1-AP } \\
\text { RRID: AB 2167120 }\end{array}$ \\
\hline Rabbit polyclonal anti-NPHP3 & Proteintech & $\begin{array}{l}\text { Cat\# 22026-1-AP } \\
\text { RRID: AB 2878975 }\end{array}$ \\
\hline Rabbit Monoclonal anti-LKB1 & CST & Cat\# 13031T \\
\hline hFAB Rhodamine anti-Tubulin & Bio-Rad & $\begin{array}{l}\text { Cat\# } 12004165 \\
\text { RRID: AB } 2884950\end{array}$ \\
\hline IRDye 680 Streptavidin & LI-COR & Cat\# 926-68079 \\
\hline $\begin{array}{l}\text { Alexa Fluor 488-, 594-, 647- conjugated } \\
\text { secondary antibodies }\end{array}$ & Life Technologies & \\
\hline IRDye tagged secondary antibodies & LI-COR & \\
\hline Hoechst 33342 & Life Technologies & H3570 \\
\hline
\end{tabular}




\begin{tabular}{|c|c|c|}
\hline BG-Block & NEB & S9106 \\
\hline TMR Star & NEB & S9105 \\
\hline \multicolumn{3}{|l|}{ Chemicals, Media, and Kits } \\
\hline Penicillin/Streptomycin & Sigma & P4333 \\
\hline Glutamine & Sigma & G7513 \\
\hline Polyfect & Qiagen & 301105 \\
\hline PEI-Max & Polysciences & $24765-2$ \\
\hline DMEM-High Glucose & Sigma & D5796 \\
\hline DMEM F12 & Sigma & D6421 \\
\hline Bovine calf serum & Sigma & $12133 \mathrm{C}$ \\
\hline Fetal bovine serum & Sigma & F0926 \\
\hline \multicolumn{3}{|l|}{ Cosmic serum } \\
\hline Paraformaldehyde & $\begin{array}{l}\text { Electron microscopy } \\
\text { solutions }\end{array}$ & 15710 \\
\hline Normal donkey serum & $\begin{array}{l}\text { Jackson Immuno } \\
\text { Research, West } \\
\text { Grove, PA }\end{array}$ & $\begin{array}{l}\text { Cat\# 017-000-121 } \\
\text { RRID: AB_2337258 }\end{array}$ \\
\hline Fluoromount-G & Southern Biotech & $0100-01$ \\
\hline Permount & $\begin{array}{l}\text { ThermoFisher } \\
\text { Scientific }\end{array}$ & SP15-100 \\
\hline Biotin & Sigma & Cat\# B4639 \\
\hline $\begin{array}{l}\text { GenElute mammalian total RNA } \\
\text { purification kit }\end{array}$ & Sigma & RTN350 \\
\hline DNase I & Sigma & D5307 \\
\hline SYBR Green Quantitative RT-qPCR Kit & Sigma & QR0100 \\
\hline $\begin{array}{l}\text { Kicqstart One-Step Probe RT-qPCR } \\
\text { ReadyMix }\end{array}$ & Sigma & KCQS07 \\
\hline $\begin{array}{l}\text { TNT Sp6 high-yield wheat germ protein } \\
\text { expression system }\end{array}$ & Promega & L3261 \\
\hline Synthetic mounting media & Fisher Chemical & SP15 \\
\hline $\begin{array}{l}\text { Mini-PROTEAN TGX Precast Protein } \\
\text { Gels }\end{array}$ & Bio-Rad & \\
\hline \multicolumn{3}{|l|}{ Experimental Models: Organisms } \\
\hline Mouse: Tulp3 $3^{f / f}$ & MRC, Harwell & (Hwang et al, 2019) \\
\hline Mouse: HoxB7-Cre & $\begin{array}{l}\text { O'Brien Kidney } \\
\text { Research Core of UT } \\
\text { Southwestern }\end{array}$ & \\
\hline Mouse: Ksp-Cre & $\begin{array}{l}\text { O'Brien Kidney } \\
\text { Research Core of UT } \\
\text { Southwestern }\end{array}$ & \\
\hline Mouse: Nestin-Cre & JAX & Stock No. 003771 \\
\hline \multicolumn{3}{|l|}{ Experimental Models: Cell Lines } \\
\hline IMCD3 FIp-In & \begin{tabular}{|l} 
Peter Jackson \\
(Stanford University)
\end{tabular} & (Torres et al, 2009) \\
\hline RPE hTERT & ATCC & ATCC CRL-4000 \\
\hline NIH 3T3 Flp-In & ATCC & R76107 \\
\hline
\end{tabular}




\begin{tabular}{|c|c|c|}
\hline 3T3-L1 & \begin{tabular}{|l|} 
Peter Michaely \\
(UT Southwestern) \\
\end{tabular} & \\
\hline TRex-293 FLP-In & Life Technologies & R78007 \\
\hline \multicolumn{3}{|l|}{ Experimental Models: Primary cells } \\
\hline Tulp3 knockout MEFs & This study & \\
\hline Tulp3 K407I/K407I MEFs & This study & \\
\hline \multicolumn{3}{|l|}{$\begin{array}{l}\text { Experimental Models: Immortalized } \\
\text { MEFs }\end{array}$} \\
\hline Arl13 $b^{h n n}$ MEFs and wild type controls & $\begin{array}{l}\text { Tamara Caspary } \\
\text { (Emory University) }\end{array}$ & (Larkins et al, 2011) \\
\hline Arl13b $b^{V 358 A}$ MEFs and wild type controls & $\begin{array}{l}\text { Tamara Caspary } \\
\text { (Emory University) }\end{array}$ & (Gigante et al, 2020) \\
\hline \multicolumn{3}{|l|}{ Recombinant DNA } \\
\hline pGLAP5 & Addgene & 19706 \\
\hline pG-LAP1 & Addgene & 19702 \\
\hline pENTR223-ARL13B & DNAsu & HsCD00511796 \\
\hline pENTR221-INPP5E & Life Technologies & $\mathrm{IOH} 40212$ \\
\hline NPHP3 (1-203 aa) & GeneArt & Synthesized \\
\hline Gateway PLUS shuttle clone for CYS1 & GeneCopoeia & GC-Y0203-CF \\
\hline Gatewaytized LAP1 or LAP5 in pBABE & This study & \\
\hline pQXIN-Myc-TULP3 & This study & \\
\hline pQXIN-HA-INPP5E & This study & \\
\hline pcDNA5-BirA-FLAG N-DEST & Anne-Claude Gingras & \\
\hline pcDNA5-BirA-FLAG C-DEST & Anne-Claude Gingras & \\
\hline \multicolumn{3}{|l|}{ pCS2-Myc-DEST } \\
\hline pOCC29 & & (Lemaitre et al., 2019) \\
\hline GFP-SNAP-ARL13B in pBABE & This study & \\
\hline \multicolumn{3}{|l|}{ Software and Algorithms } \\
\hline ImageJ software & $\begin{array}{l}\text { National Institutes of } \\
\text { Health, Bethesda, MD }\end{array}$ & \\
\hline GraphPad Prism & $\begin{array}{l}\text { GraphPad, La Jolla, } \\
\text { CA }\end{array}$ & \\
\hline PictureFrame 3.0 acquisition software & $\begin{array}{l}\text { Optronics, Inc. Goleta, } \\
\text { CA, USA }\end{array}$ & \\
\hline Sequest & $\begin{array}{l}\text { Thermo Fisher Scientific, } \\
\text { Waltham, MA }\end{array}$ & \\
\hline \multicolumn{3}{|l|}{ Other } \\
\hline PIP Strip & Thermo Fisher & P23751 \\
\hline $\begin{array}{l}\text { Thermo-Fisher Excelsior Automated } \\
\text { Tissue Processor }\end{array}$ & $\begin{array}{l}\text { ThermoFisher } \\
\text { Scientific }\end{array}$ & A82300001 \\
\hline Paraplast Plus paraffin bath & Leica & 39602004 \\
\hline $\begin{array}{l}\text { Thermo-Shandon Histocenter } 2 \\
\text { Embedding Workstation }\end{array}$ & $\begin{array}{l}\text { ThermoFisher } \\
\text { Scientific }\end{array}$ & 6400012D \\
\hline $\begin{array}{l}\text { Leica stereomicroscope (M165 C) with } \\
\text { digital camera (DFC500) }\end{array}$ & Leica & \\
\hline
\end{tabular}


bioRxiv preprint doi: https://doi.org/10.1101/2021.05.25.445488; this version posted May 25, 2021. The copyright holder for this preprint (which was not certified by peer review) is the author/funder. All rights reserved. No reuse allowed without permission.

\begin{tabular}{|l|l|l|}
\hline Zeiss stereomicroscope (Discovery.V12) & Zeiss & \\
\hline Zeiss LSM780 confocal microscope & Zeiss & \\
\hline Leica DM2000 photomicroscope & Leica & DRS-601 \\
\hline Sakura DRS-601 x-y-Z robotic-stainer & $\begin{array}{l}\text { Sakura-FineTek, } \\
\text { Torrance, CA }\end{array}$ & \\
\hline CFX96 thermocycler & Bio-Rad & $12-550-15$ \\
\hline Superfrost ${ }^{\circledR}$ Plus slides & Fisher Scientific & \\
\hline Vitros 250 chemistry analyzer & GMI Inc., Ramsey, MN & \\
\hline
\end{tabular}

